Aus der Abteilung Allgemeinmedizin

(Prof. Dr. med. M. M. Kochen, MPH, FRCGP)

im Zentrum Innere Medizin

der Medizinischen Fakultät der Universität Göttingen

\title{
Arzt des Vertrauens: \\ Präferenzen schizophrener Patienten \\ für ihre haus- und fachärztliche Betreuung
}

\author{
INAUGURAL - DISSERTATION \\ zur Erlangung des Doktorgrades \\ der Medizinischen Fakultät \\ der Georg-August-Universität zu Göttingen \\ vorgelegt von \\ Kirstin Kühmel \\ aus \\ Eberswalde
}

Göttingen 2007 
I. Berichterstatter: Prof. Dr. disc. pol. W. Himmel

II. Berichterstatter/in:

III. Berichterstatterlin:

Tag der mündlichen Prüfung: 


\section{Inhaltsverzeichnis}

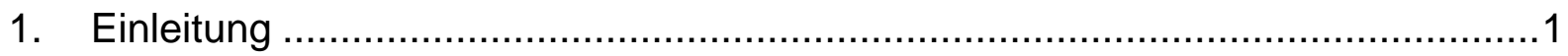

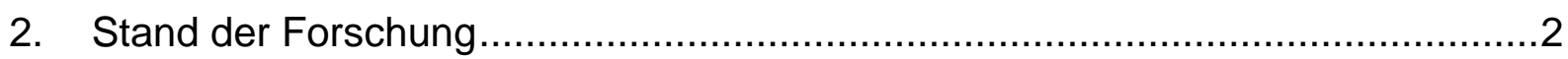

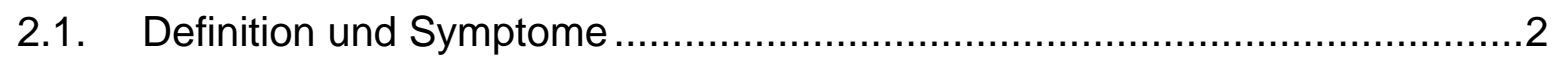

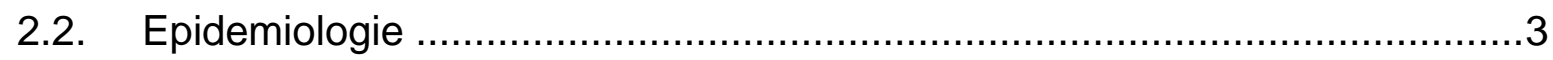

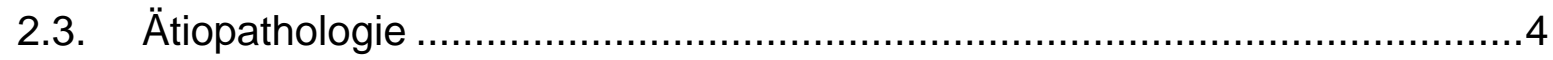

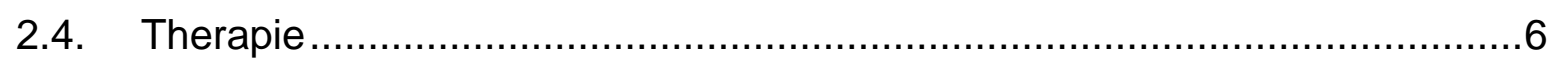

2.5. Die ambulante Behandlung schizophrener Patienten ...............................

2.6. Kriterien der Arztwahl und Arztbewertung psychisch Kranker.....................9

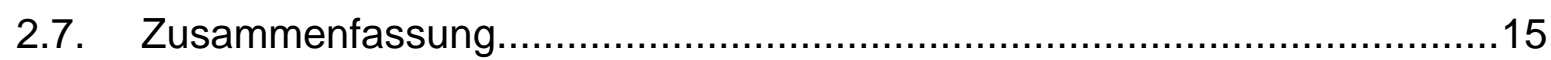

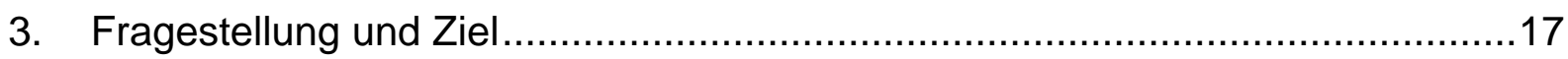

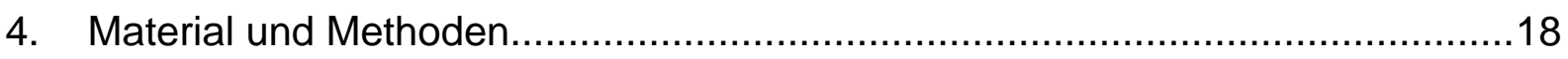

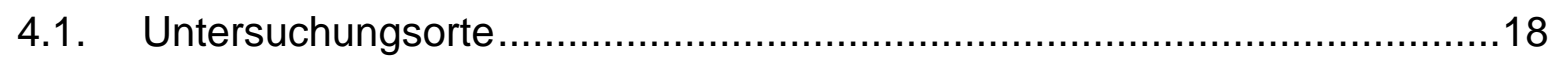

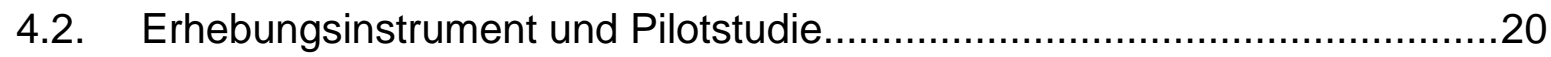

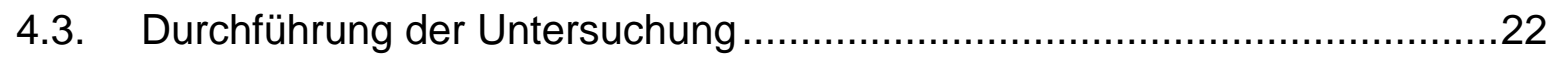

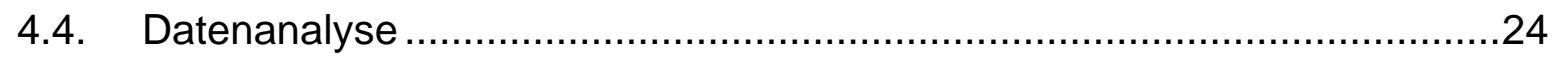

4.5. Datenschutz und Genehmigung durch die Ethik-Kommission ..................24

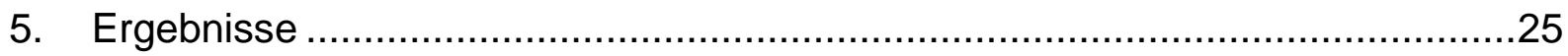

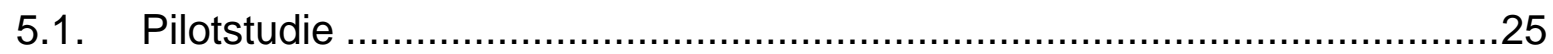

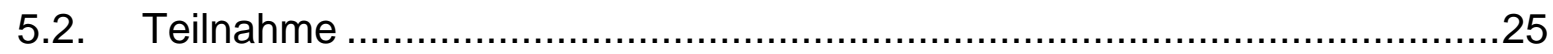

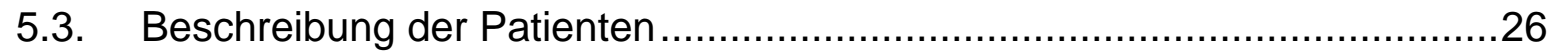

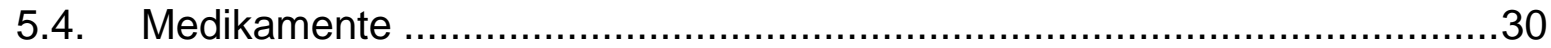

5.5. Diagnosen

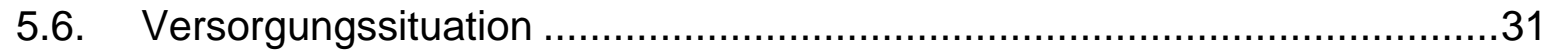

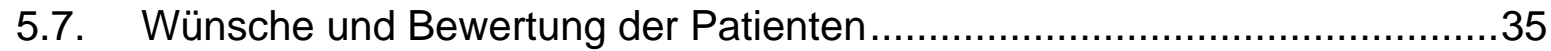

5.8. Bewertung der Arztgruppen und Schulbildung der Befragten ..................42

5.9. Bewertung der Arztgruppen und Rekrutierungsort ................................43 
5.10. Bewertung der Arztgruppen und Geschlecht...............................44

5.11. Rangliste der Patientenerwartung an beide Arztgruppen ...................45

5.12. Merkmale mit höchster Patientenbewertung ..................................46

5.13. Persönliche Wünsche der Patienten an die Ärzte............................48

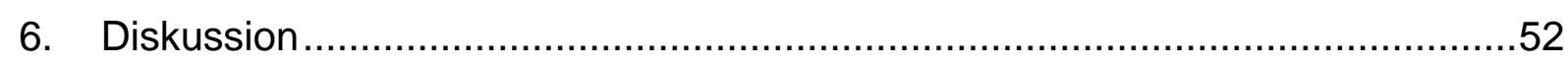

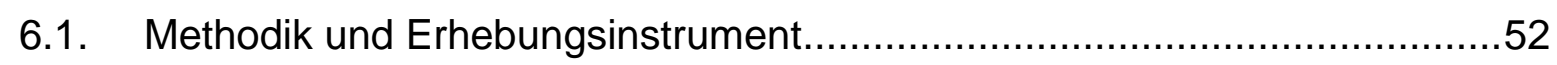

6.2. Untersuchungsorte und Rücklaufquote .......................................5

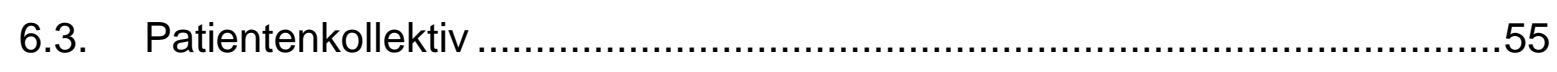

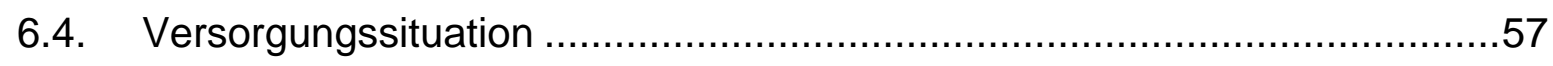

6.5. Wünsche der Patienten an die Arztgruppen..................................63

6.6. Bewertung der Arztgruppen durch die Patienten ................................67

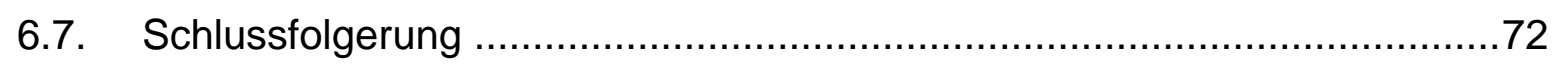

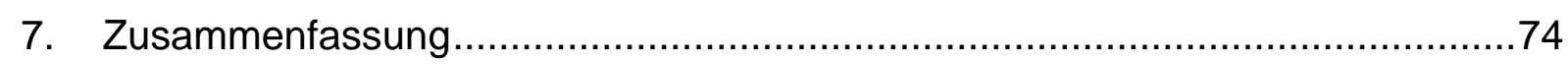

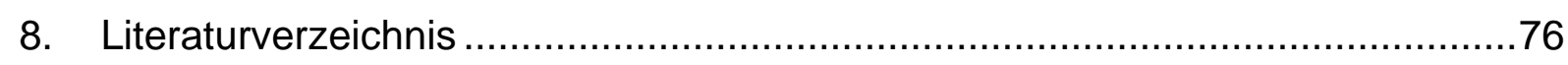

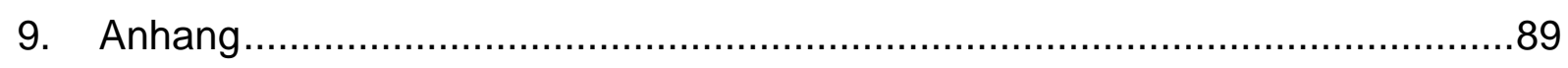

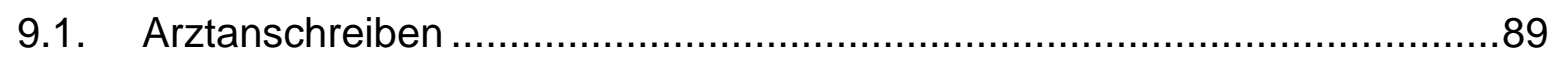

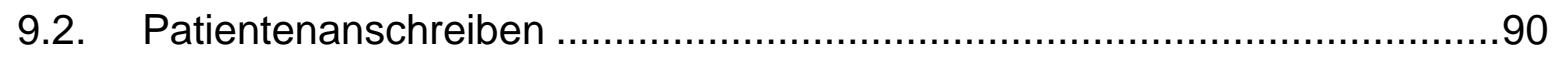

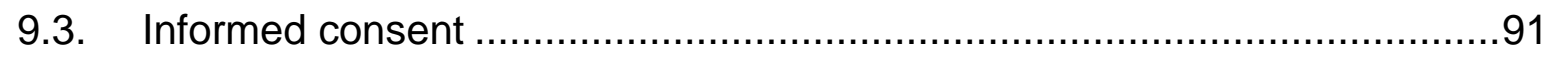

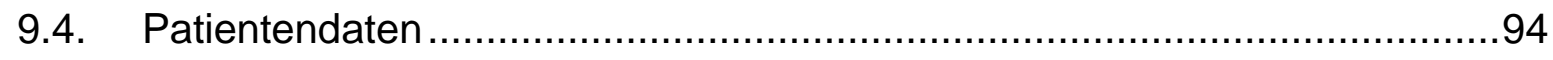

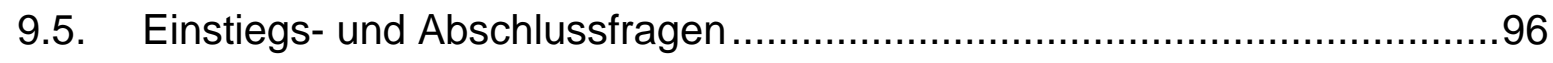

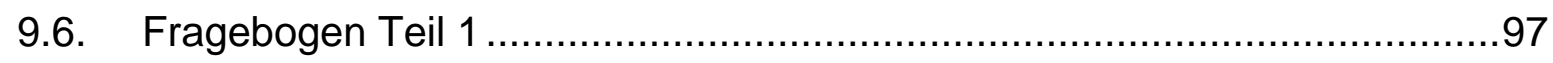

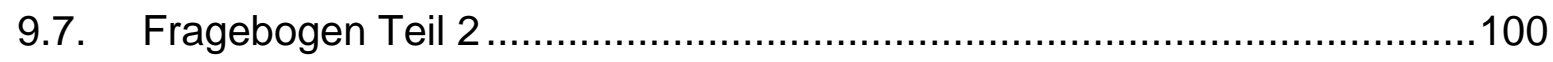

9.8. Grundauszählung Patienten (Gesamtstichprobe N=111)...................103 


\section{Tabellenverzeichnis:}

Tabelle 1: Symptome zur Diagnosesicherung der Schizophrenie nach ICD-10 .........3

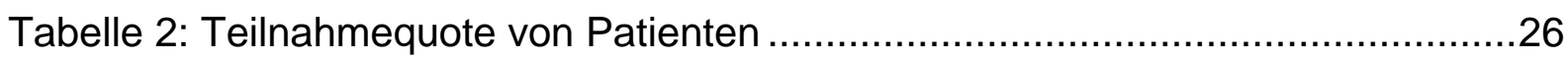

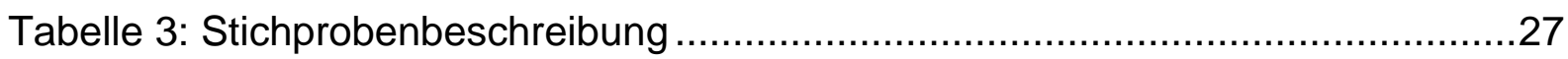

Tabelle 4: Verordnungshäufigkeit oraler Medikamente .................................30

Tabelle 5: Selbstgenannte Diagnosen der Patienten .................................... 31

Tabelle 6: Gründe für die Konsultation des Hausarztes bzw. des Nervenarztes .......32

Tabelle 7: Bedeutung der Praxisorganisation - Vergleich der Praxistypen ...............35

Tabelle 8: Bewertung der Praxisorganisation - Vergleich der Praxistypen ...............36

Tabelle 9: Bedeutung der Fachkompetenz - Vergleich der Praxistypen .................37

Tabelle 10: Bewertung Fachkompetenz - Vergleich der Praxistypen ......................38

Tabelle 11: Bedeutung der Arzt-Patienten-Beziehung - Vergleich der Praxistypen..39

Tabelle 12: Bewertung der Arzt-Patienten-Beziehung - Vergleich der Praxistypen ..40

Tabelle 13: Bedeutung der Beratung und Information für die Patienten Vergleich der Praxistypen

Tabelle 14: Bewertung der Beratung und Information - im Vergleich der Praxistypen .....

Tabelle 15: Bewertung der Arztgruppen und Bildungsstand der Patienten

Tabelle 16: Bewertung der Arztgruppen und Rekrutierungsort der Patienten 44

Tabelle 17: Bewertung der Arztgruppen und Geschlecht der Patienten. .45

Tabelle 18: Die 10 wichtigsten Merkmale* des Hausarztes aus Sicht der Patienten.45

Tabelle 19: Die 10 wichtigsten Merkmale* des Nervenarztes aus Sicht der Patienten

Tabelle 20: Merkmale* des Hausarztes mit hoher Bewertung ...........................47

Tabelle 21: Merkmale* des Facharztes mit hoher Bewertung ............................48

Tabelle 22: Gewünschte Fähigkeiten des Arztes .......................................49

Tabelle 23: Gewünschte Merkmale der Arzt-Patienten-Beziehung ......................50

Tabelle 24: Gewünschte Rahmenbedingungen und Inhalte der Konsultation ..........51 


\section{Abbildungsverzeichnis:}

Abbildung 1: Alter zu Beginn der psychischen Erkrankung .............................28

Abbildung 2: Erste psychiatrische Behandlung …...................................... 29

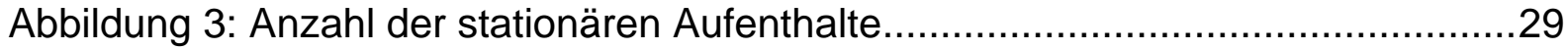

Abbildung 4: Hauptansprechpartner der Patienten für psychische Probleme und Medikamente 32

Anmerkung:

Der besseren Lesbarkeit wegen wird im Text die männliche Sprachform gewählt. Selbstverständlich sind beide Geschlechter gemeint.

Der Begriff „Hausarzt“ fasst praktische Ärzte, Fachärzte für Allgemeinmedizin und hausärztlich tätige Internisten zusammen. Mit dem Begriff „Facharzt“ bzw. „Spezialist" sind Psychiater und Neurologen gemeint. 


\title{
1. Einleitung
}

\author{
„schizophrenie“ \\ „du weißt dich als einen kranken verworfnen, \\ wenn dir begegnend der nachbar \\ nicht mehr grüßt, befremden und furcht im gesicht, \\ wenn deine mutter, ihre hände über deiner \\ rente haltend, dir wortlos zehn mark zuschiebt, \\ wissend, du besorgst dir dafür pornographie, \\ wenn sich am telefon die alten freunde \\ von ihren frauen verleugnen lassen \\ und du auf briefe keine antwort mehr erhältst, \\ wenn du dich vor dir selbst entsetzt, \\ im spiegel erblickend ein verzerrtes gesicht, \\ in dem ein stummes verzagen sich ausschreit."
}

Reinhart Meinel

(aus Häfner 2001, S.171)

Schizophrenie ist ein Wort, das auch im 21. Jahrhundert noch mit vielen Vorurteilen belastet ist. Gründe hierfür sind in der Vergangenheit zu suchen. Lange Zeit wusste man fast nichts über die Ursachen der Krankheit und ihre Behandlung. Auch heute dürfte das Wissen in der Bevölkerung über die Krankheit eher gering sein, und allzu oft werden Patienten mit Schizophrenie ausgegrenzt und geraten in eine soziale Isolation. Bedingt durch die Symptomatik der Krankheit, neigen die Patienten zum sozialen Rückzug und verfügen selten über ein stabiles Netz, das sie in Krisensituationen auffangen könnte. Allein deshalb ist es wünschenswert, dass die behandelnden Ärzte - im ambulanten Bereich vor allem Neurologen, Psychiater und Hausärzte die Bedürfnisse ihrer Patienten kennen und achten, um sie in schwierigen Situationen unterstützen zu können und ihnen als kompetenter und einfühlsamer Begleiter zur Seite zu stehen. Was schizophrene Patienten von der ambulanten Betreuung erwarten, welchem Arzt sie bezüglich der Behandlung ihrer seelischen Erkrankung Vertrauen schenken und wie sie ihre Versorgungssituation beurteilen, ist Thema dieser Arbeit. 


\section{Stand der Forschung}

\subsection{Definition und Symptome}

Der Begriff Schizophrenie bedeutet Spaltungsirresein. Es handelt sich dabei um eine Psychose mit Verlust des Strukturzusammenhangs der Persönlichkeit und Spaltung von Denken, Affekt und Erleben (Bogensberger et al. 1993). Seit über 100 Jahren werden Versuche unternommen, Psychosen aus dem schizophrenen Formenkreis, die heute mit der Diagnose F 20.0-6 nach ICD 10 (WHO 2006) zusammengefasst werden, von anderen psychischen Krankheiten abzugrenzen.

Kraepelin, der 1896 den Begriff „dementia praecox“ prägte, ging davon aus, dass dieser Erkrankung ein degenerativer hirnorganischer Prozess zugrunde liegt, der schließlich zur Demenz führt (Längle und Mayenberger 2000). Diese Ansichtsweise wurde jedoch 1911 von Bleuler widerlegt:

„Leider konnten wir uns der unangenehmen Aufgabe nicht entziehen, einen neuen Namen für die Krankheitsgruppe zu schmieden. Der bisherige ist zu unhandlich. Man kann damit nur die Krankheit benennen, nicht aber die Kranken (...). So blieb mir nichts anderes übrig, als hier die Krankheit mit einem Namen zu bezeichnen, der weniger missverständlich ist.(...) Ich nenne die Dementia praecox Schizophrenie, weil ich zu zeigen hoffe, die Spaltung der verschiedensten psychischen Funktionen eine ihrer wichtigsten Eigenschaften ist" (Bleuler 1911, S. 4,5).

Bleuler führte den Begriff der Schizophrenie ein und verstand darunter die Spaltung der verschiedensten psychischen Funktionen (Längle und Mayenberger 2000). Nach heutiger Auffassung und gemäß ICD-10 sind für die Diagnose „Schizophrenie“ mindestens ein oder mehrere Symptome erforderlich: und zwar mindestens ein eindeutiges Symptom aus den in Tabelle 1 genannten Gruppen 1-4 oder mindestens 2 Symptome der Gruppen 5-8. Diese Symptome müssen fast ständig während eines Monats vorhanden sein (Sielk und Janssen 2004). 
Tabelle 1: Symptome zur Diagnosesicherung der Schizophrenie nach AWMFLeitlinien: Deutsche Gesellschaft für Psychiatrie, Psychotherapie und Nervenheilkunde (2005)

\section{Gruppe}

Symptome

Eindeutige Symptome:

1 Gedankenlautwerden, Gedankeneingebung, Gedankenentzug, Gedankenausbreitung

2 Kontrollwahn, Beeinflussungswahn, Gefühl des Gemachten bzgl. Körperbewegung, Gedanken, Tätigkeiten oder Empfindungen, Wahnwahrnehmungen

3 kommentierte oder dialogische Stimmen

$4 \quad$ völlig unrealistischer Wahn

Nichteindeutige Symptome:

5 anhaltende Halluzination jeder Sinnesmodalität

$6 \quad$ Zerfahrenheit durch Gedankenabreißen, Einschiebungen in den Gedankenfluss oder Neologismen

$7 \quad$ katatone Symptome wie Erregung, Haltungsstereotypien, Negativismus oder Stupor

$8 \quad$ Negativsymtome wie: Apathie, Sprachverarmung, verflachte oder inadäquate Affekte

\subsection{Epidemiologie}

Das Risiko, im Laufe des Lebens mindestens einmal an einer schizophrenen Episode zu erkranken (Langzeitprävalenz), liegt weltweit zwischen 0,3 und 2,7 \% (Saha et al. 2005). McGrath et al. (2004) kamen in ihrem Reviewartikel, in den 161 Studien eingingen, zu dem Ergebnis, dass die Inzidenz weltweit (Anzahl neuer Krankheitsfälle pro Jahr) zwischen 0.7 und 43.0 pro 100.000 Einwohnern liegt. Gleichzeitig wiesen sie auf eine höhere Inzidenz bei Männern und der städtischen Bevölkerung hin. Die Prävalenz (Krankheitshäufigkeit zum Zeitpunkt der Untersuchung) wird im Mittel mit 4.6 (1.9-10.0) Fällen pro 1.000 Einwohner weltweit angeben (Saha et al. 2005). In einigen Arbeiten wurde auf die überproportionale Häufigkeit in niedrigen sozialen Schichten hingewiesen, ein Befund, der in späteren Nachuntersuchungen zumeist damit erklärt werden konnte, dass Schizophrene im Verlauf ihrer Erkrankung sozial absteigen (Möller et al. 2005). Einen weiteren Erklärungsversuch für die Tatsache, 
dass schizophrene Patienten häufig einen niedrigen Bildungsstand und sozialen Status aufweisen, lieferten Wobrock et al. (2004). Sie gehen davon aus, dass es durch die Krankheit selbst bereits früh zu einer Beeinträchtigung der sozialen Entwicklung kommen kann.

Die Erkrankung tritt bei Männern im Mittel um das 21. Lebensjahr und bei Frauen etwa fünf Jahre später auf (Möller et al. 2005). Der Verlauf der Erkrankung stellt sich recht unterschiedlich dar. Man kann die Krankheit nach ihren Verläufen differenzieren: kontinuierlich, episodisch mit zunehmendem Residuum, episodisch mit stabilem Residuum, episodisch remittierend, unvollständige Remission, vollständige Remission. Als Faustregel gilt: ein Drittel der Patienten lebt relativ ungestört, ein Drittel zeigt eine deutliche Symptomatik, bleibt aber sozial integriert und ein Drittel hat einen chronisch progredienten Verlauf. Ungefähr $20 \%$ der Erkrankten zeigen eine vollständige Remission (AWMF-Leitlinien: Deutsche Gesellschaft für Psychiatrie, Psychotherapie und Nervenheilkunde 2005).

\section{3. Ätiopathologie}

Bei der Schizophrenie handelt es sich um ein Krankheitsbild mit multifaktorieller Genese, d.h. es müssen neurobiologische, psychologische und soziale Teilfaktoren berücksichtigt werden (Gaebel und Falkai 2003).

Eine genetische Komponente (polygener Erbgang) ist durch Familien-, Adoptionsstudien- und Zwillingsstudien belegt. Das Krankheitsrisiko ist um 10-15 \% erhöht, wenn nur ein Familienmitglied an Schizophrenie leidet (Wobrock et al. 2004). Sind beide Elternteile von der Krankheit betroffen, liegt das Risiko schon bei etwa 50 \% (Schultz und Andreasen 1999). Für die familiäre Häufung der Schizophrenie sind mit hoher Wahrscheinlichkeit mehrere untereinander interagierende Genkonstellationen verantwortlich, von denen jede nur einen kleinen Teil zum Erkrankungsrisiko beiträgt. Bisher konnten die Chromosomenabschnitte 5q, 6p, 8p, 10p, 13q, 18p und 22q als Kandidantenregionen für schizophrene Psychosen nachgewiesen werden. Die Identifikation erster risikomodulierender Gene (z.B. für Neuregulin und Dysbindin) ist gelungen (Falkai und Maier 2006).

Nicht-genetisch vermittelte Faktoren wie Schwangerschafts- und Geburtskomplikationen erhöhen das Risiko, an Schizophrenie zu erkranken, um ca. 1-2 \%. Weitere bestätigte Umweltfaktoren sind Drogenkonsum, Ernährungsdefizite, prä- und perinatale Virusinfekte (Schultz und Andreasen 1999). Solche früh erworbenen Dispositio- 
nen sind meist nicht allein für den Ausbruch der Krankheit verantwortlich, sondern andere Faktoren müssen hinzutreten. Sog. „High-risk-Studien“ an Kindern mit einem schizophrenen Elternteil zeigen, dass bereits in frühester Kindheit das soziokommunikative und motorische Verhalten späterer Schizophrener auffällig gestört ist. Dies trägt zur Entwicklung einer gestörten prämorbiden Persönlichkeit mit häufig abnormem Interaktionsstil bei. Ein höheres Rückfallrisiko wird Schizophrenen aus Familien mit „high expresses emotions“, d. h. emotionalem Überengagement mit erhöhtem Kritikverhalten zugeschrieben (Gaebel und Falkai 2003). Das Risiko, an Schizophrenie zu erkranken, kann durch weitere Faktoren, die in der Kindheit auftreten, wie Verlust eines Elternteiles, körperliche Misshandlung und sexueller Missbrauch erhöht werden (Wobrock et al. 2004).

Aus biochemischer Sicht wird als wichtigstes Korrelat akuter schizophrener Psychosen die Überaktivität dopaminer Strukturen diskutiert (Möller et al. 2005). Die Dopamin-Hypothese beruht auf der Beobachtung, dass Neuroleptika zentrale DopaminD2-Rezeptoren blockieren. Zudem können dopaminerge Substanzen wie z.B. Amphetamin (bewirkt eine Dopaminfreisetzung) eine akute Psychose induzieren, die der schizophrenen Symptomatik im Hinblick auf einzelne Symptome ähnelt (Heinz 2000). Dem serotonergen System wird ebenfalls eine entscheidende Rolle bei der Manifestation zugesprochen. Dies stützt sich vor allem auf die Beobachtung, dass unter der Einnahme von strukturähnlichen psychoaktiven Substanzen wie Lysergsäurediethylamid (LSD) regelhaft psychotische Symptome hervorgerufen werden. Die GlutamatHypothese der Schizophrenie geht davon aus, dass schizophrene Störungen durch eine Unterfunktion des glutamatergen kortikostriatalen und kortikomesolimbischen Systems hervorgerufen werden. Auch Veränderungen im GABA-Stoffwechsel und im Acetylcholin-System werden diskutiert (Wobrock et al. 2004).

Als weitere Ursachen der Schizophrenie werden hirnstrukturelle Veränderungen diskutiert. Hier liegt vermutlich als morphologische Grundlage eine Hirnentwicklungsstörung vor, die funktionell mit einer reduzierten Informationsverarbeitungskapazität einhergeht. Zur Erfassung der volumetrischen Veränderungen bei schizophrenen Patienten wurde eine Vielzahl von Studien durchgeführt. Bei einem Teil der Studien zeigte sich eine Abnahme des Gesamtvolumens des Gehirns, wobei die graue Substanz im Temporallappen am stärksten betroffen war. In den meisten Studien konnte eine Ventrikelerweiterung mit Schwerpunkt im Bereich der Temporalhörner und der Seitenventrikel gefunden werden (Wobrock et al. 2004). 


\subsection{Therapie}

Die Therapie der Schizophrenie stützt sich auf drei Säulen: Psychopharmakologie, Psychotherapie und Soziotherapie.

In der Akutbehandlung steht die Remission oder Suppression der Positivsymptomatik und die Verhütung der Fremd- oder Selbstgefährdung im Vordergrund (Gaebel und Falkai 2003). Bei allen Subtypen ist in dieser Phase, meist unter stationären Bedingungen, der Einsatz von konventionellen und atypischen Neuroleptika indiziert, die nach 4- bis 6-wöchiger Behandlung in 60-70 \% der Fälle zu einer deutlichen Symptomreduktion führen (Sielk und Janssen 2004). Überwiegt die Negativsymptomatik, sind atypische Neuroleptika zu bevorzugen. Die rechtzeitige Behandlung einer Erstmanifestation hat einen entscheidenden Einfluss auf die Langzeitprognose und kann einer Chronifizierung entgegenwirken. Auch in der Langzeitbehandlung, deren Therapieziele vor allem Rezidivprophylaxe oder anhaltende Symptomsuppression sind, stellen Neuroleptika die Basistherapie dar. Nach den AWMF-Leitlinien der Deutschen Gesellschaft für Psychiatrie, Psychotherapie und Nervenheilkunde (2005) sind die Medikamente mindestens 12 Monate lang nach einer Erstmanifestation zu geben. Wenn zwei oder mehr Rezidive aufgetreten sind, sollten Neuroleptika mindestens zwei oder fünf Jahre lang eingenommen werden, ggf. auch ein Leben lang.

Die zweite Säule der Behandlungsstrategien umfasst Psychotherapieverfahren mit Einzel- und Gruppenbehandlungen. Der therapeutische Schwerpunkt liegt auf Information (z.B. über Krankheitsmodelle), Edukation (z.B. über Rückfallerkennung und Behandlungserfordernisse), Training (z.B. kognitiver und sozialer Fertigkeiten) und Beratung (Sielk und Janssen 2004). Aufdeckende Therapieverfahren, wie analytische Psychotherapie und Gestalttherapie, können zur Symptomprovokation führen und sollten nur in speziellen Einzelfällen angewandt werden. In die Therapie sollte auch das familiäre Umfeld mit einbezogen werden (Gaebel und Falkai 2003).

Soziotherapeutische Maßnahmen stellen die dritte Säule in der Behandlung von schizophrenen Patienten dar. Wegen der Neigung von Schizophreniepatienten zu Hospitalisierungsschäden bei reizarmer Umgebung und wegen der Gefahr der Entwicklung chronischer Negativsymptomatik sind soziotherapeutische Maßnahmen besonders wichtig. Damit sollen vorhandene soziale Fähigkeiten des Erkrankten gefördert und die Entstehung bzw. Verstärkung sozialer Defizite verhindert werden. Neben der Arbeits- und Beschäftigungstherapie umfasst die Soziotherapie vor allem auch 
die Arbeit an Milieufaktoren und die Strukturierung des Tagesablaufes (Möller et al. 2005).

\subsection{Die ambulante Behandlung schizophrener Patienten}

Im Zuge epidemiologischer Forschungen wurde zunehmend deutlich, dass dem Allgemeinarzt eine wesentliche Rolle bei der Behandlung psychisch Kranker zukommt. Etwa 90 \% der Bevölkerung befinden sich in hausärztlicher Behandlung. Bei entsprechenden gesicherten Annahmen zur Prävalenz psychischer Störungen und einer bekanntlich höheren Inanspruchnahme psychisch Kranker kann man davon ausgehen, dass im Durchschnitt 15-25 \% der allgemeinärztlichen Patienten an akuten und behandlungsbedürftigen, zumindest aber an belastenden psychischen Störungen leiden (Linden et al. 1996).

Die quantitative Bedeutung und Belastung des Hausarztes in diesem Bereich ergibt sich auch aus Studien über die hausärztliche Betreuung schizophrener Patienten. So belegte eine Studie aus Irland (Gavin et al. 2005), dass 22 \% der schizophrenen Patienten ausschließlich durch den Allgemeinarzt betreut werden, in Australien sind es bis zu 31 \% (Lewin und Carr 1998). Eine Studie aus Mannheim und Oberbayern zeigte, dass $31 \%$ der schizophrenen Patienten allein durch den Hausarzt behandelt werden (Haasen et al. 1993). In einer neueren deutschen Veröffentlichung im Rahmen des Kompetenznetzes Schizophrenie wurden 10 \% der Schizophreniepatienten allein durch den Hausarzt versorgt (Abholz und Sielk 2003). Gleichzeitig kristallisierte sich in dieser Studie heraus, dass sich in erster Linie die Nervenärzte für die Behandlung schizophrener Patienten zuständig fühlen. So waren sich die hier befragten Nervenärzte einig, dass die Langzeitbetreuung von schizophrenen Patienten durch Fachärzte übernommen und nur in speziellen Ausnahmefällen dem Hausarzt überlassen werden sollte. Fast spiegelbildlich bezweifelten die meisten der befragten Hausärzte dieser Studie ihre eigene Kompetenz auf diesem Gebiet, waren primär jedoch auch nicht bereit, zeitintensive Fortbildungen zu diesem Thema zu absolvieren. Ähnliches wurde auch von Simon et al. (2005) und Lester et al. (2005) beschrieben.

In einer weiteren Studie im Rahmen des Kompetenznetzes Schizophrenie wurden Interviews mit Betroffenen und Angehörigen speziell zur Frage, aus welchen Gründen Patienten zum Hausarzt gehen, geführt. Hier suchten die Patienten den Hausarzt primär wegen somatischer Probleme auf und gingen mit psychischen Beschwer- 
den und bei Fragen bezüglich der Medikation zum Facharzt. Der Hausarzt wurde in der Rolle des Lotsen bzw. des Koordinators gesehen; gleichzeitig wurde die oft langjährige und vertraute Beziehung zum Hausarzt von den Patienten geschätzt (Himmel et al. 2003).

Eine ähnliche Studie führten Haasen und Stark (1997) mit hospitalisierten Patienten zur Inanspruchnahme des Hausarztes durch: Hauptgründe, warum der Hausarzt aufgesucht wurde, waren in 59 \% der Fälle somatische Beschwerden, 22 \% suchten ihn wegen psychischer Verschlechterung auf und $15 \%$, weil sie ein Rezept für Psychopharmaka brauchten. Dagegen wurde der Nervenarzt in $46 \%$ der Fälle regelmäßig besucht, während $23 \%$ inn besuchten, weil es ihnen psychisch schlechter ging und $19 \%$, weil sie ein Rezept für Psychopharmaka brauchten. Gleichzeitig ließen Haasen und Stark (1997) die beiden Arztgruppen durch die Patienten bewerten. Hier zeigte sich, dass die Schizophreniepatienten, die sowohl vom Hausarzt als auch vom Nervenarzt parallel betreut wurden, die Effizienz der Behandlung beim Nervenarzt höher bewerteten. Auch in der englischsprachigen Literatur finden sich Hinweise darauf, dass der Hausarzt primär wegen körperlicher Beschwerden aufgesucht wird. Bei Bindman et al. (1997) taten dies $75 \%$ der Patienten, und bei $20 \%$ war der Hausarzt mit in die psychiatrische Erkrankung einbezogen. In der Studie von Nazareth et al. (1995) suchten 40 \% den Hausarzt wegen körperlicher Beschwerden auf, $8 \%$ kamen hier wegen psychischer Probleme und $6 \%$ wegen ihrer Depotmedikamente.

Dem Hausarzt kommt bei der Schizophreniebehandlung vor allem in der Frühphase und der Remissionsphase eine zentrale Rolle zu, wogegen die akute Episode fachärztlich und in der Regel stationär therapiert wird. Der Beginn einer akuten Episode erfordert jedoch das Eingreifen des Hausarztes in Form der Modifikation der Therapie und gegebenenfalls die fachärztliche Überweisung oder stationäre Einweisung (Eich 1990). Gmür (1983 S. 1356) ist der Auffassung, dass sich bei leichten Krisen und bei Früh- und Residualsymptomen dem Hausarzt wichtige therapeutische Möglichkeiten bieten. Das sind unter anderem:

- _Einleiten einer antipsychotischen-neuroleptischen Medikation“

- $\quad$ „Entlastung am Arbeitsplatz und in der Familie durch Vermittlung der Tagesstruktur für den Patienten“

- $\quad$ „Besprechung der fassbaren und psychosozialen Konflikte in der Familie und der übrigen Umwelt“. 
Auch in den Arbeiten von Köhn et al. (2004 b) und Fuchs und Steinert (2004) zum Hilfesuchverhalten schizophrener Ersterkrankter in Deutschland wurde deutlich, dass dem Hausarzt gerade in der Frühphase der Erkrankung eine wichtige Bedeutung zukommt. Sie zeigten, dass $25 \%$ der an Schizophrenie Ersterkrankten zuerst den Hausarzt aufsuchen. Gleiches fand sich in der Schweiz. Hier suchten ebenfalls $25 \%$ der Schizophrenieneuerkrankten zuerst Hilfe beim Hausarzt (Platz et al. 2006).

Auch andere Autoren sind der Meinung, dass die Früherkennung der Erkrankung eine wichtige hausärztliche Aufgabe und das rechtzeitige Erkennen wesentlich mitbestimmend ist für einen weiteren positiven Verlauf der Erkrankung (Ruhrmann et al. 2003, Aston et al. 2002, Bottlender und Möller 2004, Jäger et al. 2006, Vauth et al. 2004). Um dem Hausarzt diese Aufgabe zu erleichtern, wurden Risiko-Checklisten für beginnende Psychosen speziell für den hausärztlichen Bereich entwickelt (Aston et al. 2002, Bottlender und Möller 2004, Maurer et al. 2006).

Vor allem Frauen, Ältere, Bewohner ländlicher Gegenden und Verwitwete mit psychischen Erkrankungen werden alleinig vom Hausarzt betreut. Die Komorbidität mit anderen Erkrankungen und die damit verbundene höhere Frequenz an Gesprächen führten nach Meller et al. (1990) zu einer geringeren Notwendigkeit einer Überweisung. Besonders im ländlichen Gebiet kann die Distanz von der Wohnung zur ambulanten Einrichtung bei Vorliegen körperlicher Beschwerden ein wesentliches Hindernis darstellen, so dass häufig der Allgemeinarzt die Behandlung übernehmen muss (Meller et al. 1990).

Der Hausarzt übernimmt neben der Betreuung der psychischen Erkrankung die übliche Krankenversorgung (Haasen und Fritsche 1992, Vauth et al. 2004), die unabhängig oder bei einer Begleiterkrankung der Schizophrenie (z.B. komorbide Sucht, Arzneimittelnebenwirkungen) nötig werden kann, sowie die regelmäßigen ärztlichen Untersuchungen (Labor, EKG, EEG) unter der Therapie mit Antipsychotika (Jäger et al. 2006).

\subsection{Kriterien der Arztwahl und Arztbewertung psychisch Kranker}

Gemäß einigen der bereits zitierten Studien befindet sich teilweise bis zu einem Drittel der an Schizophrenie Erkrankten in alleiniger Behandlung des Hausarztes. Welche Vorteile könnte eine Behandlung durch den Hausarzt für den Patienten bieten? Im Vergleich zum Psychiater kennt der Hausarzt den Patienten seit Jahren, meist auch seine Familie und ist im besten Sinne „Familienarzt“ (Abholz und Kochen 2006, 
Himmel et al. 2006, Sielk und Janssen 2004). Der Arzt lebt meist im gleichen Ort wie der Patient und kennt die familiären und lokalen Ressourcen besser. Häufig besitzt der Hausarzt einen Vertrauenskredit sowohl vom Patienten selbst als auch von seiner Familie (Eich 1990, Aston et al. 2002). Dadurch hat er die Chance, Langzeitentwicklungen des Patienten wahrzunehmen und zu beeinflussen (Vauth et al. 2004, Aston et al. 2002). Er kann am besten den aktuellen gesundheitlichen Zustand einschätzen sowie eventuelle Frühsymptome erkennen, welche auf eine drohende psychotische Exazerbation hinweisen, und entsprechend handeln (Vauth et al. 2004).

Ein weiterer Grund, weshalb Patienten zunächst ihren Hausarzt aufsuchen, könnte sein, dass eine psychiatrische Diagnose sich diskriminierend auf die Wahrnehmung der Patienten auswirken könnte und der Allgemeinarzt bei leichten psychiatrischen Störungen diese primär nicht definiert, auch wenn er die Störung als solche erkannt hat. Nach Angaben von Sielk und Abholz (2005) diagnostizieren Hausärzte einige psychische Störungen nicht explizit, lassen diesen aber implizit in der Konsultation einen breiten Raum und „behandeln“ sie mit, ohne dass dies so benannt wird. Linden et al. (1996) wiesen daraufhin, dass unter Praxisbedingungen Diagnosen eine operationale Funktion haben. Sich um eine Diagnosestellung zu bemühen, macht nur dann Sinn, wenn sich daraus therapeutische Konsequenzen ergeben. Dies mag aus Sicht der Allgemeinärzte bei grenzwertigen psychischen Störungen nicht unbedingt der Fall sein (Abholz 2006). Wird die psychiatrische Diagnose gestellt und eine entsprechende Therapie eingeleitet, stößt das in manchen Fällen auf Ablehnung durch die Patienten. Nach Meinung von Sielk und Abholz (2005) können Ärzte in eine Konfliktsituation mit Patienten geraten, wenn die Therapie dennoch durchgesetzt werden soll. Um das primär zu vermeiden, stellen Hausärzte bei leichteren psychischen Störungen die mögliche „Diagnose“ vielleicht deshalb bewusst nicht.

Die Formulierung psychiatrischer Diagnosen ist zum einen abhängig von der Erkennungsrate der Symptomträger und zum anderen von der Negativassoziation, die mit einer bestimmten Diagnose, z.B. Schizophrenie, verbunden ist (Stark und Stolle 1994). Es ist anzunehmen, dass die Hemmschwelle des Patienten, bei psychischen Störungen primär einen Psychiater aufzusuchen, höher liegt als die, seine Probleme zunächst dem Hausarzt vorzutragen (Aston et al. 2002, Vauth et al. 2004).

Wie bereits festgestellt, werden vor allem Frauen, Ältere und Bewohner ländlicher Gegenden vorwiegend vom Allgemeinarzt alleinig betreut (Meller et al. 1990). Gründe für die niedrige Inanspruchnahme eines Spezialisten von über 75-Jährigen könnten die Haltung und Einstellung des Allgemeinarztes hinsichtlich einer Überweisung 
an den psychischen Spezialisten sein. Dass Stigmatisierung bei „Älteren“ stärker ins Gewicht fällt und umgekehrt psychische Störungen im Alter - da vergleichsweise häufig - eher für „normal“ erachtet werden (Meller et al. 1990), können weitere Gründe für die Präferenz von Allgemeinärzten sein.

In Neuseeland wurde der Frage nachgegangen, in welcher Rolle sich die Hausärzte selbst bei der Behandlung psychisch Kranker sehen. Fast alle Ärzte sahen ihre Hauptaufgabe darin, körperliches Leiden der Patienten zu behandeln und die Patienten wegen ihrer psychischen Probleme zum Spezialisten zu überweisen; $94 \%$ fanden es jedoch wichtig, als „Co-Manager" bei psychischen Problemen zu fungieren. Schwierigkeiten bei der Behandlung psychisch Kranker sahen $79 \%$ darin, dass ihnen die Zeit für die Behandlung fehlt und dass häufig eine ineffektive Zusammenarbeit zwischen den Allgemeinärzten und Fachspezialisten herrscht. Fast $70 \%$ der Ärzte fühlten sich nicht ausreichend über Therapieänderungen informiert (Falloon et al. 1996). Zu ähnlichen Ergebnissen kamen auch andere Autoren: Hausärzte fühlen sich meist für die körperlichen Beschwerden zuständig (Abholz und Sielk 2003, Simon et al. 2005, Lester et al. 2005, Nazareth et al. 1995) und geben zum Teil Wissensdefizite in der Schizophreniebehandlung zu (Abholz uns Sielk 2003, Simon et al. 2005).

In den letzten Jahrzehnten hat sich die Rolle des Patienten vom passiven Zuhörer zum aktiven Konsumenten gewandelt. Der „moderne Patient“ will mit in die Entscheidungen der Behandlung einbezogen werden und erwartet eine Aufklärung über seinen Gesundheitszustand (Schattner et al. 2006, Carlsen und Aakvik 2006, Vedstedt et al. 2002, Hansson et al. 1993). Campbell et al. (2007) kamen in ihrer Erhebung zu dem Ergebnis, dass Zufriedenheit mit dem Arzt positiv durch ausreichenden Informationserhalt und durch Mitspracherecht beeinflusst wird. Steht der Arzt offen einem Mitspracherecht seitens der Patienten gegenüber, wächst die Patientenzufriedenheit (Carlsen und Aakvik 2006). Wichtig für eine vertrauensvolle Zusammenarbeit zwischen Arzt und Patient ist es, dass der Arzt die Absicht erkennt, mit welcher der Patient den Arzt aufsucht (Salomon und Quine 1989).

Kravitz et al. (1994) befragten 304 Patienten von Klinikärzten und Hausärzten über ihre Erwartungen und Zufriedenheit beim Arztbesuch: Patienten waren am meisten unzufrieden, wenn eine erwartete medizinische Handlung unterblieb (Untersuchung eines bestimmten Organsystems, Rezept, Anordnung von Labortests u.a.). Ferner missfiel es ihnen, wenn nicht nach der familiären Vorgeschichte gefragt oder die Prognose der Erkrankung eingeschätzt wurde und wenn die eigenen Ideen des Pati- 
enten nicht mit in die Therapieplanung einbezogen wurden. Ein weiteres wesentliches Ergebnis dieser Studie war, dass Patienten mit unterschiedlichem sozialen Hintergrund auch unterschiedliche Erwartungen an den Arzt haben.

Zum Thema „Patientenbedürfnisse im Rahmen der medizinischen Versorgung“ liegen mehrere Studien vor. Van Campen et al. (1998) entwickelten zum Beispiel ein Instrument, um die Bedürfnisse und Erwartungen des individuellen Patienten zu erfahren und somit die Qualität des Gesundheitswesens aus der Perspektive des Konsumenten zu messen. In einer Hälfte des Fragebogens konnten Patienten allgemeine Aspekte der Arzt-Patienten-Beziehung, wie z.B. „der Arzt versteht mein Problem“ oder „er arbeitet effektiv“, beurteilen. Die andere Hälfte war spezifisch auf die Bedürfnisse von Rheumapatienten, wie z.B. „die Praxis ist behindertengerecht eingerichtet“, ausgelegt. So war es auch möglich, den Hausarzt und den Spezialisten bezüglich der Patientenbewertung direkt zu vergleichen. Ähnliche Fragebögen gibt es inzwischen für HIV-Patienten (Hekkink et al. 2003), für Asthma- Patienten (van Campen et al. 1997) und für ältere, multimorbide Patienten (Sixma et al. 2000).

Im Rahmen der Schizophrenieforschung finden sich einige Studien zum Thema, welche Bedürfnisse Schizophreniepatienten haben und von wem sie erfüllt werden sollten (Wiersma et al. 1998, Hansson et al. 1995, Hoffmann und Priebe 1996, Priebe et al. 1995, Kallert und Leisse 2000). In ihrer Arbeit deckten Bengtsson-Tops und Hansson (1999) auf, dass eine Unterversorgung bei der Information über den Gesundheitszustand, der Behandlung und der persönlichen Beziehung besteht - und das unabhängig vom Arzttyp.

Lester et al. (2003) beschäftigten sich mit der Frage, was Schizophreniepatienten bei der hausärztlichen Behandlung besonders wichtig ist. Hierzu führten sie halbstrukturierte Interviews mit 45 Patienten durch. Es zeigte sich, dass u.a. eine emphatische Gesprächssituation und die Persönlichkeit des Arztes wichtige Aspekte für eine hohe Zufriedenheit des Patienten sind. Am wichtigsten für diese Patientengruppe war jedoch, dass der Hausarzt innen Mut gibt und die Erkrankung nicht als unheilbar bezeichnet.

Cooper et al. (2000) befragten 76 depressive Patienten an einer Universitätsklinik zu ihrer Arztsicht. Auf Basis mehrerer Vorstudien wurde ein Fragebogen entwickelt, dessen Fragen (,Items“) 7 Hauptkriterien zugeordnet waren, u.a. persönliche Fähigkeiten der Arztes, Fähigkeiten zur Erkennung der Depression, Effektivität der Behandlung und Behandlungsprobleme. Am wichtigsten im Sinne einer guten Behand- 
lungsqualität war den Patienten, dass ihr Arzt über gute persönliche Fähigkeiten verfügt, die Symptome rechtzeitig und zuverlässig erkennt, ihnen Informationen liefert und die Therapie erfolgreich „managt“. Den ersten Platz auf der Rangliste nahm das Vertrauen in den Arzt ein.

Concato und Feinstein (1997) führten in Neuengland eine Studie mit 202 Patienten durch, von denen jeweils 50 \% von Hausärzten und Klinikärzten betreut wurden. Mit halboffenen Fragen wurden Wünsche des Patienten an das Gesundheitssystem (Ärzte, nichtärztliches Personal, Apotheken, Kliniken) erfasst. Auf das ärztliche Personal bezogen, waren für die Patienten Pünktlichkeit, Effizienz, Kompetenz und Erfahrung von Wichtigkeit. Ferner spielte der Kommunikationsstil eine große Rolle.

Im Bereich der nervenärztlichen Versorgung wurden in den letzen Jahren ebenfalls einige Studien durchgeführt. Hier wünschten sich die Patienten vor allem einen Nervenarzt, der sie respektiert, zuhört (Björkman et al. 1995) und informiert (Spießl et al. 2002, Björkman et al. 1995, Hansson et al. 1993).

Zahlreiche Autoren haben untersucht, welche Aspekte („Dimensionen“) für die Zufriedenheit von Patienten wichtig sind und wie man diese verlässlich misst. Patientenzufriedenheit kann durch eine gelungene Kommunikation gefördert werden. Little et al. (2001) führten fünf Komponenten an, die zu einer gelungenen Kommunikation beitragen:

- Erklärung der Erfahrung und Erwartung, die der Arzt mit der Krankheit hat

- Verstehen der ganzen Person

- Finden einer gemeinsamen Partnerschaftsebene

- Gesundheitsförderung

- Förderung der Arzt-Patienten-Beziehung.

In Norwegen wurden rund 2000 Patienten unter anderem zur Kommunikation mit ihrem Arzt befragt. Im Ergebnis dieser Arbeit zeigte sich, dass gerade in diesem Punkt noch großer Handlungsbedarf zur Verbesserung besteht: Fast die Hälfte der Befragten gab an, kein optimales Gespräch mit ihrem Arzt geführt zu haben, $70 \%$ sahen zumindest einige Kommunikationsbarrieren und $24 \%$ verließen die Praxis mit negativem Gefühl. Eine effektive Kommunikation war davon abhängig, inwieweit der Arzt die Patientenperspektive verstand. Immerhin $60 \%$ der befragten Studienteilnehmer waren trotz allem sehr zufrieden mit dem Arzt, wobei die kommunikativen Fähigkei- 
ten im Vergleich zu den technischen Fähigkeiten des Arztes höher bewertet wurden (Steine et al. 2001).

In den letzten Jahrzehnten haben verschiedene Arbeitsgruppen Fragebögen entwickelt, welche die Zufriedenheit der Patienten mit dem Arzt messen sollten (z.B. Gericke et al. 2004, Bitzer et al. 1999, Wolf et al. 1978, Concato und Feinstein 1997). Im hausärztlichen Bereich liegen dazu u.a. Arbeiten von Klingenberg et al. (1999), Grogan et al. (2000), Valori et al. (1996) und Grol et al. (2000) vor. Auch für die nervenärztliche Behandlung sind Erhebungsinstrumente zu dieser Thematik entstanden (z.B. Cooper et al. 2000, Barker und Orrell 1999, Gigantesco et al. 2003).

Im deutschsprachigen Raum stehen zur Zufriedenheitsmessung nur wenige validierte Instrumente zur Verfügung (Bitzer et al. 1999, Gericke et al. 2004, Klingenberg et al. 1999). Bitzer und Dierks (1999) sind im Rahmen des Verbundprojekts „Qualitätsmangement in der Arztpraxis“ der Frage nachgegangen, wie man die Zufriedenheit der Patienten mit der ambulanten Versorgung erfassen kann. Nach inhaltsanalytischer Auswertung von Gruppendiskussionen extrahierten sie vier Dimensionen („Fachliche Kompetenz des Arztes“, „Arzt-Patienten-Interaktion“, „Information“ und „Praxisorganisation“), die sich spezifisch auf den Versorgungsprozess und die für die Patienten erkennbaren und erlebbaren Prozesse in der ärztlichen Praxis beziehen. Die Arbeitsgruppe Gericke et al. (2004) entwickelte ein Erhebungsinstrument, das sowohl in der hausärztlichen als auch in der fachärztlichen Praxis Daten zur Patientenzufriedenheit mit der ambulanten medizinischen Versorgung messen kann - das Qualiskope-A. Der Fragebogen besteht aus 27 Einzelitems, welche die gleichen Dimensionen wie bei Bitzer und Dierks (1999) - nämlich „Fachliche Kompetenz des Arztes“, „Arzt-Patienten-Interaktion“, „Information“ und „Praxisorganisation“ - abdecken (Gericke et al. 2004).

Studien mit diesen Fragebögen belegen, dass ein Großteil der Patienten mit der Betreuung durch den Arzt zufrieden ist, aber dennoch in einigen Punkten Handlungsbedarf zur Verbesserung besteht.

So konnten Williams und Calnan (1991) zeigen, dass 95 \% der Patienten mit ihrem Arzt zufrieden sind, wenn die Fragen zur Erwartung an den Arzt sehr allgemein gestellt werden. Sobald die Fragen detaillierter wurden, zeigte sich jedoch Handlungsbedarf. Beispielsweise glaubten $38 \%$ der Patienten, mit ihrem Hausarzt nicht über persönliche Probleme sprechen zu können, 28 \% bemängelten die geringen Informationen vom Arzt und $25 \%$ waren damit unzufrieden, dass der Arzt zu wenig Zeit für 
sie hat. Ähnliches zeigte sich in einer Studie aus Oslo, in der 1193 Patienten mit rheumatischen Erkrankungen zur Zufriedenheit befragt wurden. Hier berichteten $12 \%$ der Teilnehmer, keine Information über die Diagnose und Medikamente erhalten zu haben, und 35 \% meinten, nicht genug Hilfestellung für den Alltag erhalten zu haben (Kjeken et al. 2006).

In der Umfrage von Grol et al. (2000) in 10 europäischen Ländern fühlten sich 80 \% der Studienteilnehmer von ihrem Hausarzt gut bis sehr gut behandelt. In Deutschland wurden im Rahmen dieser Studie aus 36 verschiedenen Praxen 2880 Patienten zu ihrer Zufriedenheit mit dem Hausarzt befragt, von denen $77 \%$ antworteten. Fast alle Befragten sahen keinen Grund, ihren Hausarzt zu wechseln. Auch wenn dies auf den ersten Blick eine sehr positive Bewertung des Hausarztes ist, waren aber wiederum bei den Detailfragen 77 \% der Befragten nicht ganz zufrieden damit, wie ihr Arzt sich an frühere Gespräche und Behandlungen erinnert; 69 \% konnten sich eine bessere Unterstützung im Umgang mit ihren Gefühlen vorstellen. Über 60 \% der Befragungsteilnehmer konnten sich vorstellen, besser in Entscheidungen einbezogen zu werden - für den betreffenden Arzt ein wichtiger Hinweis auf ein mögliches Unzufriedenheitspotential. Ebenso ist es für den Hausarzt wichtig zu wissen, dass im Durchschnitt zwei Drittel seiner Patienten nicht sicher sind, wie wichtig die Befolgung ärztlicher Ratschläge ist. Einige Patienten bemängelten auch die langen Wartezeiten, Fehlen notwendiger technischer Geräte und „den ersten Griff zum Computer" (Klingenberg et al. 1999). Diese Kritik teilten auch Patienten anderer europäischer Nationen. Als negativ wurden die langen Wartezeiten und die Erreichbarkeit des Hausarztes empfunden. Positiv bewerteten die Patienten die vertrauliche Behandlung ihrer Daten und die Tatsache, dass sie mit ihrem Arzt über Probleme reden können und er sich entsprechend Zeit für sie nimmt (Grol et al. 2000).

Befragungen von Schizophreniepatienten (Ruggeri et al. 2003, Schildmann et al. 2003) und von Patienten mit unterschiedlichsten psychiatrischen Diagnosen (Hansson 1989, Gigantesco et al. 2003, Thapinta et al. 2004) zeigten ebenfalls, dass in einigen Bereichen noch Verbesserungsbedarf besteht, vor allem in der Vermittlung von Informationen.

\subsection{Zusammenfassung}

Die Sichtung der Literatur der vergangenen Jahre insbesondere zu den Kriterien der Arztwahl ergab, dass das Thema von einigen Autoren aufgegriffen wurde und mit 
verschiedenen Instrumenten (standardisierter Fragebogen, offene Fragen, halboffene Fragen) bearbeitet wurde. In diese Studien gingen Patientenstichproben mit unterschiedlichsten Diagnosen ein, es erfolgte selten eine Diagnosespezifizierung.

Die Erkrankung Schizophrenie unter dem Aspekt der Arzt-Patienten-Beziehung war gleichfalls Ziel von Forschungsarbeiten der letzten Jahre. Allerdings begrenzte sich die Fragestellung auf die Häufigkeit der Inanspruchnahme im Vergleich von Hausarzt und Psychiater, Verifizierung von Diagnosen und die Rolle des Arztes bei der Behandlung. Die Datenlage zu den subjektiven Kriterien der Patienten zur Inanspruchnahme des Arztes ist dagegen relativ spärlich. Wünsche und Bewertungen des Arztes aus Patientensicht fanden sich nur bei wenigen Autoren (Haasen und Stark 1997, Lester et al. 2003, Himmel et al. 2003, Schildmann et al. 2003, Ruggeri et al. 2003). Bei der Studie von Himmel et al. (2003) im Rahmen des Kompetenznetzes Schizophrenie wurden im freien Interview die Betreuungssituation im Einzelnen sowie die Wünsche einer kleinen Stichprobe von Patienten und ihre Erwartungen an den Arzt erfragt. Wünschenswert wäre es, mit einer größeren Stichprobe und mit einem standardisierten Erhebungsinstrument gezielt die Patientenwünsche und Erwartungen an Hausärzte bzw. Fachärzte genauer zu untersuchen und gleichzeitig zu erheben, wie diese Patienten ihre derzeitige ambulante Betreuungssituation bewerten. 


\section{Fragestellung und Ziel}

In der vorliegenden Arbeit sollten die Kriterien bestimmt werden, nach denen Patienten mit der Krankheit Schizophrenie einen Arzt zur Behandlung und Betreuung auswählen und bewerten. Im Mittelpunkt stand die Frage, welche Bedeutung Hausärzte und Psychiater/Neurologen für die Patienten haben, insbesondere in der Langzeitbetreuung. Im Einzelnen sollten folgende Aspekte näher betrachtet werden:

- $\quad$ Gehen Patienten vorwiegend wegen somatischer Beschwerden zum Hausarzt oder spielen auch psychiatrische Aspekte eine Rolle?

- $\quad$ Erwarten Patienten von ihrem Hausarzt überhaupt eine Behandlung bzw. Mitbehandlung ihrer psychiatrischen Symptome und halten sie ihn für fachlich kompetent?

- $\quad$ Gibt es Gründe, weshalb Patienten wegen ihrer psychischen Beschwerden lieber zum Hausarzt als zum Facharzt gehen?

- $\quad$ Sieht der Patient den Psychiater als den geeigneteren Arzt für die Behandlung seiner psychiatrischen Erkrankung und, wenn ja, aus welchen Gründen?

- $\quad$ Welche Kriterien sehen schizophrene Patienten bei der Bewertung von Hausarzt und Psychiater als relevant an?

- $\quad$ Wie bewerten schizophrene Patienten ihren derzeitigen Hausarzt bzw. Psychiater? 


\section{Material und Methoden}

In der vorliegenden Arbeit wurden schizophrene Patienten mit Hilfe eines selbst entwickelten Fragebogens zu ihren Erwartungen an ihren derzeitigen Hausarzt bzw. Nervenarzt interviewt. Außerdem sollten die Patienten die Betreuungsqualität durch beide Fachgruppen einschätzen. Ergänzt wurde das Interview durch einige offene Fragen. Die Patienten wurden über ambulante Ärzte, stationäre Einrichtungen und Behindertenwerkstätten kontaktiert.

\subsection{Untersuchungsorte}

Die Befragung der Patienten erfolgte in zwei unterschiedlichen Regionen Deutschlands: (1) in Bad Driburg, einer Stadt in Nordrhein-Westfalen, sowie (2) in Eisenach und dem angrenzenden Wartburgkreis in Thüringen.

Bad Driburg, zum Kreis Höxter gehörend, hat fast 20.000 Einwohner und verfügt neben 8 RehaKliniken über ein katholisches Krankenhaus - das St. Josef-Hospital (Bad Driburger Touristik GmbH 2007).

Die Geschichte des St. Josef-Hospitals lässt sich bis 1864 zurückverfolgen. Zu dieser Zeit verfügte das Krankenhaus lediglich über 12 Betten, doch bereits im Jahre 1879 wurde es unter dem Namen „Josephinum“ als Driburger Belegkrankenhaus mit 80 Betten geführt. Um den modernen Anforderungen der Zeit gerecht zu werden, wurde 1967 das jetzige St. Josef-Hospital bezogen. Zu den bereits integrierten Fachbereichen wurde 1968 eine Abteilung für Klinische Psychiatrie angegliedert. Damit war in Deutschland die erste psychiatrische Abteilung geschaffen, die eine Vollversorgung eines Kreises als Abteilung eines Allgemeinkrankenhauses übernahm. Derzeit verfügt die psychiatrische Abteilung über ungefähr 80 Betten. Dem stationären Bereich ist eine Ambulanz angeschlossen sowie eine Tagesklinik, die für ungefähr 30 Patienten ausgelegt ist.

Zum Behandlungsangebot gehören Einzel- und Gruppenpsychotherapie sowohl mit tiefenpsychologischer als auch mit kognitiv-verhaltenstherapeutischer Grundorientierung. Des Weiteren werden therapeutische Maßnahmen wie Entspannungstraining, Musiktherapie und Psychodrama angeboten. Für ältere Patienten und Abhängigkeitskranke gibt es spezielle Therapiegruppen. Zweimal wöchentlich besteht für Patienten mit Schizophrenie die Möglichkeit, im Tageszentrum zusammenzukommen, um an einer Schulung über ihr Krankheitsbild teilzunehmen (St. Josef-Hospital Bad Driburg 2007).

In die Studie wurden Patienten aus dem stationären Bereich, der Ambulanz und der Tagesklinik des St. Josef-Hospitals einbezogen. Als weitere Einrichtung für die Kontaktierung der Patienten wurde die gemeinnützige Integ $\mathrm{GmbH}$ in Bad Driburg ausgewählt. 
Die Integ wurde 1974 auf Initiative des leitenden Arztes der psychiatrischen Abteilung des St. JosefHospitals in Bad Driburg und des katholischen Gemeindepfarrers mit der Zielsetzung gegründet, „ (...) Menschen mit Behinderungen, die derzeit auf dem aktuellen Arbeitsmarkt nicht vermittelbar sind, eine Integration in das Berufsleben durch angepasste Arbeitsmethoden und Arbeitsplätze zu ermöglichen und somit die Möglichkeit einer gesellschaftlichen Integration der behinderten Menschen zu erreichen" (Präsentation der Integ gGmbH 2003, S. 2). Inzwischen finden in der Behindertenwerkstatt 580 Mitarbeiter Arbeit, wovon 363 Beschäftigte an einer psychischen, geistigen oder körperlichen Krankheit leiden (Präsentation der Integ gGmbH 2007). Integ unterscheidet sich von vielen anderen Behindertenwerkstätten durch eine leistungsgerechte Entlohnung. So sind je nach Leistungsniveau Löhne zwischen 0,40 Euro/Stunde und 11,50 Euro/Stunde erreichbar. Möglich ist dies allerdings nur, weil neben den behinderten Beschäftigten ungefähr ein Viertel bis ein Drittel der Gesamtarbeitnehmer gewerbliche Mitarbeiter sind (Präsentation der Integ gGmbH 2003).

Als zweiter Befragungsort wurde die Stadt Eisenach und der angrenzende Wartburgkreis ausgewählt.

Die Grundversorgung der 44.000 Einwohner der kreisfreien Stadt Eisenach erfolgt durch 23 niedergelassene Hausärzte und im stationären Bereich durch das St. Georg Klinikum Eisenach (Stadtportrait Eisenach 2007). Dieses entstand im April 2002 aus der Fusion des ehemaligen Christlichen Krankenhauses und des Wartburgklinikums Eisenach. Derzeit verfügt das Krankenhaus über 385 Planbetten, die sich auf neun verschiedene Fachrichtungen aufteilen. Dem St. Georg Klinikum ist eine Tagesklinik für Psychiatrie und Psychotherapie angeschlossen, in der 30 Patienten mit unterschiedlichen psychiatrischen Diagnosen betreut werden (St. Georg Klinikum Eisenach 2007). Außerhalb der Tagesklinik erfolgt die ambulante Versorgung von psychisch kranken Patienten im Raum Eisenach durch fünf niedergelassene Nervenärzte. Eine stationäre Behandlung für psychisch kranke Patienten ist in Eisenach nicht möglich. Patienten dieses Einzugsbereiches werden zum einen im ökumenischen Krankenhaus Mühlhausen (30 km nördlich von Eisenach) behandelt, zum anderem im Klinikum Bad Salzungen (25 $\mathrm{km}$ südlich von Eisenach).

Das 2002 neu erbaute Klinikum Bad Salzungen verfügt derzeit über 416 Betten, wovon 96 Betten auf die 1997 gegründete Psychiatrie- und Psychotherapieabteilung entfallen. Der psychiatrischen Abteilung ist eine Tagesklinik mit 15 Plätzen angeschlossen (Krankenhaus Bad Salzungen 2007).

In die vorliegende Studie wurden Patienten aus dem ambulanten Bereich der Hausund Nervenärzte in Eisenach sowie der Eisenacher Tagesklinik einbezogen. Im Raum Bad Salzungen wurden ambulante Patienten aus der Klinikambulanz sowie Patienten aus dem stationären psychiatrischen Bereich befragt. Als weiterer Zugangsweg für die Kontaktaufnahme mit den Patienten wurden 3 Behindertenwerkstätten in Eisenach und Umgebung genutzt:

(1) Die Hörselbergwerkstatt wurde 1995 mit 15 psychisch kranken Beschäftigten durch den Diakonieverbund in Eisenach gegründet. Derzeit finden hier 151 psychisch Kranke in zwei Teilwerkstätten in den Bereichen Schlosserei, mechanische Bearbeitung, Hausmeisterdienstleistungen, Montage, Ver- 
packung, Hauswirtschaft, Catering und der Cafeteria Arbeit (Präsentation der Hörselbergwerkstatt 2007).

(2) In der zweiten Behindertenwerkstatt, dem Bodelschwingh-Hof-Mechterstädt, dessen Träger ebenfalls der Diakonieverbund ist, werden mehr als 400 Beschäftigte betreut. Die Geschichte dieser Behindertenwerkstatt lässt sich bis 1949 zurückführen. Damals erhielt der Gärtnermeister Gustav Vogel von der evangelisch-lutherischen Kirche den Auftrag, brachliegendes Land in eine gemeinnützige Gärtnerei umzuwandeln (Bodelschwingh-Hof-Mechterstädt 2007).

(3) Die Diakonische Behindertenhilfe Bad Salzungen-Schmalkalden e. V. bietet derzeit mehr als 500 behinderten Menschen in vier Teilwerkstätten Arbeitsmöglichkeiten. In der Diakonie-Werkstatt Fambach, im Oktober 2001 gegründet, finden 60 ausschließlich psychisch kranke, seelisch behinderte Menschen Beschäftigung. Dieser Werkstatt ist eine Außenstelle in Bad Salzungen mit nochmals 30 psychisch kranken Beschäftigten angeschlossen (Diakonische Behindertenhilfe Bad Salzungen Schmalkalden e.V. 2007).

Diese Vielzahl von Zugangswegen sollte eine möglichst große Heterogenität der Befragten sicherstellen bzw. eine sehr einseitige Selektion mit Verzerrungseffekten einschränken.

\subsection{Erhebungsinstrument und Pilotstudie}

Im Vorfeld der Befragung wurde eine Pilotstudie in der Eisenacher Tagesklinik und in der Ambulanz in Bad Salzungen durchgeführt. Geprüft wurden hierbei zwei Varianten von Fragebögen unter dem Aspekt, welche der Varianten eine größere Akzeptanz bei den Patienten fand und für die Patienten besser verständlich war. Bei der Entscheidung, welcher Dokumentationsbogen endgültig verwendet wurde, musste berücksichtigt werden, dass die Patienten zum Zeitpunkt der Befragung unterschiedlich „krank" waren und ihr Auffassungsvermögen durch den Schweregrad der Erkrankung und eventuell unerwünschte Medikamentenwirkungen eingeschränkt war.

Wie bereits erwähnt, handelt es sich bei der Studie um eine standardisierte Befragung. Für diese Form der Befragung gelten bestimmte Regeln. Es sind „geschlossene“ Fragen zu verwenden, so dass der Befragte sich nur zwischen vorgegebenen Antwortmöglichkeiten zu entscheiden braucht. Weiterhin sind die Fragen in einer feststehenden Reihenfolge angeordnet, die bei der Durchführung nicht verändert werden darf (Scholl 2003). Da die Befragung persönlich in einem Einzelinterview erfolgte, spricht man auch von einem face-to-face-Interview (Bortz und Döring 2002). 
Für die Befragung wurden drei Fragebögen genutzt:

(1) Zu Beginn und am Ende der Befragung wurden jeweils zwei offene Fragen gestellt (siehe Kapitel 9.5 im Anhang; Einstiegs- und Abschlussfragen), die darüber Auskunft geben sollten, in welchen Situationen die Patienten zu welchem Arzt (Hausarzt/Nervenarzt) gehen, ob es Gründe für dieses Verhalten gibt und welche Eigenschaften Hausarzt bzw. Nervenarzt aus Sicht der Befragten haben sollten. Die Antworten wurden in Kategorien zusammengefasst.

(2) Der zweite Teil beinhaltete 44 Fragen mit je zwei Aspekten:

(a) Mit Hilfe des ersten Teilfragebogens (siehe Kapitel 9.6 im Anhang; Fragebogen Teil 1) sollte herausgefunden werden, welche Aspekte der ArztPatienten-Beziehung für die Befragten wichtig waren, unterschieden nach Hausarzt und Psychiater. Dazu wurden im Fragebogen in unterschiedlicher Reihenfolge Fragen zu Rahmenbedingungen der Praxis (u.a. Nähe des Wohnortes, Praxisatmosphäre, schnelle Erreichbarkeit, Hausbesuche, Wartezeiten), Fragen zur Persönlichkeit des Arztes und zu dessen fachlicher Kompetenz (u.a. sich Zeit nehmen, Ergebnisse der Untersuchung oder Behandlung erklären, Qualität der körperlichen Untersuchung, Fachwissen zur seelischer Erkrankung) sowie zur emotionalen Beziehung zwischen Arzt und Patient (u.a. Vertrauen, Ansprechpartner für private Probleme, Akzeptanz) gestellt. Für jedes Item wurden vier identische Antwortmöglichkeiten vorgegeben:

1. beim Psychiater wichtiger

2. beim Hausarzt wichtiger

3. bei beiden Ärzten gleich wichtig

4. unwichtig.

(b) Im zweiten Teilfragebogen (siehe Kapitel 9.7 im Anhang; Fragebogen Teil 2) wurden die gleichen Fragen in gleicher Reihenfolge verwendet. Hier konnte der Befragte auf einer Skala von 1 (trifft nicht zu) bis 10 (trifft voll zu) ihre derzeitige Betreuungssituation beim Hausarzt bzw. beim Psychiater bewerten.

(3) Der dritte Teil des Fragebogens (siehe Kapitel 9.4 im Anhang; Patientendaten) diente dazu, soziodemographische Daten der Patienten zu erfassen. Dazu wurden folgende Angaben erhoben:

- Geschlecht; Alter; Muttersprache

- Aktueller Familienstand

- Schulabschluss; berufliche Situation; Wohnsituation 
- Größe des Wohnortes

- Alter bei erstmaligem Auftreten der psychischen Beschwerden; erste psychische Behandlung; Anzahl der stationären Aufenthalte

- Psychische Mitbehandlung in den letzten 12 Monaten

- Dauer der Beziehung zum Hausarzt bzw. Nervenarzt in Jahren

- Diagnose aus Patientensicht

- Medikamente

- Teilnahme an einer Selbsthilfegruppe

- Betreuung.

Im gesamten Fragebogen wurden die Worte Schizophrenie und psychiatrische Erkrankung bewusst nicht verwendet, sondern mit dem Begriff seelische Erkrankung umschrieben, um eine Abwehrreaktion der Patienten zu vermeiden. Im einleitenden Teil der Arbeit wurde diese Problematik bereits angesprochen.

\subsection{Durchführung der Untersuchung}

Im Oktober 2003 startete die Studie in der Tagesklinik in Eisenach und kurze Zeit später erfolgte die Kontaktaufnahme zur Ambulanz in Bad Salzungen. Beide Einrichtungen wurden zunächst telefonisch kontaktiert. Nach persönlicher Vorstellung der Studie gestatteten beide Einrichtungen, dass Patienten für die Pilotstudie und später für die Studie befragt werden konnten. Im Zeitraum von Oktober 2003 bis Februar 2004 wurden neun Patienten für die Pilotstudie in einem persönlichen Interview durch die Doktorandin befragt. Für die eigentliche Studie war die Befragung in diesen beiden Einrichtungen bis Juni 2004 abgeschlossen. Die Patienten wurden zunächst über ihre behandelnden Ärzte angesprochen, ob sie bereit wären, an dem Projekt teilzunehmen. Bei Zustimmung erfolgte das Interview in den Räumen der Einrichtung.

Bereits im August 2003 wurde das St. Josef-Hospital in Bad Driburg eingeladen, an der Studie teilzunehmen. Um das Projekt genauer vorzustellen, fuhr die Arbeitsgruppe, bestehend aus einem Soziologen, einer Psychologin und der Doktorandin (Medizinerin) am 15. Oktober 2003 nach Bad Driburg. Dort vereinbarte man, gemeinsam die Befragung im März 2004 durchzuführen. Die Behindertenwerkstatt in Bad Driburg wurde daraufhin ebenfalls mit der Bitte angeschrieben, die Datenerhebung in dem oben genannten Zeitraum zu ermöglichen, da viele Patienten, die in der Klinikambu- 
lanz behandelt werden, in der Werkstatt arbeiten. Die Patienten wurden entweder durch behandelnde Ärzte, Sozialarbeiter oder die Doktorandin zu der Studie eingeladen. Die Befragung fand in den Räumen der jeweiligen Einrichtung durch die Doktorandin statt.

Für die Studie im Raum Eisenach wurden die dortigen Fachärzte für Allgemeinmedizin und Fachärzte für Neurologie und Psychiatrie schriftlich um Teilnahme gebeten (siehe Kapitel 9.1 im Anhang; Arztanschreiben). Ihre Adressen wurden über das Ärzteverzeichnis der zuständigen kassenärztlichen Vereinigung ermittelt, ggf. ergänzt über die Gelben Seiten des Telefonbuches von Eisenach und die Gelben Seiten der Deutschen Telekom im Internet.

Vom 03.02.2004 bis 26.02.2004 wurden die angeschriebenen Ärzte von der Doktorandin telefonisch angefragt, ob sie an der Studie teilnehmen wollen. Bei Interesse wurde ein Termin für ein persönliches Gespräch vereinbart, in dem der Ablauf und das Ziel der Studie näher erläutert und den Ärzten Informationen für die Patienten (siehe Kapitel 9.2 im Anhang; Patientenanschreiben) überreicht wurden.

Mit den Nervenärzten wurde vereinbart, jeweils 20 Patienten anzuschreiben (Einschlusskriterien: Volljährigkeit, Vorliegen einer Schizophrenie; nach ICD Klassifikation F20.1-6 und derzeit nicht im akuten Schub). Es sollten Patienten unterschiedlichen Alters und unterschiedlichen Schweregrads der Erkrankung ausgesucht werden, um eine möglichst heterogene Stichprobe zu erhalten. Eine weitere Bitte an die Ärzte war, die entsprechenden Patienten beim nächsten Arztbesuch auf das Anschreiben anzusprechen und eventuelle Fragen mit dem Patienten dazu gemeinsam zu klären. Zeigten die Patienten Interesse an der Teilnahme, sollten sie einen beigefügten Rückantwortumschlag an die Abeilung Allgemeinmedizin in Göttingen senden.

Bei den Hausärzten wurde in ähnlicher Weise verfahren. Sie wurden durch die Doktorandin aufgesucht, über die Studie informiert und gebeten, alle ihre Patienten mit der Diagnose Schizophrenie schriftlich zu informieren. Auch hier sollten die potentiellen Teilnehmer der Befragung ihre positive Rückantwort an die Abteilung Allgemeinmedizin der Universität Göttingen senden.

Nach Eingang der Rückantwort in der Abteilung wurde telefonisch ein Termin durch die Doktorandin für eine persönliche Befragung in der Wohnung des Betroffenen vereinbart. Diese Befragungen fanden von März 2004 bis September 2004 statt. In diesem Zeitraum befragte die Doktorandin ebenfalls Beschäftige der drei Behindertenwerkstätten aus dem Raum Eisenach. Die Kontaktaufnahme zu den drei Werk- 
stätten erfolgte telefonisch im Februar 2004. Nach Vorstellung des Projektes im persönlichen Gespräch erklärten sich die Einrichtungen bereit, die Interviews in Ihren Räumen durchführen zu lassen.

\subsection{Datenanalyse}

Zur statistischen Auswertung wurden die Daten aus den Dokumentationsbögen in das Statistikprogramm SAS, Version 8.1 eingegeben. Die Auswertung beschränkte sich auf Häufigkeitsaufzählungen sowie der Berechnung von Minimum, Maximum, Mittelwert und Standardabweichung bei ausgewählten Variablen.

\subsection{Datenschutz und Genehmigung durch die Ethik-Kommission}

Die Patienten erhielten bei der Einladung zur Studie eine kurze Patienteninformation (siehe Kapitel 9.3 im Anhang; Informed consent) zur näheren Erläuterung des Projektes im Sinne eines „informed consent“. Auf dem Dokumentationsbogen sollten die Patienten ihr Einverständnis geben.

Direkt vor der Befragung wurden die Patienten noch einmal ausführlich von der Doktorandin über das Ziel der Studie informiert, bestehende Fragen geklärt und auf die Anonymität hingewiesen. Gleichzeitig wurde den Patienten zugesichert, zu jeder Zeit der Befragung ihr Einverständnis rückziehen zu können.

Die Studie wurde durch die Ethik-Kommission der Universität Göttingen im Rahmen des BMBF-Förderschwerpunkts „Kompetenznetz Schizophrenie“ (Antragsnummer 15/3/02) genehmigt. 


\section{Ergebnisse}

\subsection{Pilotstudie}

Die Pilotstudie umfasste neun Patienten. Ziel war es, (1) den Dokumentationsbogen, falls möglich, zu kürzen und (2) Fragen, die schwer verständlich für den Patienten waren, zu streichen.

Als Ergebnis der Pilotstudie wurde der Fragebogen um insgesamt 30 Items gekürzt und fokussiert nun deutlicher auf die psychische Erkrankung. Damit Patienten die Thematik der Studie besser erfassen konnten, wurde der Fragebogen um einige offene Fragen gleich zu Beginn des Interviews als „Einstimmung“ ergänzt. Der ursprüngliche Fragebogen sah vor, dass die Betroffenen die Kriterien für die Arztwahl in eine Rangliste bringen sollten. Das fiel mehreren Personen der Pilotstudie zu schwer. Daher wurden nun am Ende der Befragung nochmals offene Fragen angefügt, in denen Patienten zum Ausdruck bringen konnten, was sie sich besonders von der jeweiligen Arztgruppe wünschten.

\subsection{Teilnahme}

Insgesamt wurden 4 Behindertenwerkstätten, 2 Krankenhäuser mit Ambulanzen, 2 Tageskliniken, 5 Neurologen und 23 Fachärzte für Allgemeinmedizin zur Teilnahme an der Studie eingeladen. Die Institutionen sowie die Neurologen waren alle bereit, an der Studie teilzunehmen.

Von den 23 Hausärzten waren 2 trotz mehrmaligen Anrufens nicht erreichbar; 9 (43 \%) gaben positive Rückmeldung. Die anderen Ärzte nannten als Gründe für ihre Nicht-Teilnahme:

- keine schizophrenen Patienten in Behandlung (4 Ärzte)

- keine schizophrenen Patienten in Behandlung, man solle sich an die Neurologen wenden (4 Ärzte)

- die sich in Behandlung befindlichen schizophrenen Patienten seien zu alt und daher für die Befragung ungeeignet (1 Arzt) 
- Mitteilung über Sprechstundenhilfe: „behandelt solche Art von Patienten nicht“ (1 Arzt)

- kein Interesse an der Studie (2 Ärzte).

Insgesamt wurden 227 Patienten um Teilnahme an der Studie gebeten, davon waren 121 zu einem Interview bereit. Allerdings konnten 10 aufgrund der Schwere ihrer Erkrankung nicht am Interview teilnehmen. Tabelle 2 gibt einen Überblick über die Anzahl der Befragten und die Rücklaufquote, bezogen auf die einzelnen Zugangswege.

Tabelle 2: Teilnahmequote von Patienten

\begin{tabular}{|c|c|c|c|c|c|}
\hline \multirow{2}{*}{ Einrichtung } & \multicolumn{5}{|l|}{ Patienten } \\
\hline & Angesprochen & $\begin{array}{l}\text { Teilnahme } \\
\text { Nicht möglich }\end{array}$ & $\begin{array}{c}\text { Netto } \\
\text { Stichprobe } \\
\text { n }\end{array}$ & Teilnahme & $\begin{array}{l}\text { Teilnahme- } \\
\text { quote } \\
\%\end{array}$ \\
\hline Nervenarztpraxen & 77 & - & 77 & 12 & 16 \\
\hline Hausarztpraxen & 28 & 5 & 23 & 9 & 32 \\
\hline Station Bad Driburg & 13 & 2 & 11 & 8 & 62 \\
\hline Ambulanz Bad Driburg & 5 & - & 5 & 4 & 80 \\
\hline Tagesklinik Bad Driburg & 14 & 1 & 13 & 11 & 79 \\
\hline BhW* Bad Driburg & 38 & - & 38 & 27 & 71 \\
\hline Station Bad Salzungen & 3 & - & 3 & 2 & 67 \\
\hline Ambulanz Bad Salzungen & 10 & . & 10 & 7 & 70 \\
\hline Tagesklinik Eisenach & 5 & - & 5 & 5 & 100 \\
\hline BhW* Stedtfeld & 20 & 1 & 19 & 14 & 70 \\
\hline BhW* Waltershausen & 2 & - & 2 & 2 & 100 \\
\hline BhW* Fambach & 12 & 1 & 11 & 10 & 83 \\
\hline Gesamt & 227 & 10 & 217 & 111 & 49 \\
\hline
\end{tabular}

\subsection{Beschreibung der Patienten}

Insgesamt gingen 111 Teilnehmer in die Studie ein, davon 49 (44.1\%) Männer. Überwiegend hatten die Patienten einen Hauptschulabschluss (45 \%) und waren ledig (57,3 \%). Fast ein Drittel der Befragten lebte in eigener Partnerschaft bzw. Familie und über zwei Drittel der Betroffenen waren Rentner (Tabelle 3). 
Tabelle 3: Stichprobenbeschreibung

\begin{tabular}{|c|c|c|}
\hline Merkmal & $\mathbf{n}$ & $\%$ \\
\hline Frauen & 62 & 55.9 \\
\hline Männer & 49 & 44.1 \\
\hline Alter (M;SD) & 43 & 10.1 \\
\hline kein Schulabschluss/unbekannt & 5 & 4.5 \\
\hline Hauptschulabschluss & 50 & 45.0 \\
\hline Mittlere Reife & 36 & 32.4 \\
\hline Fach-/Hochschulreife & 20 & 18 \\
\hline Vollzeit-berufstätig seit mehr als 6 Monaten & 3 & 2.7 \\
\hline Teilzeit-berufstätig seit mehr als 6 Monaten & 3 & 2.7 \\
\hline in beschützter Beschäftigung & 15 & 13.5 \\
\hline arbeitslos gemeldet & 11 & 9.9 \\
\hline Erwerbs-/Berufsunfähigkeit/Früh-/Altersrentner & 21 & 18.9 \\
\hline Anderweitig ohne berufliche Tätigkeit & 4 & 3.6 \\
\hline beschützte Beschäftigung und Berentung & 52 & 46.8 \\
\hline unbekannt/unklar & 2 & 1.8 \\
\hline ledig & 63 & 57.3 \\
\hline verheiratet & 25 & 22.7 \\
\hline geschieden & 18 & 16.4 \\
\hline verwitwet & 1 & 0.9 \\
\hline getrennt lebend & 3 & 2.7 \\
\hline feste Partnerschaft & 49 & 44.5 \\
\hline keine Partnerschaft & 61 & 55.5 \\
\hline in eigener Familie/Partnerschaft & 32 & 28.8 \\
\hline alleine/in nicht-therapeutischer Wohngemeinschaft & 29 & 26.1 \\
\hline bei Eltern (Herkunftsfamilie) & 23 & 20.7 \\
\hline therapeutische Wohngemeinschaft & 17 & 15.3 \\
\hline psychische Übergangseinrichtung, Altersheim & 10 & 9.0 \\
\hline weniger als 5.000 Einwohner & 34 & 31.8 \\
\hline $5.000-25.000$ Einwohner & 33 & 30.8 \\
\hline 25.000 - 100.000 Einwohner & 39 & 36.4 \\
\hline mehr als 100.000 Einwohner & 1 & 0.9 \\
\hline
\end{tabular}


Die Patienten waren im Durchschnitt 43 Jahre alt (Minimum: 21 Jahre, Maximum: 65 Jahre).

Im Mittel erkrankten die Patienten mit 27 Jahren. Die Spannbreite des Ersterkrankungsbeginnes erstreckte sich vom 5. Lebensjahr bis zum 54. Lebensjahr. Wie aus Abbildung 1 ersichtlich, zeigten sich bei knapp 40 \% der Patienten bereits die ersten Symptome vor dem 20. Lebensjahr. Nur noch wenige erkrankten nach dem 40. Lebensjahr.

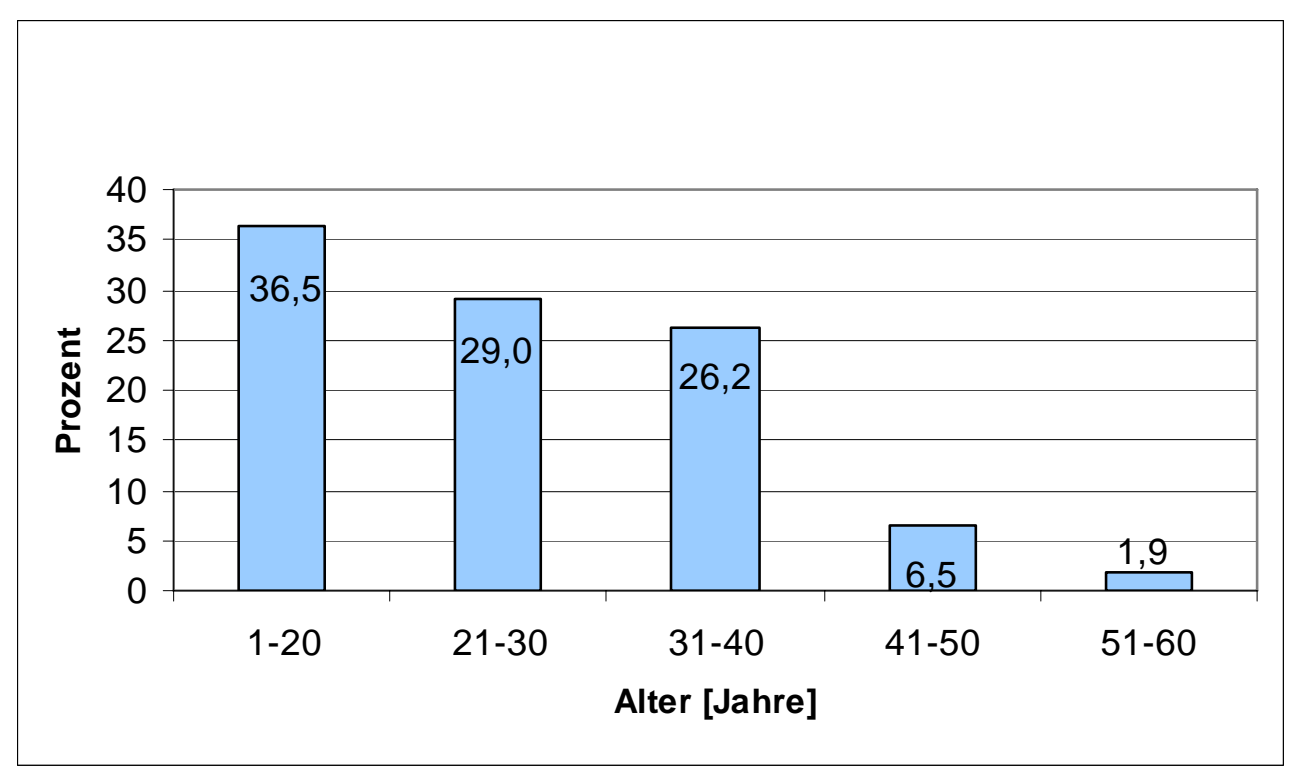

Abbildung 1: Alter zu Beginn der psychischen Erkrankung

Die Mehrzahl der Patienten (65,5 \%) wurde schon vor dem 30. Lebensjahr behandelt. Nur für wenige lag der Zeitpunkt ihrer ersten psychiatrischen Behandlung nach dem 51. Lebensjahr (Abbildung 2). Bei einem Großteil der Patienten lag der Behandlungsbeginn kurz nach Beginn der Erkrankung. 


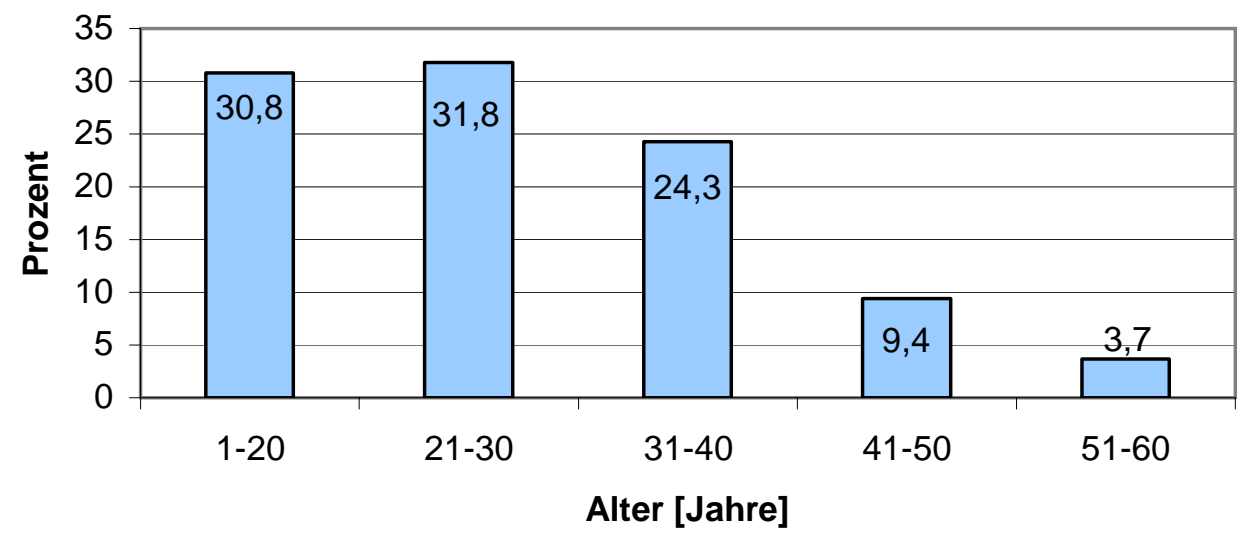

Abbildung 2: Erste psychiatrische Behandlung

Im Durchschnitt wurden die Patienten fünf Mal stationär aufgenommen (Abbildung 3); $83 \%$ hatten ein bis sechs stationäre Aufenthalte. Zwei Patienten waren nie in stationärer Behandlung. Ein Teilnehmer gab an, bereits $35 \mathrm{Mal}$ stationär behandelt worden zu sein.

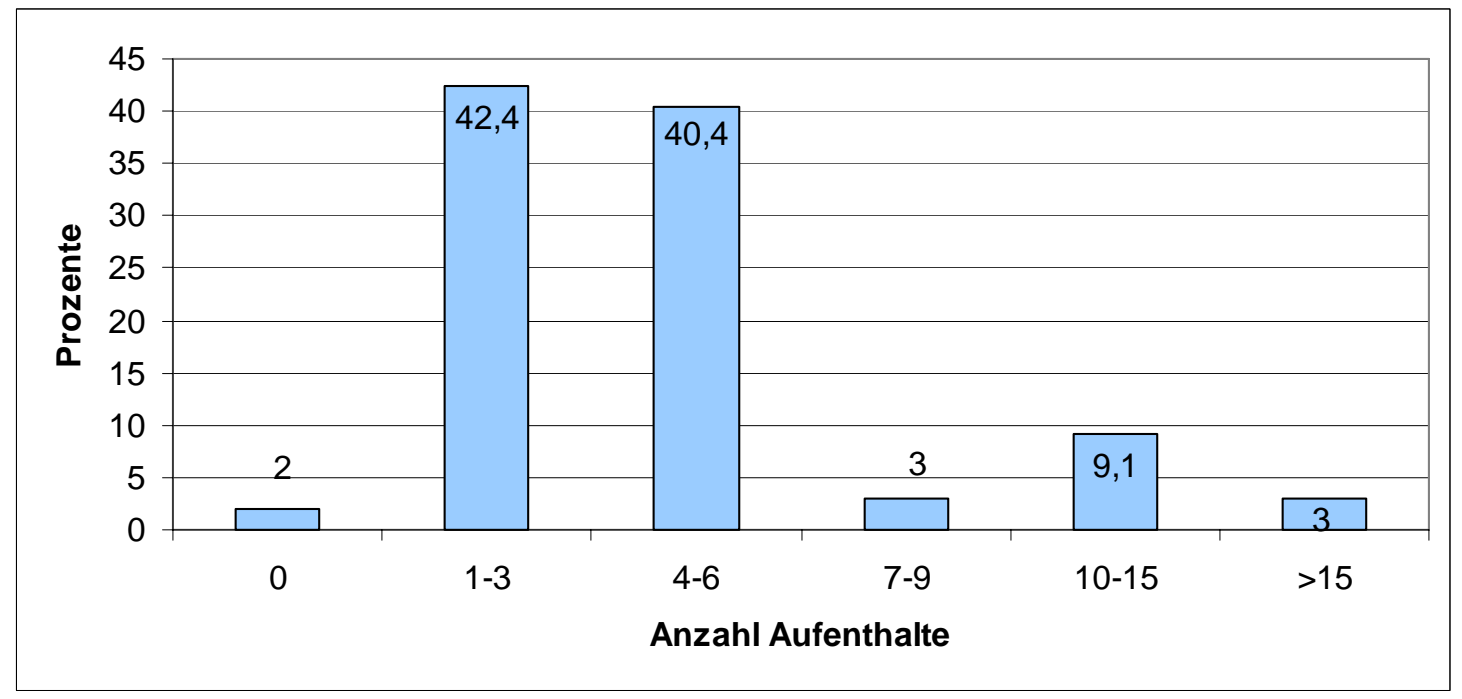

Abbildung 3: Anzahl der stationären Aufenthalte

Im Mittel kannten die Patienten ihren Hausarzt seit 9 Jahren (Minimum: 0.5 Jahre, Maximum: 36 Jahre) und ihren Nervenarzt seit 7 Jahren (Minimum: 1 Jahr, Maximum: 24 Jahre).

Knapp 40 \% (41/106) der befragten Patienten war ein gesetzlicher Betreuer zur Seite gestellt und neun $(8 \%)$ der Studienteilnehmer nahmen aktiv an einer Selbsthilfegruppe teil. 


\subsection{Medikamente}

Von den 111 Patienten nahm ein Befragter keine Medikamente ein; 3 Patienten war der Wirkstoff unbekannt. 47 Befragte gaben an, ein orales Medikament einzunehmen, drei waren alleinig auf ein Depotpräparat eingestellt und 57 nahmen zwei oder mehr Medikamente ein. Am häufigsten wurde ein atypisches Neuroleptikum verordnet (Tabelle 4).

Bei 23 Patienten wurde neben der oralen Medikation ein Depotmedikament verabreicht, wobei in 21 Fällen ein Neuroleptikum verschrieben wurde, in einem Fall ein atypisches Neuroleptikum und ein Patient erhielt ein trizyklisches Antidepressivum.

Tabelle 4: Verordnungshäufigkeit oraler Medikamente

Medikamentenklasse

Atypische Neuroleptika

Neuroleptika

Trizyklische Neuroleptika

Trizyklische Antidepressiva

Anticholinergica

Antidepressiva

Pfl. Antidepressiva

Benzodiazepine

SSRI

Lithium

\section{Anzahl der Patienten}

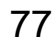

20

4

8

27

4

1

10

3

1

\subsection{Diagnosen}

Die Befragten wurden gebeten, ihre Diagnose bzw. ihre Erkrankung mit eigenen Worten zu benennen; 20 (18\%) Patienten machten hierzu keine Angaben bzw. wussten es nicht. Von denen, die sich zur Diagnose äußerten, nannten sie 41 (45\%) Schizophrenie, 30 \% sprachen von Psychose und $11 \%$ hielten ihre Erkrankung für eine Depression (Tabelle 5). 
Tabelle 5: Selbstgenannte Diagnosen der Patienten

\section{Patienten}

Diagnosen

Schizophrenie

Psychose

Depression

Stoffwechselerkrankung

Neurose

Endogene Psychose aus dem schizophrenen Formenkreis

Paranoide Psychose

Paranoide Schizophrenie

Psychische Erkrankung

Chronische Psychose

Angstzustände

Selbstmordgedanken

Endogene Psychose

Wahnvorstellungen n $\%$

39
43

$27 \quad 30$

10

11

$3 \quad 3$

22

11

11

11

11

11

11

11

11

11

Basis: $\mathrm{n}=91$

\subsection{Versorgungssituation}

In den letzten 12 Monaten vor der Befragung war bei der Mehrzahl der Patienten der niedergelassene Nervenarzt oder der Nervenarzt der Ambulanz der Hauptansprechpartner für psychische Probleme und Medikamente (Abbildung 4). 


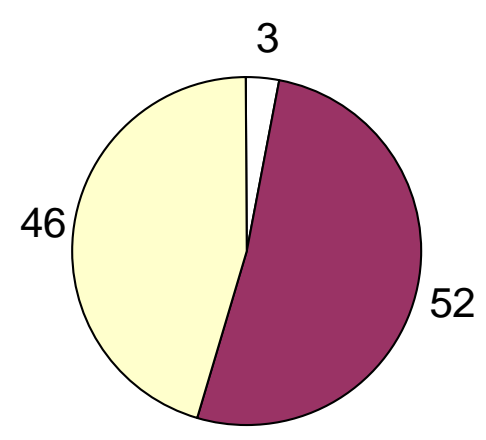

$\square$ Hausarzt

$\square$ Niedergelassene Nervenarzt

$\square$ Ambulanzarzt

Angaben in \%

Abbildung 4: Hauptansprechpartner der Patienten für psychische Probleme und Medikamente

Ein Großteil der Patienten suchte ihren Hausarzt nur wegen körperlicher Beschwerden auf, 25 Teilnehmer gingen wegen körperlicher und manchmal wegen seelischer Beschwerden zum Hausarzt, und nur ein geringer Anteil wendete sich primär wegen seelischer Beschwerden an den Hausarzt. Acht Befragte ließen sich bei NichtErreichen des Nervenarztes Psychopharmaka vom Hausarzt verschreiben (Tabelle $6)$.

Der Nervenarzt wurde von 64 (58 \%) Patienten wegen Medikamenten aufgesucht, von 46 (41\%), wenn es ihnen psychisch schlechter ging (Tabelle 6).

Tabelle 6: Gründe für die Konsultation des Hausarztes bzw. des Nervenarztes

\section{Grund für Konsultation von Hausarzt}

bzw. Nervenarzt

\section{Hausarzt} hauptsächlich seelische Beschwerden körperliche/seelische Beschwerden Überweisung

Blutabnahme

Medikamente

Medikamente

Psychische Verschlechterung

Gespräch
Nur körperliche Beschwerden

Nervenarzt

\section{Patienten}

n

$\%$

77

69

3

3

25

22

21

19

7

6

8

7

Mehrfachantworten waren möglich
64 58

46

41

16 
Auf die offene Frage nach Gründen für dieses Verhalten gab fast die Hälfte der Patienten (41,5\%) an, der Nervenarzt sei der „Experte für seelische Erkrankungen“ und „es sei ja sein Beruf“. Bei 27 (24,3 \%) Befragten hat sich der Kontakt zum Nervenarzt „halt so ergeben“, entweder durch einen stationären Aufenthalt oder durch Verwandte. Vier (3,6 \%) Befragungsteilnehmer gaben als Grund für die Inanspruchnahme des Nervenarztes die Verordnung von Medikamenten an.

Vierzehn (12,6\%) Patienten zweifelten die fachliche Kompetenz des Hausarztes an, vier (3,6 \%) kritisierten, dass ihr Hausarzt keine Medikamente verordne, weitere vier $(3,6 \%)$ Teilnehmer meinten, der Hausarzt habe keine Zeit, seelische Probleme zu behandeln. Neun (8,1 \%) Patienten berichteten, sie seien vom Hausarzt zum Nervenarzt überwiesen worden, da dieser nicht mehr weiter wusste. Fünf (4,5\%) nannten andere Gründe für den Vorzug des Nervenarztes. Drei (2,6 \%) Patienten hielten ihren Hausarzt für fachlich kompetent bei der Behandlung ihrer psychischen Erkrankung, fünf $(4,5 \%)$ nannten Vertrauen als Grund für das Aufsuchen des Hausarztes und fünf (4,5\%) Patienten äußerten andere Gründe.

Im Folgenden sind einige Aussagen von Patienten zu ihrem Hausarzt bzw. Facharzt wiedergegeben:

- „Fühle mich besser beim Nervenarzt aufgehoben“

- Weil sich der Facharzt besser auskennt“

- "Ist Beruf des Arztes“

- „Experte, kennt sich besser aus“

- $\quad$ "Nervenarzt ist halt der Facharzt“

- "Facharzt ist kompetenter"

- Liegt in der Zuständigkeit des jeweiligen Arztes“

- „Nervenarzt ist der Facharzt für psychische Erkrankungen. Er kennt sich mit den Medikamenten für psychische Erkrankungen aus"

- „Fühle mich besser beim Facharzt aufgehoben, dafür ist Ausbildung ja da“

- „Traue das nur dem Facharzt zu“

- „Kenne Nervenarzt vom Krankenhaus“

- „Von Station in Ambulanz“

- Über Mutter zum Facharzt“

- Nervenarzt hat mehr Zeit für umfassende Gespräche“ 
- „Habe mich an Nervenarzt gewöhnt"

- „Nervenarzt kenne ich schon lange, weiß alles über mich“

- „Hausarzt hat mich zum Facharzt überwiesen“

- Will mit Hausarzt nicht darüber reden, bringt nichts“

- „Weiß nicht, ob Hausarzt das könnte“

- „Hausarzt ist für körperliche Dinge zuständig“

- „Glaube, Hausarzt kann Erkrankung nicht einschätzen“

- „Hausarzt kann das nicht“

- $\quad$ „Beim Hausarzt bringt das nichts“

- „ „Fühle mich beim Hausarzt nicht so richtig verstanden, Hausarzt will sich nicht damit befassen, nicht genug Zeit"

- „Beim Hausarzt bleibt keine Zeit für so was“

- _Will Hausarzt nicht damit belästigen“

- $\quad$ „Hausarzt macht das nicht, macht nur das Notwendigste“

- $\quad$ „Muss es nicht jedem erzählen“

- _Würde Hausarzt die Behandlung zutrauen“

- $\quad$ „Denke, Hausarzt kann das managen, nur wenn Krise Facharzt“

- $\quad$ „Werde nur vom Hausarzt betreut, vertraue inm“

- $\quad$ „Beim Hausarzt ist es persönlicher und lockerer. Beim Nervenarzt bin ich angespannt und ich kann nicht so gut über meine Probleme sprechen“

- Es gibt keine Vorzüge dem anderen gegenüber, weil ich zu beiden großes Vertrauen habe und mich bei beiden gleich gut aufgehoben fühle“

- $\quad$ „Hausarzt ist schneller erreichbar als Nervenarzt“

- $\quad$ „Mit Facharzt, das hat sich so ergeben, würde lieber vom Hausarzt betreut werden“

- „ „Früher hat Hausarzt Tabletten aufgeschrieben“

- „Solange ich die Situation noch selber einschätzen kann, gehe ich zum Hausarzt. Ich sage ihm dann, welches Medikament ich denke zu brauchen. Mein Hausarzt weiß nur mangelhaft über meine Erkrankung und meinen Krankheitsverlauf Bescheid, insbesondere weil er erst vor kurzem die hiesige Praxis übernommen hat. (...) Öfter zum Facharzt zu gehen, ist sehr umständlich, da ich eine Wegstrecke von $100 \mathrm{~km}$ auf mich nehmen muss. Ansonsten wäre die Situation eindeutig: Ich würde auf jeden Fall den Facharzt vorziehen". 


\subsection{Wünsche und Bewertung der Patienten}

Zunächst wurden Patienten nach ihren Wünschen und ihren Präferenzen bezüglich Hausarzt bzw. Nervenarzt gefragt. Anschließend konnten sie ihre derzeit behandelnden Ärzte bewerten.

Wie aus Tabelle 7 hervorgeht, wurde die Nähe des Wohnortes, die Praxisatmosphäre, die Länge der Wartezeiten und die Vergabe eines schnellen Termins als besonders wichtig bei beiden Arztgruppen eingeschätzt. Dagegen waren etwa ein Drittel der Patienten die Erreichbarkeit des Arztes und Hausbesuche vergleichsweise unwichtig, insbesondere wenn es den Nervenarzt betraf. Die Vergabe eines schnellen Termins sowie eine angenehme Praxisatmosphäre wurden in der nervenärztlichen Praxis häufiger als in der hausärztlichen gewünscht.

Tabelle 7: Bedeutung der Praxisorganisation - Vergleich der Praxistypen

\begin{tabular}{lcccc} 
& $\begin{array}{c}\text { Bei beiden Ärzten } \\
\text { gleich wichtig }\end{array}$ & $\begin{array}{c}\text { Beim Hausarzt } \\
\text { wichtiger }\end{array}$ & $\begin{array}{c}\text { Beim Facharzt } \\
\text { wichtiger }\end{array}$ & unwichtig \\
\cline { 2 - 5 } Merkmal & $\%$ & $\%$ & $\%$ & $\%$ \\
\hline Praxisatmosphäre $(\mathrm{n}=111)$ & 57 & 6 & 28 & 9 \\
Wartezeiten $(\mathrm{n}=111)$ & 56 & 10 & 10 & 24 \\
Nähe des Wohnortes $(\mathrm{n}=111)$ & 55 & 16 & 18 & 11 \\
Schneller Termin $(\mathrm{n}=111)$ & 50 & 8 & 32 & 10 \\
Erreichbarkeit $(\mathrm{n}=111)$ & 33 & 18 & 14 & 34 \\
Hausbesuche anbieten $(\mathrm{n}=110)$ & 24 & 32 & 6 & 38 \\
& & & &
\end{tabular}

In der Kategorie „Praxisorganisation“ bewerteten die Patienten sowohl ihren Hausarzt als auch ihren Nervenarzt zumeist positiv - mit Mittelwerten zwischen 8 und 9 auf der 10-Punkte-Skala (Tabelle 8). Nur die Aspekte „Wartezeiten“ und „Erreichbarkeit" lagen bei beiden Fachgruppen im Mittelfeld. Ein großer Unterschied von durchschnittlich über 4 Punkten auf der Likert-Skala wurde bei der Bewertung der Frage, „der Arzt macht Hausbesuche“ ersichtlich. Bei den übrigen Themen schnitt zumeist der Hausarzt geringfügig besser als der Nervenarzt ab. Lediglich bei den Fragen nach der „Praxisatmosphäre“ und den „Wartezeiten“ erhielt der Nervenarzt eine etwas bessere Bewertung. 
Tabelle 8: Bewertung der Praxisorganisation - Vergleich der Praxistypen

\begin{tabular}{lcccccc}
\hline & & & \multicolumn{2}{c}{ Trifft voll zu beim } & & \multicolumn{2}{c}{ Trifft nicht zu beim ${ }^{* *}$} \\
Merkmal & Hausarzt & Facharzt & Hausarzt & Facharzt & Hausarzt & Facharzt \\
\cline { 2 - 6 } & $\mathbf{m}^{* * *}$ & $\mathbf{m}^{* * *}$ & $\%$ & $\%$ & $\%$ & $\%$ \\
Praxisatmosphäre & 8.23 & 8.79 & 55 & 67 & 1 & 2 \\
Wartezeiten & 6.27 & 6.87 & 22 & 33 & 6 & 8 \\
Nähe des Wohnortes & 8.77 & 7.91 & 70 & 53 & - & 6 \\
Schneller Termin & 9.00 & 8.22 & 78 & 59 & - & 3 \\
Erreichbarkeit & 5.79 & 5.30 & 30 & 25 & 26 & 30 \\
Hausbesuche anbie- & 8.17 & 4.34 & 66 & 29 & 11 & 51 \\
ten & & & & & & \\
\hline
\end{tabular}

Basis $(N)=111$; einige Fragen wurden z.T. von 1 bis 26 Teilnehmern nicht beantwortet, allerdings wurden 5 der 6 Fragen, die eine Einschätzung des Hausarztes verlangten, von mind. 100 Teilnehmern beantwortet; 4 der 6 Fragen, die eine Einschätzung des Psychiaters abverlangten, wurden von mind. 100 Teilnehmern beantwortet.

* Trifft voll zu: entspricht den Antworten „9“ oder „10“ auf der 10-stufigen Likert-Skala

** Trifft nicht voll zu: entspricht den Antworten „1“ oder „2“ auf der 10-stufigen Likert-Skala

*** m: Mittelwert auf der 10-stufigen Likert-Skala

Fast alle Merkmale der Kategorie „Fachkompetenz“ waren den Patienten entweder bei beiden Ärzten gleich wichtig oder beim Facharzt wichtiger. Eine Ausnahme bildete die „gründliche körperliche Untersuchung“, die 56 \% der Befragten als wichtiger beim Hausarzt bewerteten, ähnlich (wenn auch nicht so eindeutig) fiel das Votum aus, dass der Hausarzt „an andere Ärzte überweist“ (Tabelle 9). Themen, welche die psychische Erkrankung direkt betreffen (z.B. „Erkennen von Frühwarnzeichen“; „Erkennen einer Krankheitsverschlechterung“; „Erklärung der Bedeutung der seelischen Erkrankung für das Leben“; „Anbieten psychotherapeutischer Gespräche“), galten sehr vielen Befragten als wichtiger für die nervenärztliche Praxis. 
Tabelle 9: Bedeutung der Fachkompetenz - Vergleich der Praxistypen

\begin{tabular}{|c|c|c|c|c|}
\hline & $\begin{array}{c}\text { Bei beiden Ärzten } \\
\text { gleich wichtig }\end{array}$ & $\begin{array}{c}\text { Beim Hausarzt } \\
\text { wichtiger }\end{array}$ & $\begin{array}{c}\text { Beim Facharzt } \\
\text { wichtiger }\end{array}$ & unwichtig \\
\hline Merkmal & $\%$ & $\%$ & $\%$ & $\%$ \\
\hline $\begin{array}{l}\text { Beschwerden gleich ernst nehmen } \\
(n=108)\end{array}$ & 79 & 5 & 12 & 4 \\
\hline $\begin{array}{l}\text { Krankheitsverschlechterung rechtzei- } \\
\text { tig erkennen }(n=110)\end{array}$ & 60 & 2 & 34 & 4 \\
\hline Überweisung (n=109) & 56 & 27 & 9 & 8 \\
\hline $\begin{array}{l}\text { Eingehen, wenn keine Medikamen- } \\
\text { teneinnahme erfolgte }(n=92)\end{array}$ & 47 & 2 & 38 & 13 \\
\hline Körperliche Untersuchung ( $n=111)$ & 37 & 56 & 4 & 3 \\
\hline $\begin{array}{l}\text { Wert legen auf Medikamentenein- } \\
\text { nahme }(n=103)\end{array}$ & 33 & 5 & 48 & 14 \\
\hline $\begin{array}{l}\text { Fachwissen bei seelischer Erkran- } \\
\text { kung }(n=109)\end{array}$ & 29 & 1 & 69 & 1 \\
\hline Frühwarnzeichen erkennen $(n=109)$ & 26 & 2 & 57 & 15 \\
\hline $\begin{array}{l}\text { Gespräch über die Bedeutung einer } \\
\text { seelischen Erkrankung für das Leben } \\
(n=109)\end{array}$ & 26 & 1 & 59 & 14 \\
\hline Erfahrung $(n=105)$ & 16 & 2 & 75 & 7 \\
\hline Gespräch üben (n=107) & 13 & 2 & 47 & 38 \\
\hline Psychotherapeut. Gespräch (n=105) & 6 & 1 & 53 & 37 \\
\hline
\end{tabular}

Für die derzeitige Behandlungssituation erhielt der Facharzt fast durchgängig bessere Noten als der Hausarzt, was Mittelwerte bei 8 und 9 in sechs Fragen belegen (Tabelle 10). Wiederum bei den Themen „gründliche körperliche Untersuchung“ und „Überweisung an andere Ärzte“ wurde der Hausarzt besser eingeschätzt. Verhältnismäßig negativ fiel das Urteil über den Hausarzt bei den Fragen aus, die sich näher auf die Behandlung der psychischen Krankheit bezogen. Im mittleren Bereich lagen die Items, „Erkennen von Frühwarnzeichen“, „Anbieten psychotherapeutischer Gespräche“ und „Gespräch über die Bedeutung der seelischen Erkrankung fürs Leben". 
Tabelle 10: Bewertung Fachkompetenz - Vergleich der Praxistypen

\begin{tabular}{|c|c|c|c|c|c|c|}
\hline \multirow[b]{3}{*}{ Merkmal } & \multirow[b]{2}{*}{ Hausarzt } & \multirow[b]{2}{*}{ Facharzt } & \multicolumn{2}{|c|}{$\underline{\text { Trifft voll zu beim }}$} & \multicolumn{2}{|c|}{$\underline{\text { Trifft nicht zu beim }{ }^{\star *}}$} \\
\hline & & & Hausarzt & Facharzt & Hausarzt & Facharzt \\
\hline & $\mathbf{m}^{\star * *}$ & $m^{* * *}$ & $\%$ & $\%$ & $\%$ & $\%$ \\
\hline $\begin{array}{l}\text { Beschwerden gleich ernst } \\
\text { nehmen }\end{array}$ & 8.39 & 9.05 & 67 & 75 & 3 & - \\
\hline $\begin{array}{l}\text { Krankheitsverschlechterung } \\
\text { rechtzeitig erkennen }\end{array}$ & 7.13 & 8.81 & 41 & 71 & 13 & 3 \\
\hline Überweisung & 8.82 & 7.24 & 75 & 57 & 7 & 18 \\
\hline $\begin{array}{l}\text { Eingehen, wenn keine Medi- } \\
\text { kamenteneinnahme erfolgte }\end{array}$ & 6.29 & 8.05 & 37 & 65 & 20 & 11 \\
\hline Körperliche Untersuchung & 8.40 & 5.69 & 66 & 25 & 7 & 26 \\
\hline $\begin{array}{l}\text { Wert legen auf Medikamen- } \\
\text { teneinnahme }\end{array}$ & 6.78 & 9.21 & 36 & 79 & 9 & 1 \\
\hline $\begin{array}{l}\text { Fachwissen bei seelischer Er- } \\
\text { krankung }\end{array}$ & 6.08 & 9.45 & 17 & 87 & 14 & 1 \\
\hline Frühwarnzeichen erkennen & 3.73 & 5.64 & 12 & 32 & 51 & 30 \\
\hline $\begin{array}{l}\text { Gespräch über die Bedeutung } \\
\text { einer seelischen Erkrankung } \\
\text { für das Leben }\end{array}$ & 3.67 & 5.44 & 13 & 32 & 52 & 34 \\
\hline Erfahrung & 6.16 & 9.23 & 20 & 82 & 12 & 1 \\
\hline Gespräch üben & 2.56 & 3.60 & 4 & 14 & 71 & 57 \\
\hline Psychotherapeut. Gespräch & 2.14 & 4.09 & 4 & 25 & 77 & 56 \\
\hline
\end{tabular}

Basis $(N)=111$; einige Fragen wurden z.T. von 1 bis 46 Teilnehmern nicht beantwortet, allerdings wurden 5 der 12 Fragen, die eine Einschätzung des Hausarztes verlangten, von mind. 100 Teilnehmern beantwortet; 6 der 12 Fragen, die eine Einschätzung des Psychiaters abverlangten, wurden von mind. 100 Teilnehmern beantwortet.

* Trifft voll zu: entspricht den Antworten „9“ oder „10“ auf der 10-stufigen Likert-Skala

** Trifft nicht voll zu: entspricht den Antworten „1“ oder „2“ auf der 10-stufigen Likert-Skala

*** m: Mittelwert auf der 10-stufigen Likert-Skala

Wie Tabelle 11 zeigt, sahen die Patienten einen Großteil der Themen in der Kategorie „Arzt-Patienten-Beziehung“ als gleich wichtig für Hausarzt und Facharzt an. Von den Befragten, die sich für eine Fachgruppe speziell festlegten, tendierte die Mehrzahl der Patienten zu der Einschätzung, dass die Aspekte dieser Kategorie für sie beim Facharzt wichtiger sind. Nur der Aspekt „der Arzt kann gut zuhören“ wurde beim Hausarzt als wichtiger eingeschätzt. 
Tabelle 11: Bedeutung der Arzt-Patienten-Beziehung - Vergleich der Praxistypen

\begin{tabular}{|c|c|c|c|c|}
\hline & $\begin{array}{l}\text { Bei beiden Ärzten } \\
\text { gleich wichtig }\end{array}$ & $\begin{array}{l}\text { Beim Hausarzt } \\
\text { wichtiger }\end{array}$ & $\begin{array}{l}\text { Beim Facharzt } \\
\text { wichtiger }\end{array}$ & unwichtig \\
\hline Merkmal & $\%$ & $\%$ & $\%$ & $\%$ \\
\hline Schweigepflicht $(n=111)$ & 81 & 5 & 10 & 4 \\
\hline Respekt erhalten $(n=110)$ & 81 & 4 & 9 & 6 \\
\hline Zuhören können (n=111) & 76 & 20 & 3 & 1 \\
\hline Vertrauen haben $(n=110)$ & 75 & 4 & 18 & 2 \\
\hline Akzeptiert werden $(n=108)$ & 62 & 4 & 26 & 8 \\
\hline $\begin{array}{l}\text { Hineinversetzen in gesundheitliche } \\
\text { Lage }(n=110)\end{array}$ & 59 & 3 & 38 & 18 \\
\hline $\begin{array}{l}\text { Ermuntern, Fragen zu stellen } \\
(\mathrm{n}=109)\end{array}$ & 53 & 6 & 18 & 23 \\
\hline Lange Beziehung ( $n=111)$ & 53 & 5 & 29 & 9 \\
\hline $\begin{array}{l}\text { Gespräch über Probleme erleich- } \\
\text { tern }(n=109)\end{array}$ & 53 & 5 & 37 & 5 \\
\hline $\begin{array}{l}\text { Patienten in Entscheidungen ein- } \\
\text { beziehen }(n=108)\end{array}$ & 50 & 5 & 29 & 16 \\
\hline Viel Zeit haben $(n=111)$ & 50 & 3 & 27 & 9 \\
\hline $\begin{array}{l}\text { Rücksicht auf Vorstellung der Pa- } \\
\text { tienten nehmen }(n=99)\end{array}$ & 37 & 1 & 54 & 8 \\
\hline $\begin{array}{l}\text { Eigene Geschichte erzählen kön- } \\
\text { nen }(n=109)\end{array}$ & 34 & 3 & 37 & 26 \\
\hline Erfragen von Belastungen $(n=111)$ & 32 & 5 & 50 & 13 \\
\hline $\begin{array}{l}\text { Unterstützung der Kontaktpflege } \\
(n=109)\end{array}$ & 32 & 2 & 27 & 39 \\
\hline $\begin{array}{l}\text { Unterstützung der Familienharmo- } \\
\text { nie }(n=107)\end{array}$ & 32 & 4 & 29 & 35 \\
\hline $\begin{array}{l}\text { Vertrauen bei Selbstmordgedan- } \\
\text { ken }(n=109)\end{array}$ & 27 & 3 & 47 & 23 \\
\hline $\begin{array}{l}\text { Über private Probleme reden kön- } \\
\text { nen }(n=111)\end{array}$ & 26 & 4 & 43 & 27 \\
\hline $\begin{array}{l}\text { Gespräch über seelische Erkran- } \\
\text { kung üben }(n=107)\end{array}$ & 13 & 2 & 47 & 23 \\
\hline
\end{tabular}

Auch in dieser Kategorie wurde der Facharzt besser bewertet als der Hausarzt (Tabelle 12). Nur bei den Themen, „lange Arztbekanntschaft“ und „der Arzt begegnet einem mit Respekt" schneidet der Hausarzt etwas besser als der Facharzt ab. Beide Arztgruppen erhielten eine sehr niedrige Bewertung bei der Frage „Gespräch über seelische Erkrankung üben“. Ähnliches fand sich auch bei „Unterstützung der Familienharmonie“ und „Unterstützung der Kontaktpflege“. Etwa ein Drittel der Befragten war hier unzufrieden. Sehr positiv wurden Fragen wie „Zuhören des Arztes“, „Begeg- 
nung mit Respekt“, „Einhalten der Schweigepflicht“, „Vertrauen“ und „Akzeptanz“ bewertet (Mittelwerte um 9).

Tabelle 12: Bewertung der Arzt-Patienten-Beziehung - Vergleich der Praxistypen

\begin{tabular}{|c|c|c|c|c|c|c|}
\hline \multirow[b]{3}{*}{ Merkmal } & \multirow{2}{*}{ Hausarzt } & \multirow[b]{2}{*}{ Facharzt } & \multicolumn{2}{|c|}{ Trifft voll zu beim* } & \multicolumn{2}{|c|}{ Trifft nicht zu beim* } \\
\hline & & & Hausarzt & Facharzt & Hausarzt & Facharzt \\
\hline & $m^{\star \star \star *}$ & $m^{* * *}$ & $\%$ & $\%$ & $\%$ & $\%$ \\
\hline Schweigepflicht & 9.16 & 9.37 & 80 & 83 & 2 & 1 \\
\hline Respekt erhalten & 9.22 & 9.05 & 76 & 74 & - & - \\
\hline Zuhören können & 8.45 & 9.03 & 64 & 73 & 4 & - \\
\hline Vertrauen haben & 8.89 & 9.06 & 75 & 77 & 3 & 2 \\
\hline Akzeptiert werden & 8.56 & 9.06 & 68 & 76 & 4 & 2 \\
\hline $\begin{array}{l}\text { Hineinversetzen in gesund- } \\
\text { heitliche Lage }\end{array}$ & 7.65 & 8.64 & 50 & 71 & 5 & 4 \\
\hline Ermuntern, Fragen zu stellen & 6.28 & 6.85 & 36 & 46 & 21 & 21 \\
\hline Lange Beziehung & 8.20 & 8.19 & 61 & 62 & 5 & 4 \\
\hline $\begin{array}{l}\text { Gespräch über Probleme } \\
\text { erleichtern }\end{array}$ & 7.28 & 8.27 & 46 & 61 & 8 & 3 \\
\hline $\begin{array}{l}\text { Patienten in Entscheidungen } \\
\text { einbeziehen }\end{array}$ & 6.89 & 7.01 & 37 & 49 & 18 & 20 \\
\hline Viel Zeit haben & 7.33 & 7.97 & 48 & 59 & 6 & 4 \\
\hline $\begin{array}{l}\text { Rücksicht auf Vorstellung der } \\
\text { Patienten nehmen }\end{array}$ & 6.21 & 7.55 & 32 & 55 & 22 & 15 \\
\hline $\begin{array}{l}\text { Eigene Geschichte erzählen } \\
\text { können }\end{array}$ & 6.57 & 7.92 & 37 & 57 & 16 & 8 \\
\hline Erfragen von Belastungen & 5.30 & 7.55 & 25 & 55 & 26 & 11 \\
\hline $\begin{array}{l}\text { Unterstützung der Kontakt- } \\
\text { pflege }\end{array}$ & 4.57 & 5.65 & 16 & 33 & 39 & 30 \\
\hline $\begin{array}{l}\text { Unterstützung der Familien- } \\
\text { harmonie }\end{array}$ & 4.44 & 5.77 & 20 & 38 & 45 & 35 \\
\hline $\begin{array}{l}\text { Vertrauen bei Selbstmordge- } \\
\text { danken }\end{array}$ & 6.82 & 8.97 & 48 & 77 & 21 & 3 \\
\hline $\begin{array}{l}\text { Über private Probleme reden } \\
\text { können }\end{array}$ & 6.02 & 7.53 & 33 & 55 & 23 & 14 \\
\hline $\begin{array}{l}\text { Gespräch über seelische Er- } \\
\text { krankung üben }\end{array}$ & 2.56 & 3.60 & 4 & 14 & 71 & 57 \\
\hline
\end{tabular}

Basis $(N)=111$; einige Fragen wurden z.T. von 1 bis 21 Teilnehmern nicht beantwortet, allerdings wurden 13 der 19 Fragen, die eine Einschätzung des Hausarztes verlangten, von mind. 100 Teilnehmern beantwortet; 15 der 19 Fragen, die eine Einschätzung des Psychiaters abverlangten, wurden von mind. 100 Teilnehmern beantwortet.

* Trifft voll zu: entspricht den Antworten „9“ oder „10“ auf der 10-stufigen Likert-Skala

** Trifft nicht voll zu: entspricht den Antworten „1“ oder „2“ auf der 10-stufigen Likert-Skala

*** $\quad \mathrm{m}=$ Mittelwert auf der 10-stufigen Likert-Skala 
Jeweils mehr als $60 \%$ der Befragten wünschten sich sowohl vom Hausarzt als auch vom Nervenarzt, dass man ihnen den „Zweck von Untersuchungen und Behandlungen erklärt“ und ihnen „die Ergebnisse der Untersuchung mitteilt“. „Informationen über die seelische Erkrankung“ und „die Aufklärung über Psychopharmaka“ erwarteten die Befragten häufiger von ihrem Nervenarzt. Für über ein Drittel der Befragten war es eher unwichtig, dass „der Arzt sie auf Selbsthilfegruppen hinweist“, „ihre Angehörigen berät und unterstützt“ und „Maßnahmen zum beruflichen Widereinstieg oder Fortkommen anregt“. Falls Patienten diese Aspekte dennoch wichtig waren, erwartete die Mehrzahl dieses Angebot vom Nervenarzt (Tabelle 13).

Tabelle 13: Bedeutung der Beratung und Information für die Patienten - Vergleich der Praxistypen

\begin{tabular}{|c|c|c|c|c|}
\hline & $\begin{array}{c}\text { Bei beiden Ärzten } \\
\text { gleich wichtig }\end{array}$ & $\begin{array}{c}\text { Beim Hausarzt } \\
\text { wichtiger }\end{array}$ & $\begin{array}{c}\text { Beim Facharzt } \\
\text { wichtiger }\end{array}$ & unwichtig \\
\hline Merkmal & $\%$ & $\%$ & $\%$ & $\%$ \\
\hline Ergebnisse erklären $(n=111)$ & 74 & 12 & 8 & 5 \\
\hline Zweck erklären $(n=110)$ & 61 & 14 & 13 & 12 \\
\hline Aufklärung Medikamente $(n=110)$ & 44 & 4 & 42 & 10 \\
\hline Hinweis Hilfsangebote $(n=107)$ & 41 & 3 & 38 & 18 \\
\hline Angehörige beraten $(n=111)$ & 39 & 5 & 19 & 36 \\
\hline Info seelische Erkrankung $(n=110)$ & 38 & 1 & 53 & 8 \\
\hline Berufl. Fortkommen anregen $(n=107)$ & 35 & 4 & 27 & 34 \\
\hline Hinweis Selbsthilfegruppe $(n=108)$ & 20 & 2 & 30 & 48 \\
\hline
\end{tabular}

Die Bewertung der Patienten zu Information und Beratung durch die beiden Arztgruppen war nicht so positiv wie in den vorangegangen Kategorien. Die Mittelwerte lagen im Durchschnitt zwischen 4 und 7 auf der 10-stufigen Skala. Den höchsten Wert erhielt der Aspekt „Erklärung von Ergebnissen“, wobei der Hausarzt hier etwas besser bewertet wurde als der Nervenarzt. Bei allen übrigen Themen, die inhaltlich im Wesentlichen auf die psychische Erkrankung zugeschnitten waren, wurde der Nervenarzt besser bewertet (Tabelle 14). 
Tabelle 14: Bewertung der Beratung und Information - im Vergleich der Praxistypen

\begin{tabular}{|c|c|c|c|c|c|c|}
\hline \multirow[b]{3}{*}{ Merkmal } & \multirow[b]{2}{*}{ Hausarzt } & \multirow[b]{2}{*}{ Facharzt } & \multicolumn{2}{|c|}{$\underline{\text { Trifft voll zu beim* }}$} & \multicolumn{2}{|c|}{$\underline{\text { Trifft nicht zu beim }{ }^{\star *}}$} \\
\hline & & & Hausarzt & Facharzt & Hausarzt & Facharzt \\
\hline & $\mathbf{m}^{* * *}$ & $m^{* * *}$ & $\%$ & $\%$ & $\%$ & $\%$ \\
\hline Ergebnisse erklären & 8.04 & 7.88 & 61 & 58 & 5 & 7 \\
\hline Zweck erklären & 7.64 & 7.90 & 50 & 55 & 8 & 8 \\
\hline Aufklärung Medikamente & 4.40 & 6.80 & 19 & 45 & 41 & 20 \\
\hline Hinweis Hilfsangebote & 4.60 & 6.81 & 23 & 49 & 42 & 22 \\
\hline Angehörige beraten & 4.19 & 5.66 & 20 & 35 & 50 & 37 \\
\hline Info seelische Erkrankung & 4.05 & 7.42 & 16 & 49 & 44 & 12 \\
\hline $\begin{array}{l}\text { Berufliches Fortkommen anre- } \\
\text { gen }\end{array}$ & 3.49 & 5.31 & 12 & 36 & 59 & 40 \\
\hline Hinweis Selbsthilfegruppe & 2.81 & 4.22 & 10 & 26 & 70 & 53 \\
\hline
\end{tabular}

Basis $(N)=111$; einige Fragen wurden z.T. von 1 bis 16 Teilnehmern nicht beantwortet, allerdings wurden 4 der 8 Fragen, die eine Einschätzung des Hausarztes verlangten, von mind. 100 Teilnehmern beantwortet; 4 der 8 Fragen, die eine Einschätzung des Psychiaters abverlangten, wurden von mind. 100 Teilnehmern beantwortet.

* Trifft voll zu: entspricht den Antworten „9“ oder „10“ auf der 10-stufigen Likert-Skala

** Trifft nicht voll zu: entspricht den Antworten „1“ oder „2“ auf der 10-stufigen Likert-Skala

*** m: Mittelwert auf der 10-stufigen Likert-Skala

\subsection{Bewertung der Arztgruppen und Schulbildung der Befragten}

In Tabelle 15 wird die Bewertung der beiden Arztgruppen unter Berücksichtigung des Bildungsstandes der Patienten dargestellt. Es wurden aus Gründen der Übersicht nur Fragen dargestellt, die sich im Vergleich der Mittelwerte zwischen den 3 Schultypen um mindestens 1.5 Punkte voneinander unterschieden. Von den 88 zu untersuchenden Items war das bei 23 Items der Fall. Um mindestens 1.0 Punkte unterschieden sich 35 der 88 Fragen.

Patienten mit einem Realschulabschluss bewerteten sowohl den Hausarzt als auch den Facharzt in 18 Fragen besser als die übrigen Befragten. Patienten mit Realschulabschluss und Hauptschulabschluss unterschieden sich bei den meisten Fragen nur geringfügig. 
Tabelle 15: Bewertung der Arztgruppen und Bildungsstand der Patienten

\begin{tabular}{|c|c|c|c|}
\hline & $\begin{array}{l}\text { Mittlere Reife } \\
\quad(n=36)\end{array}$ & $\begin{array}{l}\text { Hauptschule } \\
(n=55)\end{array}$ & $\begin{array}{l}\text { Gymnasium } \\
(\mathrm{n}=20)\end{array}$ \\
\hline \multicolumn{4}{|l|}{ Merkmal } \\
\hline & $m^{* * *}$ & $m * * *$ & $m^{* * *}$ \\
\hline Eigene Geschichte erzählen können $(\mathrm{HA})^{\star}$ & 7.3 & 6.7 & 4.7 \\
\hline Hinweis auf Selbsthilfegruppe $(F A)^{\star \star}$ & 5.3 & 4.0 & 2.8 \\
\hline $\begin{array}{l}\text { Krankheitsverschlechterung rechtzeitig erken- } \\
\text { nen }(H A)^{\star}\end{array}$ & 7.8 & 7.3 & 5.4 \\
\hline Hinweis auf Hilfsangebote $(\mathrm{HA})^{\star}$ & 5.9 & 4.1 & 3.5 \\
\hline Erreichbarkeit (FA)** & 5.8 & 5.7 & 2.3 \\
\hline Hinweis auf Hilfsangebote $(F A)^{\star \star}$ & 7.9 & 6.5 & 5.7 \\
\hline Überweisung $(F A)^{\star \star}$ & 7.7 & 7.5 & 5.7 \\
\hline Berufliches Fortkommen anregen $(\mathrm{HA})^{\star}$ & 4.8 & 2.9 & 2.8 \\
\hline Körperliche Untersuchung (FA)** & 6.6 & 5.4 & 4.7 \\
\hline Vertrauen bei Selbstmordgedanken $(\mathrm{HA})^{*}$ & 7.3 & 7.0 & 5.6 \\
\hline Gespräch über seel. Erkrankung üben $(F A)^{\star \star}$ & 4.3 & 3.5 & 2.6 \\
\hline Unterstützung der Kontaktpflege $(\mathrm{HA})^{\star}$ & 5.1 & 4.6 & 3.5 \\
\hline Hinweis auf Selbsthilfegruppe $(\mathrm{HA})^{\star}$ & 3.9 & 2.3 & 2.3 \\
\hline Hineinversetzen in gesundheitl. Lage $(\mathrm{HA})^{\star}$ & 8.3 & 7.5 & 6.8 \\
\hline Ermuntern Fragen zu stellen (HA)* & 6.5 & 6.5 & 5.0 \\
\hline Aufklärung Medikamente (FA) ${ }^{\star \star}$ & 7.8 & 6.0 & 7.2 \\
\hline Berufliches Fortkommen anregen (FA)** & 6.3 & 4.5 & 5.7 \\
\hline Psychotherapeut. Gespräche (FA) ${ }^{\star \star}$ & 5.3 & 3.3 & 3.8 \\
\hline Ermuntern Fragen zu stellen (FA) ${ }^{\star \star}$ & 7.2 & 7.2 & 5.4 \\
\hline Fachwissen bei seel. Erkrankung $(\mathrm{HA})^{\star}$ & 6.3 & 6.4 & 4.8 \\
\hline Hausbesuche anbieten (HA)* & 7.6 & 8.9 & 7.0 \\
\hline Hausbesuche anbieten (FA) ${ }^{\star \star}$ & 4.2 & 5.0 & 3.1 \\
\hline Auskennen mit psych. Störung $(\mathrm{HA})^{\star}$ & 6.3 & 6.4 & 4.8 \\
\hline$H A=$ Hausarzt & & & \\
\hline$F A=$ Facharzt & & & \\
\hline *** m: Mittelwert auf 10-stufiger Likert-Skala & & & \\
\hline
\end{tabular}

\subsection{Bewertung der Arztgruppen und Rekrutierungsort}

Tabelle 16 zeigt einen Vergleich der Mittelwerte der Patienten, die aus ambulanter Behandlung (Hausarzt, Facharzt, Klinikambulanz) rekrutiert wurden, mit Patienten, 
welche in beschützten Werkstätten zur Studie eingeladen wurden. Hier werden nur Fragen dargestellt, die sich in ihren Mittelwerten um mindestens 1.0 Punkte unterschieden (18 Fragen).

In 13 Aspekten zeigten die Patienten, die aus Behindertenwerkstätten rekrutiert wurden, ein höhere Zufriedenheit.

Tabelle 16: Bewertung der Arztgruppen und Rekrutierungsort der Patienten

\begin{tabular}{lcc}
\hline & $\begin{array}{c}\text { Ambulante } \\
\text { Behandlung } \\
(\mathrm{n}=32)\end{array}$ & $\begin{array}{c}\text { Werkstatt } \\
(\mathrm{n}=53)\end{array}$ \\
\cline { 2 - 3 } Merkmal & $\mathbf{m}^{* * *}$ & $\mathbf{m}^{* * *}$ \\
\hline
\end{tabular}

Hinweis auf Hilfsangebote (FA)**

Berufliches Fortkommen anregen (FA)**

Rücksicht auf Vorstellung der Patienten nehmen (HA)*

Psychotherapeut. Gespräche (FA)**

Unterstützung der Familienharmonie (HA)*

Hinweis auf Selbsthilfegruppen (FA)**

Info seelische Erkrankung (HA)*

Hinweis auf Selbsthilfegruppen $(\mathrm{HA})^{*}$

Rücksicht auf Vorstellung der Patienten nehmen (FA)**

Hinweis auf Hilfsangebote (HA)*

Berufliches Fortkommen anregen $(\mathrm{HA})^{*}$

Angehörige beraten $(\mathrm{HA})^{*}$

Frühwarnzeichen erkennen $(\mathrm{HA})^{*}$

Körperliche Untersuchung $(\mathrm{HA})^{*}$

Hineinversetzen in gesundheitliche Lage (FA)**

Viel Zeit haben $(\mathrm{HA})^{*}$

Schneller Termin (HA)*

Vertrauen bei Selbstmordgedanken $(\mathrm{HA})^{*}$

\section{7}

3.5

5.2

2.6

3.6

3.3

3.0

1.5

6.7

3.8

2.3

3.5

2.9

9.5

9.7

8.3

9.7

7.4
7.8

5.4

6.9

4.2

5.1

4.7

4.4

3.7

7.9

5.0

3.5

4.6

3.9

8.1

8.3

7.1

8.7

6.4

\footnotetext{
* $\quad H A=$ Hausarzt

** $\quad F A=$ Facharzt

*** m: Mittelwert auf 10-stufiger Likert-Skala
}

\subsection{Bewertung der Arztgruppen und Geschlecht}

Männer und Frauen bewerteten ihre Ärzte sehr ähnlich. Nur in 5 von 88 Items findet sich eine Mittelwertabweichung um mehr als 1.0 Punkte (Tabelle 17). 
Tabelle 17: Bewertung der Arztgruppen und Geschlecht der Patienten

\begin{tabular}{|c|c|c|c|}
\hline \multirow{2}{*}{\multicolumn{2}{|c|}{ Merkmal }} & $\begin{array}{c}\text { Männer } \\
(\mathrm{n}=49)\end{array}$ & $\begin{array}{c}\text { Frauen } \\
(n=62)\end{array}$ \\
\hline & & $m^{\star * *}$ & $m^{\star * *}$ \\
\hline \multicolumn{2}{|c|}{ Frühwarnzeichen erkennen $(\mathrm{HA})^{\star}$} & 4.5 & 3.1 \\
\hline \multicolumn{2}{|c|}{ Frühwarnzeichen erkennen $(F A)^{\star *}$} & 6.8 & 4.7 \\
\hline \multicolumn{2}{|c|}{ Wartezeiten $(F A)^{\star \star}$} & 7.6 & 6.3 \\
\hline \multicolumn{2}{|c|}{ Hausbesuche anbieten $(\mathrm{HA})^{\star}$} & 7.4 & 8.7 \\
\hline \multicolumn{2}{|c|}{ Berufliches Fortkommen anregen $(\mathrm{HA})^{*}$} & 2.9 & 3.9 \\
\hline * & $\mathrm{HA}=$ Hausarzt & & \\
\hline ** & $F A=$ Facharzt & & \\
\hline & m: Mittelwert auf 10-stufiger Likert-Skala & & \\
\hline
\end{tabular}

\subsection{Rangliste der Patientenerwartung an beide Arztgruppen}

Tabelle 18 zeigt die 10 wichtigsten Merkmale und Verhaltensweisen, die sich die Patienten vom Hausarzt wünschten. Am häufigsten wurde die „körperliche Untersuchung“ und „Hausbesuche“ von den Patienten angegeben sowie Themen, die man in der Kategorie „Praxisorganisation“ einordnen kann.

Tabelle 18: Die 10 wichtigsten Merkmale* des Hausarztes aus Sicht der Patienten

Merkmal

Körperliche Untersuchung ( $\mathrm{n}=111)$

Hausbesuche anbieten $(n=110)$

Überweisung $(n=109)$

Gut zuhören können ( $n=111)$

Erreichbarkeit $(n=111)$

Nähe des Wohnortes $(n=111)$

Zweck von Untersuchungen erklären $(n=110)$

Ergebnisse von Untersuchungen erklären $(n=111)$

Wartezeiten $(n=111)$

Schneller Termin $(n=111)$
$\%$

56

32

27

20

18

16

14

12

10

8

* = Merkmale, die am häufigsten vom Hausarzt gewünscht wurden 
Beim Nervenarzt hatten ganz andere Merkmale für den Patienten Bedeutung (Tabelle 19). Hier standen die „Erfahrung“ und das „Fachwissen bei seelischer Erkrankung" im Vordergrund.

Tabelle 19: Die 10 wichtigsten Merkmale* des Nervenarztes aus Sicht der Patienten

Merkmal

Erfahrung mit psychischer Erkrankung ( $\mathrm{n}=105)$

Fachwissen bei seelischer Erkrankung $(n=109)$

Erklärung Bedeutung seel. Erkrankung fürs Leben $(n=109)$

Erkennen Frühwarnzeichen $(n=109)$

Rücksicht auf eigene Vorstellung nehmen ( $n=99)$

Info über seelische Erkrankung $(n=110)$

Nach Belastungen fragen $(n=111)$

Wert legen auf Medikamenteneinnahme $(n=103)$

Vertrauen bei Selbstmordgedanken $(n=109)$

Gespräch über seelische Erkrankung üben $(n=107)$

\section{$\%$}

75

69

59

57

54

53

50

48

47

47

* = Merkmale, die am häufigsten vom Nervenarzt gewünscht wurden

\subsection{Merkmale mit höchster Patientenbewertung}

Tabelle 20 kann man entnehmen, mit welchen Aspekten die Patienten beim Hausarztbesuch am zufriedensten waren. Weit oben stehen Merkmale, welche die Vertrauensbasis zwischen Arzt und Patient direkt betreffen (z.B. „Begegnung mit Respekt“, „Schweigepflicht einhalten“, „Akzeptanz“). 
Tabelle 20: Merkmale* des Hausarztes mit hoher Patientenbewertung

Merkmal

Begegnung mit Respekt ( $n=105)$

Schweigepflicht einhalten $(n=102)$

9.16

Schneller Termin $(n=103)$

Vertrauen haben $(n=103)$

8.89

Überweisung $(n=99)$

Nähe des Wohnortes $(n=108)$

Akzeptiert werden $(n=101)$

8.56

Gut zuhören können ( $n=107)$

8.45

Körperliche Untersuchung ( $n=102)$

Beschwerden gleich ernst nehmen $(n=102)$
9.00

8.82

8.77

8.40

$m^{* *}$

8.39

* $\mathrm{N}=10$ Merkmale, bei denen der Hausarzt die beste Bewertung erhielt $m^{\star *}=$ Mittelwert auf 10-stufigen Likert-Skala

Auch beim Nervenarzt spielte die Vertrauensbasis zwischen Arzt und Patient eine entscheidende Rolle (Tabelle 21). Außerdem schätzen die Patienten am Nervenarzt sein gutes Fachwissen über die seelische Erkrankung (Mittelwert 9.45). Auch andere Aspekte der Kategorie „Fachkompetenz" wurden durch die Patienten sehr gut bewertet. 
Tabelle 21: Merkmale* des Facharztes mit hoher Patientenbewertung

\section{Merkmal}

Fachwissen bei seelischer Erkrankung $(n=101)$

Schweigepflicht einhalten $(n=102)$

Erfahrung mit psychischer Erkrankung ( $n=96)$

Wert legen auf Medikamenteneinnahme $(n=89)$

Vertrauen haben $(n=103)$

Akzeptiert werden $(n=101)$

Begegnung mit Respekt $(n=105)$

Beschwerden gleich ernst nehmen $(n=102)$

Gut zuhören können ( $n=107)$

Vertrauen bei Selbstmordgedanken $(n=90)$ $\mathbf{m}^{*}$

9.45

9.37

9.23

9.21

9.06

9.06

9.05

9.05

9.03

8.87

${ }^{\star} \mathrm{N}=10$ Merkmale, bei denen der Facharzt die beste Bewertung erhielt $\mathrm{m}^{\star \star}=$ Mittelwert auf 10-stufigen Likert-Skala

\subsection{Persönliche Wünsche der Patienten an die Ärzte}

Zum Abschluss der Befragung erhielten die Patienten die Gelegenheit sich in freier Form dazu zu äußern, was sie sich besonders von ihrem Hausarzt bzw. Nervenarzt wünschen. Die Antworten auf diese offenen Fragen wurden in aussagekräftigen Kategorien zusammengefasst.

\section{Fähigkeiten des Arztes}

Zur Persönlichkeit des Hausarztes äußerten sich insgesamt $30 \%$ der 111 befragten Patienten und $25 \%$ zum Nervenarzt. Deutlich zeigte sich, dass sich die Patienten vor allem einen freundlichen und sympathischen Arzt wünschten. Noch wichtiger schien den Befragten jedoch die Fachkompetenz des Arztes zu sein. Vor allem die Erfahrung des Arztes war entscheidend für die Patienten. Besonders vom Nervenarzt wünschten sie sich entsprechende Erfahrung in der Behandlung des Krankheitsbildes und gutes Fachwissen über die seelische Krankheit (Tabelle 22). 
Tabelle 22: Gewünschte Fähigkeiten des Arztes

\begin{tabular}{|c|c|c|c|c|}
\hline \multirow[b]{2}{*}{ Merkmal } & \multicolumn{2}{|c|}{ beim Hausarzt } & \multicolumn{2}{|c|}{ beim Nervenarzt } \\
\hline & $\mathbf{n}$ & $\%$ & $\mathbf{n}$ & $\%$ \\
\hline Persönlichkeit des Arztes & 33 & 30 & 27 & 25 \\
\hline Ruhe, Geduld & 1 & 3 & 4 & 15 \\
\hline erfahrener Arzt, flexibel & 2 & 7 & 2 & 7 \\
\hline freundlich, nett, höflich, hilfsbereit, umgänglich & 23 & 70 & 17 & 63 \\
\hline einfühlsam, warmherzig, menschlich, väterlich & 7 & 21 & 8 & 30 \\
\hline ehrlich, offen, bestimmend & 4 & 12 & 5 & 19 \\
\hline ordentlich, zuverlässig & 2 & 7 & 2 & 7 \\
\hline Fachkompetenz des Arztes & 46 & 42 & 41 & 37 \\
\hline Erfahrung, Auskennen Krankheit, Überweisung & 17 & 37 & 27 & 66 \\
\hline fachliche Kompetenz, gute Ausbildung & 9 & 20 & 7 & 17 \\
\hline gute Behandlung, Untersuchung & 18 & 39 & 8 & 20 \\
\hline Blutabnahme & 2 & 4 & 1 & 2 \\
\hline Auskennen mit seelischer Erkrankung & 3 & 7 & 1 & 2 \\
\hline Medikamente & 4 & 9 & 9 & 22 \\
\hline Selbsthilfegruppe & - & - & 1 & 2 \\
\hline Nachfragen nach körperlichem Befinden & - & - & 1 & 2 \\
\hline
\end{tabular}

In der fettgedruckten Zeile wird die Prozentzahl aller Patienten genannt, die sich zu dieser Kategorie äußerten. Prozentwerte in den normal gedruckten Zeilen beziehen sich auf die Zahl aller sich zu dieser Kategorie äußernden Patienten (entspricht 100 \%). Mehrfachantworten waren möglich.

\section{Gewünschte Merkmale der Arzt-Patienten-Beziehung}

In der Arzt-Patienten-Beziehung war das Vertrauen sowohl zum Hausarzt als auch zum Nervenarzt der entscheidende Faktor für eine hohe Patientenzufriedenheit. Offene Gespräche waren Patienten offenbar besonders beim Nervenarzt wichtig. Auch die emotionale Unterstützung schien den Patienten besonders am Herzen zu liegen. Die Patienten wünschten sich vor allem, dass der Arzt gut zuhören kann. 
Zum Thema „Beratung und Information“ äußerten sich bedeutend weniger Befragte.

Die meisten äußerten hier den Wunsch, gut beraten zu werden (Tabelle 23).

Tabelle 23: Gewünschte Merkmale der Arzt-Patienten-Beziehung

\begin{tabular}{|c|c|c|c|c|}
\hline \multirow[b]{2}{*}{ Merkmal } & \multicolumn{2}{|c|}{ beim Hausarzt } & \multicolumn{2}{|c|}{ beim Nervenarzt } \\
\hline & $\mathbf{n}$ & $\%$ & $\mathbf{n}$ & $\%$ \\
\hline Vertrauensvollen Verhältnis & 36 & 33 & 55 & 50 \\
\hline Vertrauen & 28 & 78 & 39 & 70 \\
\hline langes Kennen, offenes Gespräch & 9 & 25 & 22 & 40 \\
\hline Schweigepflicht einhalten & 2 & 6 & 2 & 4 \\
\hline Emotionale Unterstützung & 46 & 42 & 42 & 38 \\
\hline Verständnis für Patienten, Verstehen durch Arzt & 13 & 28 & 16 & 38 \\
\hline auf Probleme und Wünsche eingehen & 5 & 11 & 5 & 12 \\
\hline Unterstützung & 2 & 4 & 6 & 14 \\
\hline Zuhören können & 31 & 67 & 22 & 52 \\
\hline Beratung und Information & 18 & 16 & 17 & 15 \\
\hline Ratschläge geben, gute Beratung & 11 & 61 & 11 & 65 \\
\hline Aufklärung, Info über Krankheit, Medikamente & 8 & 44 & 7 & 41 \\
\hline Fragen stellen dürfen & 1 & 6 & 1 & 6 \\
\hline eigene Meinung sagen dürfen & - & - & 1 & 6 \\
\hline
\end{tabular}

In der fettgedruckten Zeile wird die Prozentzahl aller Patienten genannt, die sich zu dieser Kategorie äußerten. Prozentwerte in den normal gedruckten Zeilen beziehen sich auf die Zahl aller sich zu dieser Kategorie äußernden Patienten (entspricht 100 \%). Mehrfachantworten waren möglich.

\section{Rahmenbedingungen und Inhalte der Konsultation}

Zur den Rahmenbedingungen und Inhalte der Konsultation äußerten sich $44 \%$ der Patienten über den Hausarzt und $45 \%$ machten Angaben zum Nervenarzt. Die meisten Befragten wünschten sich, dass der Arzt entsprechend Zeit für sie hat. Das traf sowohl auf den Hausarzt als auch auf den Nervenarzt zu (Tabelle 24). 
Tabelle 24: Gewünschte Rahmenbedingungen und Inhalte der Konsultation

\begin{tabular}{lcccc} 
& \multicolumn{2}{c}{ beim Hausarzt } & \multicolumn{2}{c}{ beim Nervenarzt } \\
\cline { 2 - 5 } Merkmal & $\mathbf{n}$ & $\%$ & $\mathbf{n}$ & $\%$ \\
Rahmenbedingungen, Inhalt Konsultation & 48 & 44 & 49 & 45 \\
Zeit haben und Ruhe & & & & \\
Beratung Angehörige & 25 & 52 & 27 & 55 \\
Wartezeiten & 2 & 4 & 2 & 4 \\
Nähe & 9 & 19 & 3 & 6 \\
Erreichbarkeit, Warten auf Termin & 3 & 6 & 1 & 2 \\
Hausbesuche & 8 & 17 & 10 & 20 \\
Krankschreibung & 1 & 2 & - & - \\
Geräte, technische Ausstattung der Praxis & 2 & 4 & 1 & 2 \\
verständliche Ausdrucksweise & 1 & 2 & - & - \\
& 1 & 2 & 1 & 2 \\
\hline
\end{tabular}

In der fettgedruckten Zeile wird die Prozentzahl aller Patienten genannt, die sich zu dieser Kategorie äußerten. Prozentwerte in den normal gedruckten Zeilen beziehen sich auf die Zahl aller sich zu dieser Kategorie äußernden Patienten (entspricht $100 \%$ ). Mehrfachantworten waren möglich. 


\section{Diskussion}

Die vorliegende Arbeit beruht auf der Methode der standardisierten Befragung. Ziel war es, Kriterien zu bestimmen, nach denen Patienten einen Arzt zur Behandlung und Betreuung im Falle einer Schizophrenie auswählen. Im Mittelpunkt stand die Frage, welche Bedeutung Hausärzte und Psychiater/Neurologen für die Patienten haben, insbesondere in der Langzeitbetreuung. Nur $3 \%$ der 111 befragten Patienten wurden alleinig durch den Hausarzt betreut und $22 \%$ wurden von inm psychiatrisch mitbetreut. Vom Nervenarzt wünschten sich die Patienten vor allem großes Fachwissen und umfangreiche Erfahrung mit der psychiatrischen Erkrankung, vom Hausarzt eine kompetente körperliche Untersuchung und, falls erforderlich Hausbesuche. Besonders positiv wurde bei beiden Arztgruppen die Arzt-Patienten-Beziehung bewertet.

\subsection{Methodik und Erhebungsinstrument}

In der empirischen Sozialwissenschaft ist die Methodik der Befragung die am häufigsten angewandte Methode, mit der ca. 90 \% aller Daten gewonnen werden (Bortz und Döring 2002). Durch eine „regulierte Kommunikation“ sollen reliable und valide Informationen über den Forschungsgegenstand gewonnen werden (Scholl 2003).

Unsere Arbeitsgruppe beschloss, die Daten durch eine mündliche Befragung in Form eines Einzelinterviews mit einem standardisierten Fragebogen zu erheben. Ein wesentlicher Grund hierfür war die Besonderheit des Patientenklientels. Schizophrene Patienten haben häufig kognitive Defizite und zeichnen sich durch ein erhöhtes Misstrauen und soziale Zurückgezogenheit aus (Sielk und Janssen 2004, Wobrock et al. 2004), so dass wir befürchteten, die Patienten bei einer schriftlichen Befragung nicht zu erreichen.

Vorteile beim persönlichen Interview bestehen darin, dass man noch unentschlossene Befragte zur Teilnahme am Interview motivieren kann und gerade bei längeren Befragungen, wie in unserem Fall, die Abbruchwahrscheinlichkeit geringer halten kann (Scholl 2003). Dies hat sich in unserer Arbeit bestätigt. So lag die Teilnahmequote in Einrichtungen, in denen die Patienten direkt durch die Doktorandin zur Studie eingeladen wurden, bei fast 80 \%. Zum anderen ist es möglich, den Patienten Hil- 
festellung bei Unverständnis einzelner Fragen zu geben und die Präsentation visuell mit Hilfe farbiger Antwortkarten zu unterstützen (Scholl 2003).

Nachteile dieses Vorgehens sind der hohe Zeit- und Kostenaufwand, weshalb der Stichprobenumfang notwendigerweise kleiner sein muss. Zum anderen kann die „persönliche Atmosphäre“ im Einzelinterview dazu führen, dass sich Befragte eingeschüchtert fühlen und deshalb ausweichend und eventuell sogar unehrlicher antworten (Bortz und Döring 2002, Scholl 2003). Auch wenn es hierfür keine Hinweise durch entsprechende verbale und non-verbale Signale gab, ist dies in unserer Studie nicht auszuschließen. Scholl (2003) sieht als weitere Nachteile, dass Fragen und Antwortvorgaben vom Interviewer fehlerhaft vorgelesen werden können und der Interviewer sich bewusst falsch verhält, um den Aufwand und die Kosten zu senken.

Unsere standardisierte Befragung sollte zum einen Ergebnisse erhalten, die direkt vergleichbar sind (Priebe et al. 1995), und zum anderen obige Nachteile minimieren - v. a. auch dadurch, dass die Doktorandin vor Beginn der Datenerhebung durch ein 4-wöchiges Praktikum in Bad Driburg die Situation schizophrener Patienten genau kennenlernen konnte.

Eine Schwäche des Fragebogens mit vorformulierten Aussagen besteht darin, dass die Sprache des Patienten nicht immer getroffen werden kann und einzelne Fragen nicht richtig verstanden werden (Klingenberg et al. 1996). Diese zeigte sich bei unserer Erhebung auch bei einigen Fragen, wie z.B. „Der Arzt nimmt Rücksicht auf die eigene Vorstellung“ und „Der Arzt legt Wert auf die Medikamenteneinnahme“. Ein weiterer Nachteil bei vorformulierten Aussagen kann sein, dass einige Aspekte nicht erfasst werden, die für den Patienten besonders relevant sind. Deshalb ergänzten wir den Fragebogen mit 4 offenen Fragen.

Als Befragungsort wurde bewusst ein vertrauter Ort (u.a. Wohnung, Raum der Behindertenwerkstatt) des Patienten gewählt, um eine möglichst natürliche Situation herzustellen und authentische Informationen zu erhalten (Lamnek 2005).

Der Fragebogen selbst wurde von unserer Arbeitsgruppe neu entwickelt, da wir in veröffentlichten Publikationen kein Instrument finden konnten, das uns im vollen Maße für die Befragung geeignet schien. Wichtig war uns ein Instrument, das angemessen auf die psychiatrische Erkrankung zugeschnitten war. Als Grundlage diente uns für die Entwicklung ein bereits standardisierter Fragebogen der „European Task Force for Patient Evaluation of General Practice“, der inzwischen in 14 europäischen Ländern zur Messung der Patientenzufriedenheit im hausärztlichen Bereich einge- 
setzt wurde (Klingenberg et al. 1999). In Anlehnung an den "Qualiskope-A“, einen Fragebogen, der sowohl im hausärztlichen als auch im fachärztlichen Bereich Patientenzufriedenheit misst (Gericke et al. 2004), sortierten wir unsere Fragen in 4 Kategorien („Praxisorganisation“, „Fachkompetenz“, „Arzt-Patienten-Beziehung“ und „Beratung und Information").

\subsection{Untersuchungsorte und Rücklaufquote}

Bei der Stichprobe handelt es sich um eine heterogene Patientengruppe: Patienten aus einer eher ländlichen Region (Bad Driburg) und Patienten einer mittelgroßen Stadt (Eisenach), die aus unterschiedlichen Einrichtungen (Tageskliniken, Ambulanzen, Behindertenwerkstätten, Arztpraxen) kamen.

Von den 21 angeschriebenen Hausärzten (=Praxen) entschlossen sich 9 an der Studie teilzunehmen. Dies entspricht einer Teilnahmebereitschaft von $43 \%$, die im Vergleich zu anderen Studien als befriedigend zu betrachten ist. Bei Aschka et al. (2001) z.B. betrug die Rücklaufquote $48 \%$, bei Kendrick et al. (2000) $43 \%$.

Neun (43 \%) Ärzte begründeten ihre Nichtteilnahme an der Studie damit, dass in ihren Praxisklientel keine schizophrenen Patienten seien. Dies ist verwunderlich. Mehrere Studien in Deutschland (Haasen 1993, Dilling et al. 1978, Weyerer und Dilling 1983, Zintl-Wiegand et al. 1978) gaben die Häufigkeit schizophrener Patienten in der Allgemeinarztpraxis zwischen 0,4 und 0,6 Prozent an. Hier liegt die Vermutung nahe, dass die 9 Ärzte von den 21 angeschriebenen Hausärzten bisher nicht in die Behandlung schizophrener Patienten einbezogen wurden und eventuell gar nicht um die Krankheit ihrer Patienten wissen. Hier bestätigt sich die Aussage von Abholz und Sielk (2003), dass die Behandlung von schizophrenen Patienten für den Hausarzt ein Problem mit geringer Relevanz darstellt. Ein Arzt nannte als Grund für die Nichtteilnahme an der Studie hohes Alter seiner schizophrenen Patienten, 2 Ärzte gaben an, kein Interesse an der Studie zu haben.

Von den 5 angeschriebenen Nervenärzten (=Praxen) waren alle sofort bereit, an der Studie teilzunehmen. Auch die übrigen zur Teilnahme eingeladenen psychiatrischen Einrichtungen wie Tageskliniken, Ambulanzen und Behindertenwerkstätten willigten ohne Ausnahme ein. Das lässt den Schluss zu, dass die in der Arbeit behandelte Thematik im fachärztlichen Bereich als relevanter empfunden wurde. Einige Fachärzte äußerten den Wunsch, über das Ergebnis der Arbeit informiert zu werden, was obige Aussage bekräftigt. 
Insgesamt wurden 227 Patienten zur Studie eingeladen, von denen 10 aufgrund der Schwere der Erkrankung nicht befragt werden konnten und 106 die Teilnahme ablehnten. Damit liegt die Rücklaufquote mit $49 \%$ unterhalb der von vielen vergleichbaren Studien, in denen Patienten zu ihrer Arztzufriedenheit befragt wurden. Bei Dathe (1993) z.B. betrug diese 68,5 \%, bei Jung et al. (2002) 88 \%; Grol et al. (1999) kamen auf eine Rücklaufquote von $55 \%$, Vedsted et al. (2002) auf 86,7 \% und Escarce et al. (2003) auf $74 \%$. Ein Grund für die schlechtere Rücklaufquote in dieser Arbeit ist sicherlich in der Patientengruppe zu suchen. So zeigte sich in Studien, in denen Patienten mit unterschiedlichen psychiatrischen Diagnosen befragt wurden, dass die Rücklaufquote bei schizophrenen Patienten niedriger lag als bei anderen Patienten (Kendrick et al. 2000, Möller-Leimkühler und Dunkel 2003).

Die höchste Akzeptanz fand die Studie in den Einrichtungen (Tagesklinken, Behindertenwerkstätten, Ambulanz), in denen die Doktorandin den Fragebogen den Patienten persönlich vorstellte. Die höchste Ablehnerquote (84 \%) fand sich unter den Patienten, die über Nervenarztpraxen eingeladen wurden. Gründe hierfür könnten einmal die Schwere der Erkrankung sein, die die Teilnahme an der Studie verhinderte bzw. erschwerte. Zum anderen konnten wir nicht prüfen, ob neben der schriftlichen Einladung zur Studie eine zusätzliche mündliche Information von den Nervenärzten an die Patienten ergangen war. Aus Datenschutzgründen oblag es anfangs ausschließlich den Haus- und Nervenärzten, ihre Patienten über die Studie zu informieren. Im hausärztlichen Bereich war die Rücklaufquote mit 36 \% höher, hier wurden allerdings insgesamt weniger Patienten in die Studie einbezogen.

\subsection{Patientenkollektiv}

Die endgültige Stichprobe umfasste 111 Patienten (56 \% Frauen). Das Ersterkrankungsalter (im Mittel 27 Jahre) war vergleichbar mit anderen deutschen Studien, in denen schizophrene Patienten untersucht wurden. So lag der mittlere Erkrankungsbeginn bei Müller et al. (1998), die 364 schizophrene Patienten hinsichtlich ihrer sozialen Situation befragten, bei 27.5 Jahren; ähnlich auch bei Köhn et al. (2004a) mit 82 Studienteilnehmern (Ersterkrankung: 29.8 Jahren) und bei Meyer und Franz (2006) mit 25.2 Jahren. Rössler-Riecher (1997) schätzte in ihrer Übersichtsarbeit den Anteil von Patienten mit einem Erkrankungsbeginn nach dem 40. Lebensjahr zwischen 15 und $25 \%$. In der von uns untersuchten Stichprobe erkrankten nur $8.4 \%$ nach dem 40. Lebensjahr. Damit ist der Anteil der Patienten, bei denen anzunehmen 
ist, dass sich eine stabilere Beziehung zum Hausarzt durch jahrelange Bekanntschaft entwickelt hat, gering.

Andere soziodemographische Daten wie Schulbildung, Familienstand und Wohnungssituation differieren nur wenig von Angaben in der Literatur (an der Heiden et al. 1995, Kallert und Leisse 2000, Müller et al. 1998, Schmid et al. 2006, Meyer und Franz 2006). In unserer Studie lag der Anteil der Patienten, welche berentet und/oder in beschützter Beschäftigung tätig waren, mit $79.5 \%$ deutlich höher als bei anderen Patientenkollektiven. Bei Schmid et al. (2006) waren es $37 \%$ und bei an der Heiden et al. (1995) $36 \%$ der Patienten. Das dürfte vor allem daran gelegen haben, dass 53 Patienten unserer Stichprobe über eine Behindertenwerkstatt rekrutiert wurden.

Obwohl in der medikamentösen Therapie der Schizophrenie derzeit eine antipsychotische Monotherapie favorisiert wird (Messer et al. 2006, Pajonk et al. 2005, Gaebel 2001), nahmen über 50 \% der Studienteilnehmer unserer Stichprobe zwei oder mehr Medikamente ein. Dies deckt sich mit anderen internationalen Studien. So kamen Messer et al. (2006) in ihrer Übersichtsarbeit zur Polypharmazie zu dem Ergebnis, dass rund 40-50 \% der stationär behandlungsbedürftigen schizophrenen Patienten und teilweise bis zu 90 \% der ambulanten Patienten eine antipsychotische Kombinationstherapie erhalten.

Elf Prozent unserer Studienteilnehmer antworteten auf die Frage, an welcher Krankheit sie leiden, mit „Depression“, 18 \% konnten darüber keine Auskunft geben. Da die Rekrutierung der Patienten über Dritte erfolgte und kein Instrument zur Diagnosesicherung angewandt wurde (außer Akteneinsicht in einigen Fällen), lässt es sich nicht absolut sicherstellen, ob es sich bei allen Studienteilnehmern wirklich um schizophrene Patienten handelte.

Allerdings verwendeten in der Studie von Stark und Stolle (1994) Patienten, die erst kurze Zeit an der Krankheit Schizophrenie litten, diesen Begriff typischerweise nicht, um ihre Diagnose zu beschreiben. Vielmehr nannten sie alltagssprachliche Formulierungen, wie krank oder seelisch/psychisch krank. Einige der von Stark und Stolle (1994) befragten Patienten benutzen das Wort Psychose, um ihre Krankheit zu benennen. Auch in der von unserer Arbeitsgruppe untersuchten Stichprobe nannten 31 Patienten den Begriff Psychose. 45 \% der Befragten gaben als Diagnose den Begriff Schizophrenie an, was bei Stark und Stolle (1994) keiner der Patienten tat. Da die Patienten in unserer Studie meist schon viel Jahre an der Erkrankung litten, ist anzu- 
nehmen, dass sie sich inzwischen mit der Krankenrolle identifiziert hatten und den Begriff Schizophrenie nicht mehr scheuten.

\subsection{Versorgungssituation}

Für 97 \% der von uns befragten Patienten war in den letzten 12 Monaten der niedergelassene Nervenarzt oder der Nervenarzt der Ambulanz Hauptansprechpartner für seelische Probleme. Vom Hausarzt wurden in unserer Stichprobe nur 3 (2.5\%) der 111 Patienten primär wegen der Schizophrenie behandelt. Ein Viertel der Studienteilnehmer wurde vom Hausarzt hinsichtlich ihrer psychischen Probleme mitbehandelt; 19 \% suchten den Hausarzt z.B. wegen einer Überweisung zum Nervenarzt auf und $6 \%$ wegen einer Blutentnahme. Der Hauptgrund, weshalb Patienten unserer Studie den Hausarzt aufsuchten, waren bei 69 \% körperliche Beschwerden.

In der Literatur findet man unterschiedliche Angaben zur Inanspruchnahme von Haus- bzw. Nervenärzten durch schizophrene Patienten. Bei Haasen und Stark (1997) waren es 27 \% der Patienten, die eine alleinige Betreuung durch den Hausarzt erhielten; 46 \% suchten regelmäßig den Nervenarzt auf. In einer älteren Studie von Haasen (1993) wurden 31 \% der Schizophreniepatienten allein vom Hausarzt betreut. Zu ähnlichen Ergebnissen kamen auch Bosch und Pietzcker (1975) mit 25 \% und Meller et al. (1990) mit 20.7 \%. Allerdings führten Meller et al. (1990) keine Diagnosespezifizierung durch, so dass hier auch andere Psychoseerkrankungen eingingen. In Studien jüngeren Datums aus Deutschland und dem Ausland fallen die Werte schon etwas niedriger aus. In der Erhebung von Abholz und Sielk (2003) wurden $10 \%$ der Schizophreniepatienten nur durch den Hausarzt betreut. Wie Abholz und Sielk (2003) selbst einschränkend mitteilten, kann man aber davon ausgehen, dass die Anzahl an der Gesamtpopulation noch niedriger ausfällt, weil die wenigen teilnehmenden Praxen ihrer Studie (22 \%) eine Positivauswahl darstellen. Denn die Motivation an einer Umfrage teilzunehmen steigt, wenn man das Problem selbst als relevant erachtet.

In Veröffentlichungen der internationalen Literatur liegt der Anteil der schizophrenen Patienten, die allein vom Hausarzt betreut werden, zwischen 10 und $30 \%$. So waren es in Norwegen 25 \% (Eich 1990), in Australien zwischen 29 \% (Carr et al. 2004) und 31 \% (Lewin und Carr 1998), in den USA 19,3 \% (Yates 1986), in Schottland 7 \% (Lang et al. 1997) und in Irland 22 \% (Gavin et al. 2005). In England haben zwischen $8.5 \%$ und $25 \%$ der Patienten den Kontakt zum psychiatrischen Bereich verloren 
(Freeman und Alpert 1986: 8.5 \%, Freeman et al. 1979: 16 \%, Johnstone et al. 1984: 24 \%, Harvey 1996: 25 \%). Speziell für England muss man berücksichtigen, dass die psychiatrische Basisqualifikation und die entsprechende Fortbildung der Hausärzte eine Hauptaufgabe im englischen Versorgungssystem darstellt (Spieß। und Cording 2000) und der Hausarzt als „Gatekeeper“ fungiert (Bowling und Redfern 2000, Abholz 2006). Dadurch ist die Überweisung zum Facharzt oft überflüssig. So konnte in einigen Studien gezeigt werden, dass zwischen $7 \%$ und $15 \%$ (Prince und Phelan 1994: 7.7 \%, Watts 1973: 15 \%, von Korff et al. 1985: 14 \%) der Schizophrenen nie beim Nervenarzt vorstellig wurden. In einer vergleichsweise neueren Studie empfahlen Prince und Phelan (1994) allerdings, dass Patienten mit Verdacht auf Schizophrenie dem Facharzt zu überweisen wären. Diese „Gatekeeper-Funktion“ des Hausarztes findet man auch in anderen Ländern, wie in den Niederlanden (Verhaak et al. 2004), in Australien und in den Vereinigten Staaten.

Vergleicht man unsere Ergebnisse mit den in der deutschen Literatur veröffentlichten Daten, fällt unter anderem das differente Ergebnis zur Studie von Haasen und Stark (1997) auf. Im Gegensatz zu uns führten Haasen und Stark (1997) ihre Studie an stationären Patienten durch, wovon ein Drittel erstmalig hospitalisiert war. Es ließ sich zeigen, dass die Ersthospitalisierten eher vom Hausarzt betreut wurden. Von diesen hatten vermutlich die meisten noch keinen Kontakt zu einem Nervenarzt. Im weiteren Verlauf der Erkrankung suchten die Betroffenen zunehmend den Nervenarzt auf. An unserer Studie nahmen fast durchweg Langzeiterkrankte teil. In den Studien von Köhn et al. (2004 b) und Fuchs und Steinert (2004) zum Hilfesuchverhalten schizophrener Ersterkrankter in Deutschland suchten ca. 25 \% der Schizophreniepatienten zuerst Hilfe beim Hausarzt. Auch in der Schweiz gingen 25 \% der Betroffenen zuerst zum Hausarzt. Dies waren vor allem Patienten mit unspezifischen Symptomen, während Patienten mit Positivsymptomen meist gleich den Nervenarzt aufsuchten (Platz et al. 2006).

Bei den von Bosch und Pietzcker (1975) und Meller et al. (1990) veröffentlichten Ergebnissen muss man bedenken, dass diese Studien in den frühen 90er Jahren und in den 70er Jahren durchgeführt wurden. Seitdem hat sich das Versorgungssystem für psychisch Kranke verändert, z.B. hat der Anteil an Nervenärzten/Psychiatern zugenommen (Geiselmann und Linden 1989). So waren 1985 nur 34 \% der 7 Millionen psychisch Kranken in der Bundesrepublik Deutschland in nervenärztlicher Behandlung, wogegen $43 \%$ bei Allgemeinärzten und $15 \%$ bei Internisten in Behandlung waren. Bereits 10 Jahre später erbrachten die Nervenärzte $45 \%$ der psychiatrischen Leistungen im Rahmen der kassenärztlichen Versorgung und die 
Leistungen im Rahmen der kassenärztlichen Versorgung und die Hausärzte $47 \%$ (Spieß। und Cording 2000).

Bei Haasen und Stark (1997) suchten 59 \% der schizophrenen Patienten den Hausarzt wegen somatischer Beschwerden auf, nur $22 \%$ gingen zu ihm, wenn sich z.B: ihr psychischer Zustand verschlechterte. Ein Teil der Patienten suchte den Hausarzt auch wegen der Verordnung von Psychopharmaka auf. Bei uns lag dieser Anteil bei $7 \%$, bei Haasen und Stark (1997) bei $15 \%$, bei Nazareth et al. (1995) bei $6 \%$ und bei Kendrick et al. (1994) sogar bei $77 \%$, wobei in der letzteren Studie in der Mehrzahl der Fälle keine Therapieumstellung durch den Hausarzt erfolgte. Sowohl Kendrick et al. (1994) als auch Bindman et al. (1997) erhoben die Daten an Patienten mit unterschiedlichsten psychiatrischen Diagnosen (Schizophrenie, schizoaffektive Störungen, affektive Störungen). Bei Bindman et al. (1997) war ein Fünftel aller Patienten beim Hausarzt in die Überwachung und Behandlung der seelischen Erkrankung einbezogen, die Mehrheit der Patienten (75 \%) suchten den Hausarzt wegen körperlicher Beschwerden auf. In einer Studie von Nazareth et al. (1995) aus England waren ebenfalls körperliche Beschwerden der Hauptgrund, weshalb $40 \%$ der schizophrenen Patienten - It. eigenen Angaben - den Hausarzt aufsuchten; 8 \% kamen hier wegen psychischer Probleme und 6 \% wegen ihren Depotmedikamenten. Nazareth et al. (1995) befragten in ihrer Studie gleichzeitig die Hausärzte über Gründe, weshalb Patienten mit Schizophrenie zu ihnen kämen. Diese Ergebnisse differieren zu den Aussagen der Patienten. So sagten 8 der 21 befragten Hausärzte, die Patienten kämen hauptsächlich mit psychischen Problemen, und nur 5 der Ärzte gaben als Grund für den Besuch der Patienten körperliche Beschwerden an. Eine ähnliche Studie führten Lang et al. (1997) durch. Sie befragten allerdings nur Hausärzte und keine Betroffenen: $6 \%$ der Patienten dieser Ärzte besuchten hauptsächlich wegen seelischer Probleme die Praxis, 19 \% kamen wegen ihrer Depotspritze, $21 \%$ wegen eines medizinischen Attestes und $14 \%$ wegen körperlicher Beschwerden. Am häufigsten war eine Kombination aus mehreren Gründen. Brugha et al. (1989) vermuteten, dass schizophrene Patienten häufig mit körperlichen Beschwerden zum Hausarzt gehen und dadurch die Hausärzte davon abgelenkt werden könnten, den psychischen Zustand zu bewerten.

In unserer Studie wurde vom Hausarzt ca. ein Viertel der Studienteilnehmer hinsichtlich ihrer psychischen Probleme mitbehandelt - ein Ergebnis, das sich auch bei einer Umfrage in der Schweiz (Simon et al. 2005) finden ließ. Insgesamt lässt sich feststellen, dass der Hauptgrund für das Aufsuchen des Hausarztes körperliche Beschwer- 
den sind und sich nur wenige Patienten hinsichtlich ihres psychischen Gesundheitszustandes dem Hausarzt anvertrauen. Vielmehr ist er in die Behandlung mit einbezogen, indem er z.B. Psychopharmaka weiterverordnet und zum Nervenarzt überweist.

Auch Hausärzte fühlen sich meist nur für die körperlichen Beschwerden zuständig und gaben Wissensdefizite in der Schizophreniebehandlung an (Abholz und Sielk 2003, Simon et al. 2005, Lester et al. 2005, Nazareth et al. 1995, Kendrick et al. 1991). In diesen Studien wurde überwiegend der Wunsch der Hausärzte deutlich, rasche Konsil- und Überweisungstermine beim Spezialisten für diese Patienten zu erhalten (Abholz und Sielk 2003, Köhn et al. 2004 b, Verdoux et al. 2005, Prince und Phalan 1994, Carr et al. 2004). So zeigten Carr et al. (2004), die in Australien Hausärzte und „community mental health staff“ befragten, dass sich die Hausärzte in der Therapie und Diagnostik der Schizophrenie weniger zutrauten als die „community mental health staff" und dazu neigten, Patienten mit Schizophrenie zügig zum Spezialisten zu überweisen. Gleichzeitig vermuteten die Hausärzte bei sich Defizite in der medikamentösen Therapie und einen Mangel an klinischer Erfahrung in der Behandlung von psychiatrischen Krankheitsbildern. So sahen die Hausärzte ihre Rolle bei der Behandlung von Schizophreniepatienten eher darin, die Arbeit des Spezialisten zu unterstützen und den gesamten Gesundheitszustand des Patienten zu überwachen. Sie fanden jedoch auch, dass zu ihrem Aufgabengebiet die Früherkennung der Schizophrenie und die Rückfallprophylaxe gehören. Dies wird auch in einigen ausländischen Leitlinien für Allgemeinärzte vorgeschlagen (King und Nazareth 1996, Carr 1997).

In der Literatur wird beschrieben, dass der Hausarzt von einigen Patienten bewusst aufgesucht werde und für diese und deren Familie einen „Vertrauenskredit“ besitze (Eich 1990, Aston et al. 2002). Im Vergleich zum Psychiater kenne der Hausarzt den Patienten seit Jahren und meist auch seine Familie und ist im besten Sinne „Familienarzt" (Geiselmann und Linden 1989, Sielk und Janssen 2004, Eich 1990, Vauth et al. 2004). Auch sei die Hemmschwelle, zum Hausarzt zu gehen, bedeutend niedriger als die, einen Psychiater aufzusuchen (Aston et al. 2002, Meller et al. 1990). Dies bestätigen die Ergebnisse unserer Untersuchung nicht. Zum einen kannten sich $\mathrm{Pa}-$ tienten und Hausarzt bzw. Patienten und Nervenarzt annährend gleich lang (im Mittel 9 bzw. 7 Jahre), der Tatbestand einer langjährigen Hausarztbindung war also nicht überdeutlich ausgeprägt. Zum anderen war der Kontakt zum Hausarzt eher selten und beschränkte sich meist auf körperliche Beschwerden. Es ist anzunehmen, dass 
das frühe Erkrankungsalter der Patienten (im Mittel 27 Jahren) insofern eine Rolle spielte, dass Hausarztbindungen vor Beginn der Erkrankung kaum aufgebaut wurden. Auch Abholz und Sielk (2003) berichten, dass - nach Beobachtung der von ihnen befragten Psychiater - ein großer Teil der Schizophreniepatienten aufgrund des jungen Alters überhaupt keinen Hausarzt haben. Nur knapp $10 \%$ der Patienten unserer Studie erkrankten nach dem 40. Lebensjahr.

In den oben genannten Studien wurden Gründe genannt, weshalb Patienten mit Schizophrenie zum Hausarzt gehen und wie Hausärzte ihre Aufgabe in der Schizophreniebehandlung definieren, aber es wurde selten gefragt, ob Patienten überhaupt eine Behandlung vom Hausarzt hinsichtlich ihrer psychischen Erkrankung erwarten und wie sie ihn in dieser möglichen Rolle einschätzen.

Deutlich zeigte sich in unserer Studie, dass ca. zwei Drittel der befragten Patienten die Themen, die sich direkt auf die psychiatrische Behandlung (z.B. „Bedeutung der seelischen Erkrankung fürs Leben“, „Erkennen von Frühwarnzeichen“, „Wertlegen auf Medikamenteneinnahme“, „Psychotherapeutisches Gespräch erhalten“) bezogen, als wichtiger für den Facharzt werteten. Nur sehr wenigen Patienten waren diese Aspekte alleinig beim Hausarzt wichtig und ca. ein Drittel der Patienten erwartete ein Engagement in diese Richtung von beiden Ärzten.

Fast 70 \% der Patienten war es wichtig, dass der Nervenarzt „viel Fachwissen über die seelische Erkrankung“ besitzt und 75 \% wünschten sich „viel Erfahrung im Hinblick auf die seelische Erkrankung“. Längst nicht so viele Patienten stellten diese Anforderungen an den Hausarzt, was darauf schließen lässt, dass nur ca. 20-30 \% der Schizophreniepatienten eine Mitbehandlung des Hausarztes als wichtig erachteten.

Für King (1992) sind schizophrene Patienten teilweise nicht in der Lage, zwischen Hausarzt und Psychiater zu unterscheiden, was sich in unserer Studie nicht bestätigte. Dies belegen auch die freien Aussagen der Patienten. So sagte fast die Hälfte der Patienten auf die Frage, wieso sie den Nervenarzt vorziehen, „dieser sei der Experte für seelische Erkrankungen“ und es „sei ja sein Beruf“. Nur drei Patienten hielten den Hausarzt für ausreichend kompetent. Vierzehn Patienten zweifelten hingegen die fachliche Kompetenz des Hausarztes an. So sagten einige Patienten, „beim Hausarzt bringt das nichts“ und „fühle mich beim Hausarzt nicht so richtig verstanden“. In einer neuseeländischen Studie mit psychiatrischen Patienten nannten diese ähnliche Gründe für die Ablehnung des Hausarztes. Als Hauptgrund sagten hier 33,8 \%, der 
Hausarzt sei nicht die richtige Person zum Sprechen und 28 \% meinten, man muss psychische Probleme nicht mit allen diskutieren (Bushnell et al. 2005).

Auch in der Bewertung der beiden Arztgruppen wurde der Facharzt bei den Themen, die sich direkt auf die psychiatrische Erkrankung bezogen, besser bewertet.

Ähnlich wie bei den freien Aussagen bewerteten die Patienten unserer Studie den Facharzt bei den Aspekten, die sich direkt auf die psychiatrische Erkrankung bezogen, besser. Gleiches berichten auch Haasen und Stark (1997) von Patienten, die sowohl vom Hausarzt als auch vom Nervenarzt betreut wurden. Hier meinten $68 \%$ der Patienten, der Nervenarzt verstehe psychische Probleme am besten; 79 \% sagten, dass der Nervenarzt die Medikamente festlege und $61 \%$ erhielten ausführliche Gespräche beim Nervenarzt. In einer Studie der Göttinger Arbeitsgruppe im Rahmen des Kompetenznetzes Schizophrenie, in der betroffene Patienten und Angehörige interviewt wurden, sahen Patienten den Hausarzt primär als „Körperarzt“ und den Nervenarzt als „Seelenarzt“, was auch dem psychiatrischen Versorgungssystem in Deutschland entspricht (Himmel et al. 2003). Wenn Menschen an Schizophrenie neu erkranken, suchen nach Köhn et al. (2004 b) und Fuchs und Steinert (2002) 30-40 \% von ihnen gleich einen Psychiater auf. Hier liegt die Vermutung nahe, dass bereits zu Beginn der Erkrankung ein Teil der Patienten die Vorstellung hat, dass der Facharzt für psychische Probleme primär zuständig ist.

In Veröffentlichungen von Geers et al. (1982 und 1984) und Hübner-Liebermann et al. (2005) wurden Psychiater/Neurologen für die poststationäre Behandlung von Schizophreniepatienten favorisiert. Gleiches gilt für die Behandlung im ambulanten Bereich. Wie Abholz und Sielk (2003) formulierten, sind Nervenärzte der Meinung, dass die Behandlung der Schizophreniepatienten generell in die Hand des Spezialisten gehöre und nur in einzelnen Ausnahmefällen der Hausarzt die kontinuierliche Betreuung des Patienten übernehmen solle. Gleiches fand sich auch bei Essex et al. (1990) in England. In unserer Studie äußerten sich nur wenige Patienten in den offenen Fragen zu den Gründen, weshalb sie den Hausarzt vorzogen. Fünf Patienten nannten „Vertrauen“ als Grund für das Aufsuchen des Hausarztes, drei hielten ihn für „fachlich kompetent“ hinsichtlich der psychischen Erkrankung. Einige wenige betonten die „lockere und persönliche Atmosphäre“ beim Hausarzt und die „bessere Erreichbarkeit“. Von keinem der Befragten wurde als Grund die „langjährige Bekanntschaft" oder der Status „Familienarzt" genannt. Auch die beiden Aussagen, welche sich auf die Familie bezogen („Angehörige beraten“ und „Unterstützung der Famili- 
enharmonie“), wurde von über einem Drittel als unwichtig gefunden. Nur $5 \%$ waren diese Aspekte beim Hausarzt wichtiger als beim Facharzt.

Es scheint so, dass bei Langzeiterkrankten die Familienbetreuung teilweise mit durch den Facharzt übernommen wird. Für einen Teil der Patienten scheint diese Thematik keine Bedeutung zu haben, weil enger Kontakt zur Familie fehlt oder evt. auch nicht gewünscht ist. In unserer Stichprobe lebte etwa nur die Hälfte der Patienten in der Herkunftsfamilie oder in Partnerschaft. So konnten auch Jablensky et al. (2000) zeigen, dass nur eine Minderheit psychisch Kranker in eigener Familie lebt; bei Hansson et al. (2002) waren es 20 \%. Daneben lässt sich vermuten, dass für eine Reihe von Patienten unserer Stichprobe der Hausarzt nicht der „Familienarzt“ war. Die Patienten unserer Befragung stammen vorwiegend aus dem städtischen Bereich, wo mehrere Fach- und Hausärzte nebeneinander arbeiten und die Familienbindung zum Arzt geringer sein kann (Köhn et al. 2004 b). Anders könnte es im ländlichen Bereich sein. Hier könnte der Vorteil des Hausarztes, „meist am gleichen Ort wie der Patient zu wohnen, das Leben der Gemeinde und die familiären und lokalen Ressourcen besser zu kennen“ (Eich 1990 S. 228), den Patienten stärker bei der Arztwahl beeinflussen.

Zusammenfassend: Im Gegensatz zu anderen Veröffentlichungen wurde in unserer Studie ein nur geringer Anteil Schizophreniepatienten (3 \%) allein vom Hausarzt wegen ihrer psychischen Erkrankung behandelt, vermutlich bedingt durch die veränderte Versorgungssituation in Deutschland. Bei etwa einem Viertel wurde der Hausarzt mit in die Behandlung einbezogen, ein Ergebnis, was sich auch in anderen Studien finden ließ. Gleichfalls bestätigte sich - wie bereits in anderen Arbeiten herausgearbeitet - dass der Hausarzt von vielen Schizophreniepatienten primär wegen körperlicher Beschwerden aufgesucht wurde. Deutlich zeigte sich in unseren Ergebnissen, dass die Patienten diese Arbeitsteilung zwischen Hausarzt (somatische Beschwerden) und Facharzt (psychische Beschwerden) wünschten und den Facharzt als den Experten für ihre Erkrankung ansahen, da er die entsprechende fachärztliche Ausbildung besitzt.

\subsection{Wünsche der Patienten an die Arztgruppen}

Bei der Auswertung unserer Ergebnisse wurde deutlich, dass typisch hausärztliche Tätigkeiten wie körperliche Untersuchung, Hausbesuche und Überweisung vielen Patienten beim Hausarzt wichtig waren. Beim Nervenarzt waren vor allem die Aspek- 
te von Bedeutung, die sich auf die psychiatrische Erkrankung bezogen. Hier zeigt sich erneut, dass - aus Sicht der Patienten - der Hausarzt für die körperlichen Aspekte zuständig ist und der Nervenarzt "für die Seele“.

Viele Aussagen wurden von Befragungsteilnehmern bei beiden Ärzten als gleich wichtig empfunden. Dies betraf vor allem Aspekte der Arzt-Patienten-Beziehung, wie z.B. Schweigepflicht, Respekt, Zuhören, Vertrauen, Akzeptanz und Verständnis der gesundheitlichen Lage. Die Persönlichkeit des Arztes thematisierten ca. $30 \%$ beim „offenen“ Teil des Interviews. So wünschten sich diese Patienten vor allem einen freundlichen und netten Arzt. Auch die Fachkompetenz des Arztes wurde genannt. Besonders vom Nervenarzt, aber auch vom Hausarzt, wurde erwartet, dass Beschwerden ernst genommen werden, Krankheitsverschlechterung erkannt und die Medikamenteneinnahme überwacht wird. Vom Hausarzt wurde die rechtzeitige Überweisung vorrangig gewünscht, vom Nervenarzt dagegen Fachwissen, Erfahrung mit der psychischen Erkrankung und Erkennen von Frühwarnzeichen. Über 80 \% der Befragten war es wichtig, mit in medizinische Entscheidungen einbezogen zu werden.

Bei den Fragen zur Praxisorganisation ergaben sich keine großen Unterschiede in den Erwartungen der Patienten an den Haus- und Nervenarzt. Über die Hälfte der Befragten waren die Praxisatmosphäre, Wartezeiten, Nähe des Wohnortes und schnelle Terminvergabe bei beiden Arztgruppen gleich wichtig. Dies traf auch für Beratung und Information zu. Hier waren zwei Drittel der Befragten daran interessiert, dass ihnen Ergebnisse und der Zweck der Untersuchungen erklärt wurde, wobei sich kein Unterschied zwischen den Fachgruppen fand. Aufklärung zu Medikamenten, Informationen über die seelische Erkrankung und die Einbeziehung der Angehörigen wurden eher vom Nervenarzt erwartet.

Wir haben nur wenige vergleichbare Studien gefunden, in denen sich Patienten mit chronischen Erkrankungen dazu äußern, was sie sich im Vergleich zwischen Hausarzt und Facharzt von der jeweiligen Arztgruppe wünschen. Hekkink et al. (2003) befragten HIV-Patienten zu ihren Wünschen an Hausarzt, Nervenarzt und das Pflegepersonal. Ihre Erhebung kam zu ähnlichen Ergebnissen wie unsere Studie. Auch hier waren die Aspekte, die sich speziell auf die HIV-Erkrankung bezogen, beim Spezialisten wichtiger.

Für Schizophreniepatienten konnten wir keine vergleichbare Studie finden. Zwar befragten auch Lester et al. (2003) Patienten mit Schizophrenie in einem halbstruktu- 
rierten Interview dazu, was innen bei der hausärztlichen Behandlung besonders wichtig ist, aber sie dehnten diese Frage nicht auf die nervenärztliche Behandlung aus. Bei ihrer Befragung kristallisierte sich heraus, dass u.a. eine empathische Gesprächssituation und die Persönlichkeit des Arztes die Zufriedenheit der Patienten positiv beeinflusse. Außerdem waren Aspekte der Praxisorganisation wie Erreichbarkeit und eine langjährige Arztbekanntschaft den Patienten wichtig. Auch wünschte die Mehrheit der Patienten, mit in ärztliche Entscheidungen einbezogen und ohne Vorurteile „als Freund und nicht als Kind“ behandelt zu werden. Am wichtigsten für die Zufriedenheit mit dem Hausarzt war Patienten in der Studie von Lester et al. (2003) jedoch, dass der Hausarzt innen Mut gibt und die Erkrankung nicht als unheilbar bezeichnet. Dieser Aspekt wurde in unserem Fragebogen nicht berücksichtigt, jedoch nannte auch kein Patient unserer Stichprobe ihn bei den offenen Fragen.

In der Studie von Hamann et al. (2005) wurden stationäre Schizophreniepatienten dazu befragt, inwieweit sie in die medikamentöse Behandlung involviert werden wollen. Auch hier zeigte sich, wie in unserer Erhebung, dass viele Patienten dies wünschten.

Für depressive Patienten rangierten an oberer Stelle die persönlichen Fähigkeiten des Arztes (Symptome erkennen, Zuhören, Informieren), um eine gute Qualität in der Behandlung sicherzustellen (Cooper et al. 2000). Informationen zu erhalten, war auch Patienten anderer Studien mit unterschiedlichsten Diagnosen eines der dringlichsten Anliegen an den Arzt (Schattner et al. 2006, Laine et al. 1996, Klingenberg et al. 1996, Grol et al. 1999, Little et al. 2001, Vedstedt et al. 2002). An erster Stelle der Rangliste bei Cooper et al. (2000) stand Vertrauen in den Arzt. Auch dies war für unsere Patienten besonders wichtig.

Lester et al. (2005) befragten Patienten mit unterschiedlichsten psychiatrischen Diagnosen. Diese wünschten sich vor allem vom Hausarzt empathisches Zuhören, Wissen über die psychiatrische Erkrankung, eine optimistische Grundhaltung, rechtzeitige Überweisung bei Schwierigkeiten und einen kurzfristigen Termin, ohne die Symptome „hochspielen“ zu müssen. Auch wollten die Patienten nach Möglichkeit immer vom gleichen Arzt behandelt werden - ein Aspekt, der auch in anderen Studien häufig genannt wurde (Schattner et al. 2006, Redsell et al. 2007, Infante et al. 2004, Vedstedt et al. 2002). Fragen der Kommunikation, der Information, der Verlässlichkeit, des Vertrauens, der Offenheit und Ehrlichkeit, der Fachkompetenz, der emotionalen Unterstützung und Beratung und des Überweisungsverhaltens spiegelten auch die Wünsche der Patienten anderer Studien wider (Infante et al. 2004, 
Schattner et al. 2004, Little et al. 2001, Grol et al. 1999, Klingenberg et al. 1996, Vedsted et al. 2002, Williams et al. 1995). Groenewegen et al. (2005), welche Daten aus 12 europäischen Ländern untersuchten, konnten zeigen, dass in allen Ländern die Schweigepflicht mit an oberster Position stand. Bereits 1998 werteten Wensing et al. 57 Studien zu Patientenwünschen aus, danach waren Menschlichkeit, Kompetenz/ Genauigkeit und in Entscheidungen einbezogen zu werden, für die Patienten am wichtigsten. Mit Bezug auf die Ergebnisse unserer Studie zu den Patientenwünschen kommt man zu dem Schluss, dass sich Erwartungen von schizophrenen Patienten nicht von denen anderer Patienten unterscheiden.

Die von Klingenberg, Bahrs und Szecseny (1996) definierten typischen hausärztlichen Funktionen, wie Notfallversorgung, Hausbesuche, Beratung und Vermittlung zu anderen Ärzten, wurden auch von den Patienten unserer Studie als typisch vom Hausarzt erwartet. Sie verstehen außerdem unter typischen hausärztlichen Funktionen die persönliche Beziehung, regelmäßige Betreuung und Berücksichtigung der Lebenssituation. Dies erachteten Patienten unserer Stichprobe sowohl beim Hausarzt als auch beim Nervenarzt als wichtig. Ein gutes Vertrauensverhältnis, ausreichend Zeit, Rücksichtnahme auf die eigene Vorstellung, Förderung der Familienharmonie, Unterstützung bei beruflichen Problemen wünschten Patienten häufiger vom Nervenarzt als vom Hausarzt.

Unserem Wissen nach gibt es keine Studien, in denen sich speziell Schizophreniepatienten dazu äußerten, wie sie sich ihren „idealen Nervenarzt“ vorstellen. Björkman et al. (1995) befragten Patienten mit unterschiedlichsten psychiatrischen Diagnosen dazu, was für sie in der ambulanten nervenärztlichen Behandlung wichtig ist. Hier wünschten sich die Patienten vor allem einen Arzt, der sie respektiert, der zuhört und sich für die Patienten interessiert sowie Informationen zu Medikamenten, der Krankheit und alternativen Behandlungsmöglichkeiten. Spießl et al. (2002) fragten psychiatrische Patienten nach ihren Erwartungen an ein psychiatrisches Krankenhaus. Die Patienten nannten hier vor allem eine erfolgreiche Therapie, einfühlsame Ärzte, die Wahrung der Menschenrechte und fachlich kompetente Ärzte. Bei Hansson et al. (1993), die ebenfalls stationäre psychiatrische Patienten befragten, zeichnete sich der Wunsch nach einer guten Arzt-Patienten-Beziehung, Mitentscheidungsrecht und ausreichenden Informationen ab. Die Angaben der Patienten unserer Stichprobe weichen kaum von den oben erwähnten Studienergebnissen ab.

Zusammenfassend: Anhand der zehn wichtigsten Wünsche an den Nerven- bzw. Hausarzt zeigten sich spezifische Erwartungen der von uns befragten Patienten an 
die jeweilige Fachgruppe. So standen beim Hausarzt die typisch hausärztlichen Tätigkeiten bei den Wünschen im Vordergrund, während beim Nervenarzt Erfahrung und Fachwissen zur psychischen Erkrankung und der Umgang mit der Erkrankung und den Patienten Priorität hatten. Vertrauen in den Arzt und patientenorientierte Gespräche hatten bei den Wünschen an den Nervenarzt - im offenen Teil der Befragung - Priorität. Wichtig bei beiden Arztgruppen war den Patienten v.a. eine gute Arzt-Patienten-Beziehung, und dabei besonders die Einhaltung der Schweigepflicht, gegenseitiges Respektieren, Zuhören und Beratung und Information im Allgemeinen. In diesen Punkten unterschieden sich unsere Ergebnisse nicht von denen bereits vorliegender Studien mit anderen Krankheitsbildern.

\subsection{Bewertung der Arztgruppen durch die Patienten}

In der Bewertung der Arztgruppen durch die Patienten spiegeln sich im Wesentlichen die gleichen Tendenzen wider, die sich bereits bei den Wünschen der Patienten zeigten. So erhielten Hausärzte in der Kategorie Praxisorganisation (Nähe des Wohnortes, Hausbesuche und schnelle Terminvergabe) gute Noten. Die Praxisatmosphäre wurde bei beiden Fachgruppen positiv gesehen.

In der Kategorie „Fachkompetenz“ wurde der Hausarzt bei der körperlichen Untersuchung deutlich besser als der Nervenarzt bewertet. Gute Benotungen erhielten beide Fachgruppen zu den Themen „Beschwerden gleich ernst nehmen“, „Erkennen einer Krankheitsverschlechterung“ und „Überwachung der Medikamenteneinnahme“. In den Fragen, die sich auf spezielle Fachkenntnisse bezüglich der psychiatrischen Erkrankung bezogen, erhielt der Nervenarzt auf der Zehn-Punkte-Skala im Mittel drei Punkte mehr als der Hausarzt; aber auch die Einschätzung für den Hausarzt lag noch in einem mittelguten Bereich. Relativ schlechte Noten bekam der Hausarzt bei den wichtigen Themen Erkennen von Frühwarnzeichen und psychotherapeutische Gespräche. Hier schnitten die Nervenärzte zwar besser ab, erreichten aber auch nur mittlere bis ausreichende Noten.

Wesentliche Aspekte der Arzt-Patienten-Beziehung, wie Schweigepflicht, Respekt gegenüber den Patienten, Zuhören, Vertrauen oder Akzeptanz, bewerteten die Patienten unserer Studie als sehr gut, wobei sich keine größeren Unterschiede in der Bewertung von Hausärzten und Nervenärzten fanden. Auch das Einbeziehen in medizinische Entscheidungen, Rücksichtnahme auf eigene Vorstellungen, Fragen der Kommunikation und der Konsultationsdauer wurden für beide Arztgruppen als gut 
eingeschätzt. Bessere Noten erhielt der Nervenarzt dagegen dort, wo es besonders deutlich um die psychische Erkrankung ging: Vertrauen bei Selbstmordgedanken, Hineinversetzen in die gesundheitliche Lage, Fragen nach Belastungen.

Ähnliches ergab sich auch in der Einschätzung der Arztgruppen bezüglich Beratung und Information. Soweit es sich dabei um allgemeine Aspekte handelte, ergaben sich keine größeren Unterschiede zwischen den Fachgruppen und es fielen die Noten relativ gut aus. Im Gegensatz dazu wurden Fragen, die mehr die psychiatrische Erkrankung betrafen, z.B. Informationen über die seelische Erkrankung und Einbeziehung von Angehörigen, beim Nervenarzt besser bewertet. Handlungsbedarf scheint hier in beiden Fachgruppen zu bestehen, da die Noten überwiegend zwischen 2 und 5, also im unteren Bereich der 10-Punkte-Skala, lagen.

Betrachtet man die Literatur zum Thema Patientenzufriedenheit hinsichtlich der ärztlichen Fachkompetenz, finden wir unsere Ergebnisse weitgehend bestätigt. Hekkink et al. (2003) berichteten, dass von seinen befragten HIV-Patienten nur $76 \%$ dem Hausarzt Fachwissen bescheinigten, wogegen alle Patienten den Fachärzten Kompetenz zuschrieben. Gleiches zeigte sich bei Jacobi et al. (2004). Hier waren 27 \% der befragten Rheumapatienten der Meinung, der Hausarzt habe nicht genug Wissen über Rheumaerkrankungen und nur $1 \%$ der Befragten behaupteten dies vom Rheumatologen. Von unseren Befragten waren 87 \% der Meinung, dass der Facharzt umfangreiches Fachwissen zur seelischen Erkrankung hat, wogegen nur 17 \% dies dem Hausarzt bescheinigten. Auch Arthur und Clifford (2004) konnten an Rheumapatienten aufzeigen, dass Patienten, die eine Doppelbetreuung erhielten, den Facharzt höher bewerteten.

In Arbeiten von Van Campen et al. (1998) und Hekkink et al. (2003), die sich mit dem Thema Patientenzufriedenheit im Vergleich Hausarzt/Facharzt befassten, wurde der Hausarzt bei allgemeinen Aspekten - „der Arzt arbeitet effektiv“; „der Arzt kennt meine Probleme sehr gut" und „kümmert sich um eine gute Koordination“ - besser eingeschätzt als der Facharzt. Ging es dagegen um spezifische Probleme der Grundkrankheit, wie Einrichtung der Praxis bei Rheumapatienten (Van Campen et al. 1998), um die Weiterbildung und das Fachwissen, waren die Patienten mit dem Facharzt zufriedener (Hekkink et al. 2003).

Unsere Ergebnisse heben sich im Vergleich dazu in zweifacher Hinsicht hervor: der Nervenarzt erhielt bessere Noten (1) bei Fragen, die ein enges Vertrauensverhältnis zum Arzt erfordern (z.B. „das Besprechen von Selbstmordgedanken“; „private Prob- 
leme“; „soziales Umfeld“) und (2) bei vielen Aspekten der Arzt-Patienten-Beziehung. Letzteres mag an der Spezifität der psychiatrischen Erkrankung liegen.

Gute Bewertungen erhielten beide Arztgruppen in der Kategorie „Arzt-PatientenBeziehung“ bei den Themen Respekt und Schweigepflicht. Auch in anderen Arbeiten bewerteten Patienten die Einhaltung der Schweigepflicht als besonders positiv (Klingenberg et al. 1999, Kerssens et al. 2004, Grol et al. 2000).

Im Folgenden wird auf Themen eingegangen, bei denen Patienten unserer Studie den beiden Arztgruppen nur eine mittelmäßige bis schlechte Bewertung gaben. Das betraf die Erreichbarkeit der Ärzte, die nur von einem Drittel der Patienten als gut empfunden wurde, aber zwei Dritteln wichtig war. Ähnlich sah es bei der Wartezeit aus, die in unserer Studie mit Noten um 6 in beiden Fachgruppen eingeschätzt wurde und damit noch verbesserungsfähig ist. Damit bestätigen sich in unserer Arbeit die Ergebnisse anderer Studien (Grol et al. 2000, Van Campen et al. 1998, Williams and Calnan 1991, Dagdeviren und Akturk 2004, Klingenberg et al. 1999). Auch hier wurde festgestellt, dass lange Wartezeiten und die schlechte Erreichbarkeit der Ärzte häufig von Patienten bemängelt werden und zu Unzufriedenheit führen.

Großer Handlungsbedarf ergibt sich bei Beratung und Information. Eine ausführliche Aufklärung über Medikamente zu erhalten, war $90 \%$ der Patienten wichtig, aber $42 \%$ waren in dieser Frage mit dem Hausarzt unzufrieden und auch beim Nervenarzt signalisierten $20 \%$ der Patienten Verbesserungsbedarf. Gleiches zeigte sich in einer deutschen Studie von Schildmann et al. (2003), die depressive und schizophrene Patienten über die Aufklärung und Einwilligung zur Psychopharmakotherapie befragten. Hier sah die Mehrheit der Patienten die Aufklärung im psychiatrischen Krankenhaus als nicht positiv an, und auch nur die Hälfte der Patienten bewertete die Information über die Therapie durch den Arzt als gut oder sehr gut. Gleiches bestätigten Hekkink et al. (2003) in ihren Ergebnissen. Auch hier waren Patienten mit der Information zu Medikamenten unzufrieden und beklagten sowohl beim Hausarzt als auch beim Spezialisten, dass sie sich nur schwer eine zweite Meinung einholen konnten. Ruggeri et al. (2003), die Schizophreniepatienten aus 5 europäischen Ländern zur Zufriedenheit mit psychiatrischen Einrichtungen befragten, kamen zu dem Ergebnis, dass die Patienten sehr unzufrieden mit den Informationen durch die behandelten Ärzte waren. Gleiches konnte schon Hansson (1989) zeigen, der ambulante Patienten mit unterschiedlichsten psychiatrischen Diagnosen in Schweden zur Zufriedenheit mit dem Nervenarzt befragte. Hier waren $20 \%$ damit unzufrieden, wie sie vom Arzt informiert wurden. Das galt auch für den stationären Bereich (Gigantesco et 
al. 2003, Thapinta et al. 2004, Barker et al. 1996). Nicht richtig vom Arzt informiert zu werden und nicht in Entscheidungen miteinbezogen zu werden, beklagen auch Patienten mit somatischen Krankheitsbildern (Williams und Calnan 1991, Steine et al. 2001, Coulter und Jenkinson 2005).

Die schlechtesten Ergebnisse fanden sich in unserer Studie in der Kategorie „Beratung und Information“ bei den Themen „Angehörige beraten“, „das berufliche Fortkommen anregen“ und „auf die Selbsthilfegruppe hinweisen“. Hier lagen die Noten im hausärztlichen Bereich zwischen 2.8 und 4.1 auf der 10-Punkte-Skala und beim Facharzt zwischen 4.2 und 5.7. Gleiches zeigte sich bei Hansson (1989). Hier waren über $70 \%$ der psychiatrischen Patienten nicht damit zufrieden, wie ihre Angehörigen in die Behandlung miteinbezogen wurden. Die mangelnde Einbeziehung der Angehörigen in Entscheidungsvorgänge und Behandlungsprozesse wurde auch in den Arbeiten von Möller-Leimkühler und Dunkel (2003) und Ruggeri et al. (2003), die psychiatrische Patienten befragten, deutlich. Mängel in der Koordination mit anderen Diensten wurden im fachärztlichen Bereich in der Arbeit von Hekkink et al. (2003) beschrieben.

Ein wichtiger Aspekt in Studien zur Patientenzufriedenheit war die Zeit, die der Arzt den Patienten zur Verfügung stellt. In der Literatur finden sich dazu unterschiedliche Ergebnisse. So waren in der Erhebung von Grol et al. (2000) die Patienten sehr zufrieden mit der zur Verfügung gestellten Zeit, während hingegen bei Williams und Calnan (1991) 25 \% der Befragten meinten, dass der Hausarzt zu wenig Zeit für sie habe. Im stationären Bereich wurden psychiatrische Patienten von Möller-Leimkühler und Dunkel (2003) zur Zufriedenheit befragt. Hier äußerten sich die Patienten negativ zu der zur Verfügung gestellten Zeit in Visite und Einzelgespräch. In unserer Arbeit meinten 48 \% der Patienten, der Hausarzt habe immer genug Zeit für sie, und nur $6 \%$ äußerten sich negativ. Im nervenärztlichen Bereich waren $59 \%$ der Patienten voll zufrieden mit der entgegengebrachten Zeit.

In der Literatur finden sich Hinweise darauf, dass Patienten mit einem höheren Bildungsstand den Arzt meist kritischer bewerten (Klingenberg et al. 1999). Dies bestätigte sich auch bei unserer Befragung. Hier gaben Patienten mit Abitur dem Hausarzt und Facharzt bei einigen Fragen schlechtere Noten als Patienten mit mittlerer Reife oder Hauptschulabschluss. Dies betraf vor allem Themen der Kategorie „Beratung und Information“, wie z.B. „auf Selbsthilfegruppen und Hilfsangebote hinweisen“, und Themen der Arzt-Patienten-Beziehung (z.B. „eigene Geschichte erzählen“, „Vertrauen bei Selbstmordgedanken“). Des Weiteren wird in einigen Untersuchungen darauf 
hingewiesen (Klingenberg et al. 1999, Gruyters und Priebe 1992, Thapinta et al. 2004, Williams und Calnan 1991, Carlson und Aakvik 2006), dass in Abhängigkeit vom Geschlecht die Patientenzufriedenheit variiert. In unserer Erhebung fand sich diesbezüglich kein Hinweis. Wir untersuchten in unserer Arbeit zusätzlich, ob es in der Bewertung in Abhängigkeit vom Rekrutierungsort (ambulante Einrichtung vs. Behindertenwerkstatt) Unterschiede gab. Hier ließ sich feststellen, dass die Patienten, die über eine Behindertenwerkstatt rekrutiert wurden, in einigen Aussagen zufriedener mit den Ärzten waren als Patienten aus ambulanten Einrichtungen. Dies betraf zum einen Themen der sozialen Unterstützung („Hinweis auf Selbsthilfegruppen und Hilfsangebote“, „berufliches Fortkommen anregen“) und zum anderen Themen der Fachkompetenz (,psychotherapeutische Gespräche anbieten“, „Fachwissen bei seelischer Erkrankung").

Zusammenfassend: Wie auch in anderen Studien, wurde der Facharzt in der Frage der Kompetenz und des Fachwissens bezüglich der chronischen Krankheit von unseren Befragten besser als der Hausarzt bewertet. Im Gegensatz zur Literatur wird allerdings auch der Facharzt in vielen Fragen der Arzt-Patienten-Beziehung in unseren Ergebnissen etwas positiver eingeschätzt. Handlungsbedarf zur Verbesserung sahen die Patienten v.a. bei der Wartezeit, Erreichbarkeit und der Beratung und Information zum Krankheitsbild sowie bei der Beratung zu sozialen Fragen. In der Literatur zur Patientenzufriedenheit zeigt sich bei diesen Themen ebenfalls häufig eine nichtoptimale Betreuung. Besondere Erwähnung verdient die relativ negative Einschätzung beider Arztgruppen zum Thema „Erkennen von Frühwarnzeichen“. 


\subsection{Schlussfolgerung}

Die derzeitige Behandlungssituation von an Schizophrenie Langzeiterkrankten wird gegenwärtig von einem Großteil der hier befragten Patienten als positiv erlebt. In den letzten beiden Jahrzehnten hat sich eine Arbeitsteilung zwischen Hausarzt und Nervenarzt entwickelt, die auch von den Patienten anerkannt und gewünscht wird.

Die Rolle des Hausarztes hat sich verändert. Er hat in der alleinigen Behandlung von Schizophreniepatienten eine untergeordnete Rolle. Patienten unterscheiden klar zwischen somatischen Beschwerden, für den sie den Hausarzt als Ansprechpartner sehen, und zwischen psychischen Beschwerden, die vorrangig dem Nervenarzt angetragen werden. Der Nervenarzt identifiziert sich mit der Rolle des zuständigen Arztes und wird von Schizophreniepatienten auch so gesehen. Dennoch übernimmt der Hausarzt bei einem Teil der Patienten weiterhin eine Mitbetreuung bei psychischen Problemen.

Die Befragten sehen derzeit Defizite in der fachlichen Kompetenz für die psychiatrische Behandlung vermehrt im hausärztlichen Bereich. Um die Erkrankung und ein mögliches Rezidiv rechtzeitig zu erkennen und dadurch eine rechtzeitige fachärztliche Intervention einleiten zu können, wäre eine entsprechende Intensivierung der hausärztlichen Weiter- und Fortbildung wünschenswert. Grundkenntnisse in der Therapie (Medikamentenverordnung) wurden ebenfalls vom Hausarzt erwartet und sollten häufiger Gegenstand der Ausbildung sein. Die Häufigkeit und die Bedeutung der Schizophrenie erfordern aber genauso vom Nervenarzt eine ständige Auffrischung des Wissens, besonders im Bereich der Früherkennung der Erkrankung.

Um eine hohe Patientenzufriedenheit zu erreichen und auf diese Weise die Compliance zu verbessern, ist vor allem die Beziehung zum Patienten bei beiden Arztgruppen entscheidend. Entsprechende ärztliche Bemühungen werden von Patienten anerkannt und positiv bewertet. Den Patienten sind Beratung und Information über die Erkrankung und die Verordnung der Medikamente wichtig. Dies wird von beiden Fachgruppen erwartet und könnte sicher noch verbessert werden. Lücken bestehen hier vor allem in der Beratung von Angehörigen und in sozialen Fragen. Denkbar wäre hier z.B. eine engere Zusammenarbeit mit sozialen Diensten. Erreichbarkeit des Arztes und Wartezeiten in der Praxis könnten durch bessere Praxisorganisation verbessert werden. 
Fachwissen und Erfahrung mit der Erkrankung sind die dominierenden Faktoren, weshalb sich die Patienten für die nervenärztliche Behandlung entscheiden. Die Spezifität der Erkrankung erfordert - und fördert - ein besonders hohes Vertrauensverhältnis zum Nervenarzt. Nicht wenige Schizophreniepatienten wünschen daher, dass der Nervenarzt teilweise hausärztliche Funktionen übernimmt.

Abschließend darf man wohl die momentane Betreuungssituation von Schizophreniepatienten als zufriedenstellend bezeichnen, sehr wohl aber kann sie durch die genannten und andere Maßnahmen noch optimiert werden. Der Hausarzt ist in die Erkrankung der Schizophreniebehandlung teilweise miteinbezogen. Es sollte von inm nicht erwartet werden - und es ist auch vom Patienten nicht gewünscht - ,dass er die alleinige Behandlung von Schizophreniepatienten übernimmt. Seine Hauptaufgabe - so sehen es die hier befragten Patienten - sollte weiterhin die Behandlung der somatischen Beschwerden bleiben. Ausreichende Grundkenntnisse in der Schizophrenieerkennung und Behandlung wären dennoch wünschenswert. Hierzu sollten in der Facharztausbildung der Hausärzte verstärkt auch psychiatrische Krankheitsbilder und vor allem Wege der kooperativen Betreuung, wie sie unter dem Schlagwort „shared care" diskutiert werden (von Korff und Tiemens 2000), vermittelt werden. Dazu könnten eine Verlängerung bzw. Intensivierung des Ausbildungsabschnittes „Psychiatrie" sowie regelmäßige Fortbildungen und Publikationen auch in allgemeinmedizinischen Zeitschriften beitragen. Der bereits eingeschlagene Weg in der Fortbildung zur Früherkennung der Schizophrenieerkrankung (Aston et al. 2002, Vauth et al. 2004, Bottlender und Möller 2004, Maurer et al. 2006, Ruhrmann et al. 2003) ist ein gutes Zeichen. 


\section{Zusammenfassung}

Hintergrund: Unterschiedliche und teils nur bedingt voraussehbare Verläufe der Schizophrenie, die oft lebenslang erforderliche Medikation und die Dauerbetreuung der Patienten machen diese Erkrankung für die Betroffenen und die Behandler zu einer ständigen Belastung und Herausforderung. In der Literatur wird die kooperative Betreuung der Patienten durch Haus- und Nervenärzte postuliert. Ziel der Arbeit war es, zu untersuchen, mit welchen Erwartungen Patienten zum Haus- und Facharzt gehen und inwieweit sie mit ihrer derzeitigen Behandlungssituation zufrieden sind.

Methode: Patienten mit Schizophrenie sollten in Form von Einzelinterviews mit einem standardisierten Fragebogen zu den Wünschen an den Haus- und Nervenarzt und zu der Bewertung dieser beiden Arztgruppen befragt werden. Der Fragebogen bestand aus 44 Fragen zu den Wünschen an den Arzt und 44 Fragen, in denen die Befragten ihre derzeitige Betreuung durch Hausarzt und Psychiater bewerten konnten. Vier offene Fragen sollten darüber Auskunft geben, in welchen Situationen die Patienten zu welchem Arzt (Hausarzt/Nervenarzt) gehen, ihre Gründe hierfür und welche Eigenschaften bzw. welches Verhalten Hausärzte und Nervenärzte haben sollten. Die Erhebung fand im Raum Eisenach bzw. Bad Driburg statt. In Eisenach wurden die Patienten über ambulante Haus- und Nervenarztpraxen, über Behindertenwerkstätten, Tageskliniken und Ambulanzen zur Befragung eingeladen, in Bad Driburg über eine Behindertenwerkstatt, eine Tagesklinik und die Ambulanz.

Ergebnisse: Es wurden 111 schizophrene Patienten (Teilnahmequote $49 \%$ ) befragt. Für $97 \%$ der 111 Befragten war der Nervenarzt der Hauptansprechpartner für seelische Probleme, für 3 Personen der Hausarzt und $22 \%$ der Betroffenen ließen sich gelegentlich vom Hausarzt wegen der psychischen Erkrankung mitbehandeln. Als Hauptgrund für die Bevorzugung des Nervenarztes nannten 46 (42 \%) der Betroffenen die fachliche Kompetenz. Im Hausarzt sahen $69 \%$ der Befragten vorwiegend den Arzt für körperliche Probleme. Bei beiden Fachgruppen war den Patienten die Arzt-Patienten-Beziehung besonders wichtig, ebenso das Einhalten der Schweigepflicht und persönlicher Respekt. Die meisten Befragten waren mit beiden Arztgruppen in fast allen Bereichen sehr zufrieden, was überwiegend Noten von 8 und mehr auf einer 10-Punkte-Skala belegten. Tendenziell erhielt der Hausarzt in den für inn typischen Aufgabengebieten wie Hausbesuche und körperliche Untersuchung um 3 Punkte bessere Noten als der Nervenarzt. Der Nervenarzt erhielt dagegen als psy- 
chiatrischer Ansprechpartner eine positivere Bewertung (Notenunterschiede im Durchschnitt ebenfalls um 3 Punkte). Handlungsbedarf zur Verbesserung sahen die Patienten vor allem in der Früherkennung der Erkrankung, in der Erreichbarkeit und bei der Beratung und Information zum Krankheitsbild (Noten zwischen 3 und 6).

Schlussfolgerung: Die in den letzten Jahren entstandene Arbeitsteilung zwischen Hausarzt und Facharzt bei der Betreuung von Schizophreniepatienten wird von den Betroffenen größtenteils positiv bewertet. Der Hausarzt übernimmt vorrangig die Behandlung der körperlichen Beschwerden, ist aber dennoch bei einem Teil der Patienten in die Behandlung der psychiatrischen Erkrankung involviert. Das legt eine Optimierung der hausärztlichen Ausbildung im Bereich der Psychiatrie nahe, z.B. durch Verlängerung des Ausbildungsabschnittes „Psychiatrie“ oder durch patientennahe Fortbildungen. Besonders wichtig sind hierbei aus Sicht der Patienten vor allem die Früherkennung der Erkrankung und das rechtzeitige Erkennen eines Rezidivs sowie eine bessere Informationsgestaltung und Beratung der Patienten in sozialen Fragen. Gerade in diesen letztgenannten Bereichen sahen Patienten auch Handlungsbedarf für die nervenärztliche Betreuung. 


\section{Literaturverzeichnis}

Abholz HH (2006): Warum lassen sich Hausärzte so leicht der Vernachlässigung in der Patientenversorgung überführen? - Berechtigte Kritik oder Missverständnis des Faches. Z Allgemeinmed $\underline{81}$, 397-404

Abholz HH, Kochen MM: Definition der Allgemeinmedizin; in: Allgemeinmedizin. Familienmedizin (Duale Reihe); hrsg. v. Kochen M; Thieme, Stuttgart 2006, 502-506

Abholz HH, Sielk M (2003): Optimierung der Schizophreniebehandlung in der Allgemeinarztpraxis (2.2.1.2.1). Endbericht 1. FP, Universität Düsseldorf. http://www.kompetenznetz-schizophrenie.de/rdkns/382.htm (zuletzt gesichtet am $11 / 06 / 2007)$

an der Heiden W, Krumm B, Müller S, Weber I, Biehl H, Schäfer M (1995): Mannheimer Langzeitstudie der Schizophrenie. Nervenarzt $\underline{66}(11), 820-827$

Arthur V, Clifford C (2004): Rheumatology: the expectations and preferences of patients for their follow-up monitoring care: a qualitative study to determine the dimensions of patient satisfaction. J Clin Nurs $\underline{13}(2), 234-242$

Aschka C, Himmel W, Ittner E, Kochen MM (2001): Sexual problems of male patients in family practice. J Fam Pract $\underline{50}(9), 773-778$

Aston J, Gschwandtner U, Riecher-Rössler A (2002): Screening zur Früherfassung von schizophrenen Psychosen in der Hausarztpraxis. Schweiz Med Forum 411, 971-974

Bad Driburger Touristik GmbH: http://www.bad-driburg.com (zuletzt gesichtet am 06/06/2007)

Barker DA, Orrell MW (1999): The Psychiatric Care Satisfaction Questionnaire: a reliability and validity study. Soc Psychiatry Psychiatr Epidemiol 34(2), 111-116

Barker DA, Shergill SS, Higginson I, Orrell MW (1996): Patients' views towards care received from psychiatrists. Br J Psychiatry 168(5), 641-646

Bengtsson-Tops A, Hansson L (1999): Clinical and social needs of schizophrenic outpatients living in the community: the relationship between needs and subjective quality of life. Soc Psychiatry Psychiatr Epidemiol 34(10), 513-518

Bindman J, Johnson S, Wright S, Szmukler G, Bebbington P, Kuipers E, Thronicroft G (1997): Integration between primary and secondary services in the care of the severely mentally ill: patients' and general practitioners' views. Br J Psychiatry $\underline{171}, 169-$ 174 
Bitzer E, Dierks ML: Wie kann man Erwartungen und Zufriedenheit der Patienten im Qualitätsmanagement berücksichtigen? Erhebungsverfahren und Erfahrungen aus der ambulanten Versorgung; in: Qualitätsmanagement in der Arztpraxis. Ergebnisse des vom Bundesministerium für Gesundheit geförderten Verbundprojektes; hrsg. v. Häussler B; Nomos, Baden-Baden 1999, 125-184

Bitzer EM, Dierks ML, Dörning H, Schwartz FW (1999): Zufriedenheit in der Arztpraxis aus Patientenperspektive. Psychometrische Prüfung eines standardisierten Erhebungsinstruments. Z Gesundheitswiss $\underline{7}(3), 196-209$

Björkman T, Hansson L, Svensson B, Berglund I (1995): What is important in psychiatric outpatient care? Quality of care from the patients ` perspective. Int J Qual Health Care $\underline{7}(4), 355-362$

Bleuler E: Handbuch der Psychiatrie - Dementia praecox oder Gruppe der Schizophrenien, Franz Deuticke, Leipzig 1911

Bodelschwingh-Hof-Mechterstädt: http://www.bodelschwingh-hof.de/ (zuletzt gesichtet am 06/06/2007)

Bogensberger S, Boss N, Jäckle R, Nawrocki P, Parzhuber S, Striebeck C, Wangerin G: Roche Lexikon Medizin, 3. Auflage, Urban \& Schwarzberg, München 1993

Bortz J, Döring N: Forschungsmethoden und Evaluation für Human- und Sozialwissenschaftler, 3. Auflage; Springer, Berlin 2002

Bosch G, Pietzcker A: Nachbehandlung krankenhausentlassener schizophrener Patienten Ergebnisse einer empirischen Untersuchung; in: Anhang zum Bericht über die Lage der Psychiatrie in der Bundesrepublik Deutschland - Zur psychiatrischen und psychotherapeutischen/psychosomatischen Versorgung der Bevölkerung. Deutsche Bundestag; hrsg. v. Enquete Kommission, 7. Wahlperiode, Drucksache 7/4200, Bonn 1975

Bottlender R, Möller HJ (2004): Schizophrene Patienten in der Allgemeinarztpraxis. Checkliste hilft bei der Früherkennung. MMW Fortschr Med $\underline{146}$ Suppl. 2, 478-482

Bowling A, Redfern J (2000): The process of outpatient referral and care: the experiences and views of patients, their general practitioners, and specialists. $\mathrm{Br} \mathrm{J}$ Gen Pract $\underline{50}$ (451), 116-120

Brugha TS, Wing JK, Smith BL (1989): Physical health of the long-term mentally ill in the community. Is there unmet need? Br J Psychiatry $\underline{155}, 777-781$

Bushnell J, McLeod D, Dowell A, Salmond C, Ramage S, Collings S, Ellis P, Kljakovic M, McBain L; MaGPle (Mental Health and General Practice Investigation) Research Group (2005): Do patients want to disclose psychological problems to GPs? Fam Pract 22(6), 631-637 
Campbell TA, Auerbach SM, Kiesler DJ (2007): Relationship of interpersonal behaviours and health-related control appraisals to patient satisfaction and compliance in a university health center. J Am Coll Health $\underline{55}(6), 333-340$

Carlsen B, Aakvik A (2006): Patient involvement in clinical decision making: the effect of GP attitude on patient satisfaction. Health Expect $\underline{9}(2), 148-157$

Carr VJ (1997): The role of the general practitioners in the treatment of schizophrenia: general principles. Med J Aust 166(2), 91-94

Carr VJ, Lewin TJ, Barnard RE, Walton JM, Allen JL, Constable PM, Chapman JL (2004): Attitudes and roles of general practitioners in the treatment of schizophrenia compared with community mental health staff and patients. Soc Psychiatry Psychiatr Epidemiol $\underline{39}(1), 78-84$

Concato J, Feinstein AR (1997): Asking patients what they like: overlooked attributes of patient satisfaction with primary care. Am J Med 102(4), 399-406

Cooper LA, Brown C, VU HT, Palenchar DR, Gonzales JJ, Ford DE, Powe NR (2000): Primary care patients' opinions regarding the importance of various aspects of care for depression. Gen Hosp Psychiatry 22(3), 163-173

Coulter A, Jenkinson C (2005): European patients' views on the responsiveness of health systems and healthcare providers. Eur J Public Health $\underline{15}$ (4), 355-360

Dagdeviren N, Akturk Z (2004): An evaluation of patient satisfaction in Turkey with the EUROPEP instrument. Yonsei Med J 45 (1), 23-28

Dathe R (1993): Patienten beim Arzt in eigener Niederlassung- ausgewählte Ergebnisse einer Befragungsstudie in allgemeinmedizinischen Praxen. Gesundheitswesen $\underline{55}(11)$, 561-566

Deutsche Gesellschaft für Psychiatrie, Psychotherapie und Nervenheilkunde (DGPPN). S3 Praxisleitlinie in Psychiatrie und Psychotherapie. Band 1- Behandlungsleitlinie Schizophrenie. Steinkopff-Verlag, Darmstadt, 2005

Diakonische Behindertenhilfe Bad Salzungen - Schmalkalden e.V.: http://www.diakonischebehindertenhilfe.de/ (zuletzt gesichtet am 06/06/2007)

Dilling $\mathrm{H}$, Weyerer S, Enders I: Patienten mit psychischen Störungen in der Allgemeinpraxis und ihre psychiatrische Überweisungsbedürftigkeit; in: Psychiatrische Epidemiologie; hrsg. v. Häfner H; Springer, Berlin 1978, 135-161

Eich PE (1990): Der praktische Arzt in sozialpsychiatrischen Konzepten der Schizophrenienbehandlung. Ther Umsch $\underline{47}(3), 225-232$ 
Escarce JJ, Kapur K, Solomon MD, Mangione CM, Lee PP, Adams JL, Wickstrom SL, Quiter E (2003): Practice characteristics and HMO enrolee satisfaction with specialty care: an analysis of patients with glaucoma and diabetic retinopathy. Health Serv Res 38(4), 1135-1155

Essex B, Doig R, Renshaw J (1990): Pilot study of records of shared care for people with mental illnesses. BMJ $\underline{300}(6737), 1442-1446$

Falkai P; Maier W (2006): Fortschritte in der neurobiologischen Erforschung in der Schizophrenie. Perspektiven für neue Therapieansätze. Nervenarzt $\underline{77}$ Suppl 3, S65-S76

Falloon IR, Ng B, Bensemann C, Kydd RR (1996): The role of general practitioners in mental health care: a survey of needs and problems. N Z Med J $\underline{109}$ (1015), 34-36

Freeman H, Alpert M (1986): Prevalence of schizophrenia in an urban population. Br J Psychiatry $\underline{149}, 603-611$

Freeman H, Cheadle AJ, Korer JR (1979): Use of hospital services by chronic schizophrenics in the community. Br J Psychiatry 134, 417-421

Fuchs J, Steinert T (2002): Inanspruchnahme professioneller Hilfe, Einweisungswege und Dauer der unbehandelten Psychose bei erstmals stationär aufgenommenen Patienten. Fortschr Neurol Psychiatr 70(1), 40-45

Fuchs J, Steinert T (2004): Dauer der unbehandelten Psychose (DUP): Ein brauchbarer Prädiktor für den Krankheitsverlauf? Fortschr Neurol Psychiatr 72(2), 79-87

Gaebel W (2001): Strategien der pharmakologischen Langzeitbehandlung schizophrener Störungen. Fortschr Neurol Psychiatr $\underline{69}$ Suppl. 2, S113-S119

Gaebel W, Falkai P: Schizophrenie, Schizotype und wahnhafte Störungen; in: Psychiatrie und Psychotherapie. Zweite, vollständig neu bearbeitete Auflage; hrsg. v. Gastpar MT, Kasper S, Linden M; Springer, Wien 2003, 97-117

Gavin B, Cullen W, O`Donoghue, Ascencio-Lane JC, Bury G, O’Callaghan E (2005): Schizophrenia in general practice: a national survey of general practitioners in Ireland. Ir J Med Sci 174(3), 38-42

Geers M: Was braucht der psychisch Kranke nach der Entlassung? Eine Befragung von Patienten psychiatrischer Einrichtungen und Vergleich mit den Empfehlungen der behandelten Ärzte. Med. Diss. Göttingen 1982

Geers M, Müller P (1984): Wünsche und Erwartungen psychiatrischer Patienten zur ambulanten Nachbehandlung- und die Empfehlung ihrer Ärzte. Psychiatr Prax 11(3), 86-91

Geiselmann B, Linden M (1989): Überweisung psychisch kranker Patienten vom Allgemeinarzt zum Nervenarzt. MMW Munch Med Wochenschr 131, 50-52 
Gericke CA, Schiffhorst G, Busse R, Häussler B (2004): Ein valides Instrument zur Messung der Patientenzufriedenheit in ambulanter haus- und fachärztlicher Behandlung: das Qualiskope-A. Gesundheitswesen $\underline{66}(11), 723-731$

Gigantesco A, Morosini P, Bazzoni A (2003): Quality of psychiatric care: validation of an instrument for measuring inpatient opinion. Int J Qual Health Care 15(1), 73-78

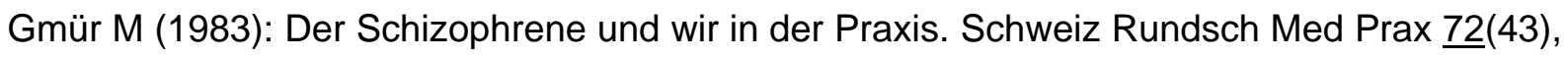
$1355-1358$

Groenewegen PP, Kerssens JJ, Sixma HJ, van der Eijk I, Boerma WG (2005): What is important in evaluating health care quality? An international comparison of user views. BMC Health Serv Res $\underline{5}(1), 16$

Grogan S, Conner M, Norman P, Willits D, Porter I (2000): Validation of a questionnaire measuring patient satisfaction with general practitioner services. Qual Health Care $\underline{9}(4), 210-215$

Grol R, Wensing M, Mainz J, Ferreira P, Hearnshaw H, Hjortdahl P, Olesen F, Ribacke M, Spenser T, Szecsenyi J (1999): Patients' priorities with respect to general practice care: an international comparison. European Task Force on Patient Evaluations of General Practice (EUROPEP). Fam Pract 16(1), 4-11

Grol R, Wensing M, Mainz J, Jung HP, Ferreira P, Hearnshaw H, Hjortdahl P, Olesen F, Reis S, Ribacke M, Szecsenyi J (2000); European Task Force on Patient Evaluations of General practice Care (EUROPEP): Patients in Europe evaluate general practice care: an international comparison. Br J Gen Pract $\underline{50}(460)$, 882-887

Gruyters T, Priebe S (1992): Die Bewertung psychiatrischer Behandlungen durch die Patienten - eine Studie zu ihrer Erfassungsmethodik und zeitlicher Stabilität. Fortschr Neurol Psychiatr $\underline{60}(4), 140-145$

Haasen C, Fritsche V (1992): Die Betreuung schizophrener und depressiver Patienten durch Hausärzte. Hamb Ärztebl $\underline{9}, 312-315$

Haasen C, Stark FM (1997): Subjektive Kriterien Schizophrener bei der Arztwahl in der ambulanten Betreuung. Psychiatr Prax 24(2), 74-76

Haasen C, Bergländer T, Stark FM (1993): Epidemiologische Aspekte bei der Betreuung Schizophrener durch Allgemeinärzte. Psycho $\underline{19}, 12-21$

Häfner H: Das Rätsel Schizophrenie. Eine Krankheit wird entschlüsselt, 2. Auflage; C.H. Beck, München 2001 
Hamann J, Cohen R, Leucht S, Busch R, Kissling W (2005): Do patients with schizophrenia wish to be involved in decisions about their medical treatment? Am J Psychiatry 162(12), 2382-2384

Hansson L (1989): The quality of outpatient psychiatric care. A survey of patient satisfaction in a sectorised care organisation. Scand J Caring Sci $\underline{3}(2), 71-82$

Hansson L, Björkman T, Berglund I (1993): What is important in psychiatric inpatient care? Quality of care from the patient's perspective. Qual Assur Health Care $\underline{5}(1), 41-47$

Hansson L, Björkman T, Svensson B (1995): The assessment of needs in psychiatric patients. Interrater reliability of the Swedish version of the Camberwell Assessment of Needs instrument and results from a cross-sectional study. Acta Psychiatr Scand $\underline{92}(4), 285-293$

Hansson L, Middelboe T, Sorgaard KW, Bengtsson- Tops A, Bjarnason O, Merinder L, Nilsson L, Sandlund M, Korkeila J, Vinding HR (2002): Living situation, subjective quality of life and social network among individuals with schizophrenia living in community settings. Acta Psychiatr Scand 106(5), 343-350

Harvey CA (1996): The Camden schizophrenia surveys. I. The psychiatric, behavioural and social characteristics of the severely mentally ill in an inner London health district. Br J Psychiatry 168(4), 410-417

Heinz A (2000): Neue Befunde für eine alte Theorie. Nervenarzt $\underline{71(1), 54-57}$

Hekkink CF, Sixma HJ, Wigersma L, Yzermans CJ, Van Der Meer JT, Bindels PJ, Brinkman K, Danner SA (2003): QUOTE-HIV: an instrument for assessing quality of HIV care from the patients' perspective. Qual Saf Health Care 12(3), 188-193

Himmel W, Kochen MM, Kapmeyer A, Rogausch A (2003): Optimierung der Schizophreniebehandlung in der Allgemeinarztpraxis (2.2.1.2.1.). Endbericht 1. FP, Universität Göttingen: http://www.kompetenznetz-schizophrenie.de/rdkns/382.htm (zuletzt gesichtet am 11/06/2006)

Himmel W, Ewert W, Klein R: Der Patient im Kontext der Familie; in: Allgemeinmedizin. Familienmedizin (Duale Reihe); hrsg. v. Kochen M; Thieme, Stuttgart 2006, 524-536

Hoffmann K, Priebe S (1996): Welche Bedürfnisse nach Hilfe haben Langzeitpatienten? Probleme der Selbst- und Fremdbeurteilung von "Needs“. Fortschr Neurol Psychiatr $\underline{64}(12), 473-481$

Hübner-Liebermann B, SpießI H, Cording C (2005): Wer kommt vorher, wer geht wohin? Behandlungswege stationärer- psychiatrischer Patienten. Nervenarzt $\underline{76}$ (7), 856-864 
ICD-10-WHO (2006): http://www.dimdi.de/static/de/klassi/diagnosen/icd10/htmlamtl2006/fricd.htm (zuletzt gesichtet am 18/07/2006)

Infante FA, Proudfoot JG, Powell Davies G, Bubner TK, Holton CH, Beilby JJ, Harris MF (2004): How people with chronic illnesses view their care in general practice: a quailtative study. Med J Aust 181(2), 70-73

Jablenski A, McGrath J, Herrman H, Castle D, Gureje O, Evans M, Carr V, Morgan V, Korten A, Harvey C (2000): Psychotic disorders in urban areas: an overview of the Study on Low Prevalence Disorders. Aust N Z J Psychiatry 34(2), 221-236

Jacobi CE, Boshuizen HC, Rupp I, Dinant HJ, Geertrudis A, van den Bos GA (2004): Quality of rheumatoid arthritis care: the patient's perspective. Int J Qual Health Care $\underline{16}(1)$, 73-81

Jäger M, Riedel M, Möller HJ (2006): Früherkennung, Rezidivprophylaxe, Therapie körperlicher Beschwerden. Die Rolle des Hausarztes im Management der Schizophrenie. MMW Fortschr Med 148 Suppl. 2, 39-41

Johnstone EC, Owens DG, Gold A, Crow TJ, Macmillian JF (1984): Schizophrenic patients discharged from hospital-a follow-up study. Br J Psychiatry $\underline{145}, 586-590$

Jung HP, Wensing M, Olesen F, Grol R (2002): Comparison of patients' and general practitioners' evaluations of general practice care. Qual Saf Health Care 11(4), 315-319

Kallert TW, Leisse M (2000): Betreuungsbedürfnisse schizophrener Patienten im Jahr nach Klinikentlassung in der Versorgungsregion Dresden. Fortschr Neurol Psychiatr $\underline{68}(4)$, 176-187

Kendrick T, Sibbald B, Burns T, Freeling P (1991): Role of general practitioners in care of long term mentally ill patients. BMJ $\underline{302}(6775), 508-510$

Kendrick T, Burns T, Freeling P, Sibbald B (1994): Provision of care to general practice patients with disabling long-term mental illness: a survey in 16 practices. Br J Gen Pract 44(384), 301-305

Kendrick T, Burns T, Garland C, Greenwood N, Smith P (2000): Are specialist mental health services being targeted on the most needy patients? The effects of setting up special services in general practice. Br J Gen Pract $\underline{50}$ (451), 121-126

Kerssens JJ, Groenewegen PP, Sixma HJ, Boerma WG, van der Eijk I (2004): Comparison of patient evaluations of health care quality in relation to WHO measures of achievement in 12 European countries. Bull World Health Organ 82(2), 106-114

King M (1992): Management of patients with schizophrenia in general practice. $\mathrm{Br} \mathrm{J}$ Gen Pract $\underline{42}(361), 310-311$ 
King M, Nazareth I (1996): Community care of patients with schizophrenia: the role of the primary health care team. Br J Gen Pract $\underline{46}(405), 231-237$

Kjeken I, Dagfinrud H, Mowinckel P, Uhlig T, Kvien TK, Finset A (2006): Rheumatology care: Involvement in medical decisions, received information, satisfaction with care, and unmet health care needs in patients with rheumatoid arthritis and ankylosing spondylitis. Arthritis Rheum $\underline{55}(3), 394-401$

Klingenberg A, Bahrs O, Szecsenyi J (1996): Was wünschen Patienten vom Hausarzt? Z Allgemeinmed $\underline{72}, 180-186$

Klingenberg A, Bahrs O, Szecsenyi J (1999): Wie beurteilen Patienten Hausärzte und ihre Praxen? Z Ärztl Fortbild Qualitätssich $\underline{93}(6), 473-445$

Köhn D, Niedersteberg A, Wieneke A, Bechdolf A, Pukrop R, Ruhrmann S, Schultze-Lutter F, Maier W, Klosterkötter J (2004 a): Frühverlauf schizophrener Ersterkrankungen mit langer Dauer der unbehandelten Erkrankung - eine vergleichende Studie. Fortschr Neurol Psychiatr $\underline{72}(2), 88-92$

Köhn D, Pukrop R, Niedersteberg A, Schultze-Lutter F, Ruhrmann S, Bechdolf A, Berning J, Maier W, Klosterkötter J (2004 b): Wege in der Erstbehandlung: Hilfesuchverhalten schizophrener Ersterkrankter. Fortschr Neurol Psychiatr 72(11), 635-642

Krankenhaus Bad Salzungen gGmbh: http://www.kh-badsalzungen.de/ (zuletzt gesichtet am 06/06/2007)

Kravitz RL, Cope DW, Bhrany V, Leake B (1994): Internal medicine patients' expectations for care during office visits. J Gen Intern Med $\underline{9}(2), 75-81$

Laine C, Davidoff F, Lewis CE, Nelson EC, Nelson E, Kessler RC, Delbanco TL (1996): Important elements of outpatient care: a comparison of patients' and physicians' opinions. Ann Intern Med 125(8), 640-645

Lamnek S: Qualitative Sozialforschung. 4.Auflage; Beltz Verlag, Weinheim 2005

Lang FH, Johnstone EC, Murray GD (1997): Service provision for people with schizophrenia. II. Role of the general practitioner. Br J Psychiatry $\underline{171}, 165-168$

Längle G, Mayenberger M (2000): Die Rolle der Klinik im Verlauf schizophrener Erkrankungen. Gesundheitwesen 62(1), 9-14

Lester H, Tritter JQ, England E (2003): Satisfaction with primary care: the perspectives of people with schizophrenia. Fam Pract 20(5), 508-513

Lester H, Tritter JQ, Sorohan H (2005): Patients' and health professionals' views on primary care for people with serious mental illness: focus group study. BMJ $\underline{330}(7500), 1122-$ 1126 
Lewin TJ, Carr VJ (1998): Rates of treatment of schizophrenia by general practitioners. A pilot study. Med J Aust 168(4), 166-169

Linden M, Maier W, Achberger M, Herr R, Helmchen H, Benkert O (1996): Psychische Erkrankungen und ihre Behandlung in Allgemeinarztpraxen. Nervenarzt 67(3), 205-215

Little P, Everitt H, Williamson I, Warner G, Moore M, Gould C, Ferrier K, Payne S (2001): Preferences of patients for patient centred approach to consultation in primary care: observational study. BMJ $\underline{322}$ (7284), 468-472

Maurer K, Hörrmann F, Häfner H (2006): Schizophrenie - Erste Anzeichen rechtzeitig erkennen. Allgemeinarzt $\underline{19}, 14-16$

McGrath J, Saha S, Welham J, El Saadi O, MacCauley C, Chant D (2004): A systematic review of the incidence of schizophrenia: the distribution of rates and the influence of sex, urbanicity, migrant status and methodology. BMC Med 2(13)

Meller I, Fichter MM, Rehm Jürgen: Krankheitsverhalten: Inanspruchnahme medizinischer Dienste; in: Verlauf psychischer Erkrankungen in der Bevölkerung; hrsg. v. Fichter MM; Springer, Berlin 1990, 232-255

Messer T, Tiltscher C, Schmauss M (2006): Polypharmazie in der Behandlung der Schizophrenie. Fortschr Neurol Psychiatr $\underline{74}(7), 377-391$

Meyer T, Franz M (2006): Vorstellungen zur Lebensqualität schizophrener Erkrankter. Der Zusammenhang mit ihren quantitativen Lebensqualitätsurteilen. Psychiatr Prax $\underline{33}$ (7), 344-349

Möller HJ, Laux G, Deister A: Psychiatrie und Psychotherapie, 3. überarbeitete Auflage, Thieme, Stuttgart 2005

Möller-Leimkühler AM, Dunkel R (2003): Zufriedenheit psychiatrischer Patienten mit ihrem stationären Aufenthalt- Ergebnisse einer Stichtagserhebung. Nervenarzt 74(1), 40-47

Müller P, Gaebel W, Bandelow B, Köpcke W, Linden M, Müller- Spahn F, Pietzcker A, Tegeler J (1998): Zur sozialen Situation schizophrener Patienten. Nervenarzt $\underline{69}(3), 204-$ 209

Nazareth I, King M, Davies S (1995): Care of schizophrenia in general practice: the general practitioner and the patient. Br J Gen Pract 45(396), 343-347

Pajonk FG, Wobrock T, Falkai P (2005): Langzeittherapie der Schizophrenie. Teil 1: Hintergrund, Behandlungsziele, Pharmakotherapie. Fortschr Neurol Psychiatr 73(3), 161174 
Platz C, Umbricht DS, Cattapan-Ludewig K, Dvorsky D, Arbach D, Brenner HD, Simon AE (2006): Help-seeking pathways in early psychosis. Soc Psychiatry Psychiatr Epidemiol $\underline{41}(12), 967-974$

Präsentation der Hörselbergwerkstatt: persönlich überreicht von Jörn Köhler am 07/06/2007

Präsentation der Integ gGmbH(2003), Bad Driburg; Kongress „Wege zur Integration behinderter Menschen ins Arbeitsleben“: persönlich überreicht bekommen am 04/03/2004

Präsentation der Integ gGmbH(2007), Integ. „Eine Idee setzt sich durch. Im Mittelpunkt steht der Mensch“: persönlich überreicht bekommen am 31/05/2007

Priebe S, Gruyters T, Heinze M, Hoffmann C, Jäkel A (1995): Subjektive Evaluationskriterien in der psychiatrischen Versorgung- Erhebungsmethode für Forschung und Praxis. Psychiatr Prax 22(4), 140-144

Prince M, Phelan M (1994): A postal survey of the incidence and management of new cases of schizophrenia in primary care. J Ment Health $\underline{3}$, 91-98

Redsell S, Stokes T, Jackson C, Hastings A, Baker Richard (2007): Patients' accounts of the differences in nurses' and general practitioners' roles in primary care. J Adv Nurs $\underline{57}(2), 172-80$.

Rössler-Riecher A (1997): 50 Jahre nach Manfred Bleuler - Was wissen wir heute über die Spätschizophrenie(n)? Nervenarzt $\underline{68}(3), 159-170$

Ruggeri M, Lasalvia A, Bisoffi G, Thornicroft G, Vazquez-Barquero JL, Becker T, Knapp M, Knudsen HC, Schene A, Tansella M and the Epsilon Study Group (2003): Satisfaction with mental health services among people with schizophrenia in five European sites: results from the EPSILON Study. Schizophr Bull 29, 229-245

Ruhrmann S, Schultze-Lutter F, Klosterkötter J (2003): Schizophrenie früh erkennen. Der Hausarzt stellt die Weichen! Allgemeinarzt 17, 1294-1301

Saha S, Chant D, Welham J, McGrath J (2005): A systematic review of the prevalence of schizophrenia. PLoS Med 2(5), 0413-0433

Salomon P, Quine J (1989): Patients 'Interventions in Primary Care: Measurement and Preliminary Investigation. Psychol Health $\underline{3}, 103-110$

Schattner A, Rudin D, Jellin N (2004): Good physicians from the perspective of their patients. BMC Health Serv Res $\underline{4}(1), 26$

Schattner A, Bronstein A, Jellin N (2006): Information and shared decision-making are top patients' priorities. BMC Health Serv Res $\underline{6}, 21-26$ 
Schildmann J, Bauer A, Tilmann A, Vollmann J (2003): Aufklärung und Einwilligung zur Psychopharmakotherapie aus Sicht schizophrener und depressiver Patienten. Fortschr Neurol Psychiatr 71(5), 265-270

Schmid R, Neuner T, Cording C, SpießI H (2006): Lebensqualität schizophrener Erkrankter und ihr Zusammenhang mit Krankheitsbewältigungsstrategien und Behandlungsaspekten. Psychiatr Prax 33(7), 337-343

Scholl A: Die Befragung: Sozialwissenschaftliche Methode und kommunikationswissenschaftliche Anwendung, UVK Verlagsgesellschaft, Konstanz 2003

Schultz SK, Andreasen NC (1999): Schizophrenia. Lancet $\underline{353}$ (9162), 1425-1430

Sielk M, Abholz HH (2005): Warum bezeichnen Allgemeinärzte andere Patienten als depressiv als Psychiater es tun? Z Allgemeinmed $\underline{81}$, 486-490

Sielk M, Janssen B (2004): Hausärztlich relevante Aspekte bei der Diagnostik und Therapie der Schizophrenie. Z Allgemeinmed 80, 470-482

Simon AE, Lauber C, Ludewig K, Braun-Scharm H, Umbricht DS (2005): General practitioners and schizophrenia: results from a Swiss survey. Br J Psychiatry 187, 275-281

Sixma HJ, van Campen C, Kerssens JJ, Peters L (2000): Quality of care from the perspective of elderly people: the QUOTE-elderly instrument. Age Ageing 29(2), 173-178

Spieß। H, Cording C (2000): Zusammenarbeit niedergelassener Allgemeinärzte und Nervenärzte mit der psychiatrischen Klinik- Eine Literaturübersicht. Fortschr Neurol Psychiatr $\underline{68}(5), 206-215$

SpießI H, Leber C, Kaatz S, Cording C (2002): Was erwarten Patienten von einer psychiatrischen Abteilung an einem Allgemeinkrankenhaus? Psychiatr Prax 29(8), 417-419

Stadtportrait Eisenach: http://www.eisenach.de (zuletzt gesichtet am 06/06/2007)

Stark FM, Stolle R (1994): Schizophrenie: Subjektive Krankheitstheorien - Eine explorative Studie. Psychiatr Prax 21(2), 74-78

Steine S, Finset A, Laerum E (2001): A new, brief questionnaire (PEQ) developed in primary health care for measuring patients' experience of interaction, emotion and consultation outcome. Fam Pract 18

St. Georg Klinikum Eisenach gGmbh: http://www.stgeorgklinikum.de (zuletzt gesichtet am 06/06/2007)

St. Josef-Hospital Bad Driburg: http://www.kh-driburg.de/ (zuletzt gesichtet am 06/06/2007) 
Thapinta D, Anders RL, Wiwatkunupakan S, Kitsumban V, Vadtanapong S (2004): Assessment of patient satisfaction of mentally ill patients hospitalized in Thailand. Nurs Health Sci $\underline{6}(4), 271-277$

Valori R, Woloshynowch M, Bellenger N, Aluvihare V, Salmon P (1996): The Patient Requests Form: a way of measuring what patients want from their general practitioner. $\mathrm{J}$ Psychosom Res $\underline{40}(1)$, 87-94

Van Campen C, Sixma HJ, Kerssens JJ, Peters L (1997): Assessing noninstitutionalized asthma and COPD patients' priorities and perceptions of quality of health care: the development of the QUOTE-CNSLD instrument. J Asthma, $\underline{34(6), 531-538}$

Van Campen C, Sixma HJ, Kerssens JJ, Peters L, Rasker JJ (1998): Assessing patients' priorities and perceptions of the quality of health care: the development of the QUOTERheumatic-Patients instrument. Br J Rheumatol 37(4), 362-368

Vauth R, Loh A, Sitta A, Schweiger C, Niebling W (2004): Früh- und Basisintervention bei Patienten mit schizophrenen Störungen. Ein interaktives und leitlinienorientiertes Trainingsprogramm für die Hausarztpraxis. Nervenarzt $\underline{75}(9), 873-881$

Vedsted P, Mainz J, Lauritzen T, Olesen F (2002): Patient and GP agreement on aspects of general practice care. Fam Pract 19(4), 339-343

Verdoux H, Cougnard A, Grolleau S, Besson R, Delcroix F (2005): How do general practitioners manage subjects with early schizophrenia and collaborate with mental health professionals? A postal survey in South-Western France. Soc Psychiatry Psychiatr Epidemiol 40(11), 892-898

Verhaak PF, van den Brink-Muinen A, Bensing JM, Gask L (2004): Demand and supply for psychological help in general practice in different European countries: access to primary mental health care in six European countries. Eur J Public Health 14(2), 134140

Von Korff M, Tiemens B (2000): Individualized stepped care of chronic illness. West J Med 172(2), 133-7

Von Korff M, Nestadt G, Romanoski A, Anthony J, Eaton W, Merchant A, Chahal R, Kramer M, Folstein M, Gruenberg E (1985): Prevalence of treated and untreated DSM-III schizophrenia. Results of a two-stage community survey. J Nerv Ment Dis $\underline{173}(10)$, 577-581

Watts CA (1973): Review of schizophrenics in a rural practice over 26 years. BMJ 1973,1, 465-469 
Wensing M, Jung HP, Mainz J, Olesen F, Grol R (1998): A systematic review of the literature on patient priorities for general practice care. Part 1: Description of the research domain. Soc Sci Med $\underline{47}(10), 1573-1588$

Weyerer S, Dilling H (1983): Psychisch Kranke in Allgemeinpraxen. Eine psychiatrischepidemiologische Untersuchung im Landkreis Traunstein. Fortschr Med 101(15), 670675

Wiersma D, Nienhuis FJ, Giel R, Slooff (1998): Stability and change in needs of patients with schizophrenic disorders: a 15- and 17-year follow-up from first onset of psychosis, and a comparison between 'objective' and 'subjective' assessments of needs for care. Soc Psychiatry Psychiatr Epidemiol 33(2), 49-56

Williams S, Calnan M (1991): Key determinants of consumer satisfaction with general practice. Fam Pract $\underline{8}(3), 237-242$

Williams S, Weinman J, Dale J, Newman S (1995): Patient expectations: what do primary care patients want from the GP and how far does meeting expectations affect patient satisfaction? Fam Pract 12(2), 193-201

Wobrock T, Pajonk FG, Falkai P (2004): Schizophrenie: Teil I Epidemiologie, Ätiopathogenese, Symptomatologie. Fortschr Neurol Psychiatr $\underline{72(2), 98-113}$

Wolf MH, Putnam SM, James SA, Stiles WB (1978): The Medical Interview Satisfaction Scale: development of a scale to measure patient perceptions of physician behavior. J Behav Med 11(4), 391-401

Yates WR (1986): The National Institute of Mental Health epidemiologic study: implications for family practice. J Fam Pract 22(3), 251-255

Zintl-Wiegand A, Schmidt-Maushart C, Leisner R, Cooper B: Psychische Erkrankungen in Mannheimer Allgemeinpraxen. Eine klinische und epidemiologische Untersuchung; in: Psychiatrische Epidemiologie; hrsg. v. Häfner H; Springer, Berlin 1978, 111-135 


\section{Anhang}

\subsection{Arztanschreiben}

Abteilung Allgemeinmedizin

Direktor: Univ.-Prof. Dr. med. Michael M. Kochen, MPH, FRCGP Abteilung Allgemeinmedizin

Anschrift des Arztes

Anschrift des Arztes,
Abt. Allgemeinmedizin

Priv.-Doz. Dr.

Wolfgang Himmel

Humboldtallee 38

37073 Göttingen

Telefon: (0551) 39-2648

Telefax: (0551) 39-9530

e-mail:

whimmel@gwdg.de

wir bitten Sie um Unterstützung bei unserer Studie über die „Erwartungen von schizophrenen Patienten an hausärztliche und psychiatrische Behandlung“.

Bei dieser Studie wird unsere Doktorandin Kirstin Kühmel Patienten befragen. Frau Kirstin Kühmel wird sich mit Ihnen in den kommenden Tagen telefonisch in Verbindung setzen und Ihnen die Studie näher vorstellen.

Unsere Bitte an Sie: Informieren Sie in Frage kommende Patienten über die Möglichkeit zur Teilnahme an der Studie. Wie dies im Einzelnen ablaufen soll, wird Ihnen Frau Kühmel erläutern. Die Vermittlung eines Interviews würdigen Patienten erfahrungsgemäß als ein Zeichen besonderen Engagements der Praxis. Das EthikKomitee der Georg-August-Universität Göttingen hat die Studie genehmigt. Selbstverständlich melden wir Ihnen alle Ergebnisse in anonymisierter Form zurück.

Außer dieser Vermittlung wird keine Arbeit auf Sie oder Ihre Arzthelferinnen zukommen. Über Ihre Unterstützung würden wir uns sehr freuen und stehen für Rückfragen und Anregungen gerne zur Verfügung (Tel. Dipl.-Psych. A. Rogausch; Mo - Mi 0551399537).

Mit bestem Dank und

freundlichen Grüßen

Priv.-Doz. Dr. W. Himmel Dipl.-Psych. Anja Rogausch cand. med. Kirstin Kühmel 


\subsection{Patientenanschreiben}

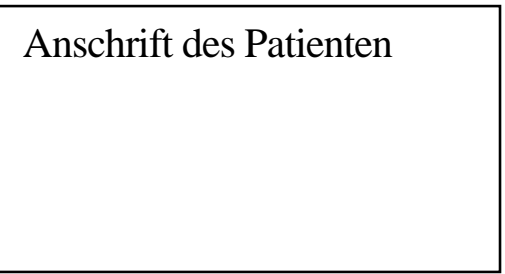

Liebe Patientin, lieber Patient,

ich möchte Sie als Ihr Hausarzt um die Teilnahme an einem Forschungsprojekt bitten, das von der Abteilung Allgemeinmedizin der Georg-August-Universität Göttingen durchgeführt wird. Dabei geht es um „Erwartungen von schizophrenen Patienten an die hausärztliche und psychiatrische Behandlung". Die Forschungsarbeit soll zur Verbesserung der Patientenzufriedenheit und -versorgung beitragen, was mir (und sicherlich auch Ihnen) am Herzen liegt.

Kirstin Kühmel, Doktorandin der Abteilung Allgemeinmedizin, wird Sie in einem sicher interessanten Gespräch befragen (Dauer: ca. 45 min) - zum Beispiel darüber, was Ihnen in der Behandlung durch Hausärzte und Psychiater wichtig ist und inwieweit Sie mit uns zufrieden sind.

Die Doktorandin unterliegt selbstverständlich der Schweigepflicht. Ich selbst erfahre nicht, ob Sie an der Studie teilnehmen bzw. wie Sie die Fragen beantworten. In der beiliegenden Einverständniserklärung finden Sie nähere Einzelheiten zu der Studie.

Wenn Sie an der Studie teilnehmen möchten, senden Sie bitte die Einverständniserklärung an die Abteilung Allgemeinmedizin (mit dem Briefumschlag „Gebühr zahlt Empfänger"). Ihre Entscheidung für oder gegen die Studienteilnahme hat keinerlei Einfluss auf die Behandlung Ihrer Erkrankung. Sie können Ihr Einverständnis jederzeit ohne Erklärung wieder zurückziehen.

Bei auftretenden Fragen wenden Sie sich bitte an mich oder an Frau Dipl.-Psych. Anja Rogausch (Abteilung Allgemeinmedizin, telefonisch erreichbar: Mo - Mi 0551399537, die Sie gerne auch zurückruft).

Mit besten Grüßen

$\mathrm{Ihr}$ 


\subsection{Informed consent}

\section{GEORG - AUGUST - UNIVERSITÄT GÖTTINGEN}

Bereich Humanmedizin - Universitätsklinikum - Medizinische Fakultät

\section{Abteilung Allgemeinmedizin}

Univ.-Prof. Dr. med. Michael M. Kochen, MPH, FRCGP

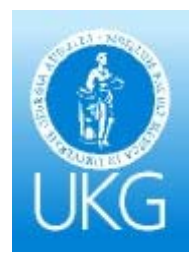

Patientenaufklärung zur Studie

“KRITERIEN DER ARZTWAHL UND -BEWERTUNG

PSYCHIATRISCHER PATIENTEN“

Sehr geehrte Patientin, sehr geehrter Patient,

die Abteilung Allgemeinmedizin führt ein wissenschaftliches Projekt durch, bei dem es um die Sicht von Patienten auf die Behandlung durch Haus- und Nervenärzte geht, über die bisher wenig bekannt ist. Ihre Wünsche an die Behandlung kennen zu lernen und Ihre Einschätzung zu erfahren, in welchen Bereichen Sie sich Unterstützung wünschen, kann dabei helfen, die Behandlung in Zukunft zu verbessern. Daher möchten wir Sie um die Teilnahme an einem persönlichen Gespräch mit einer Mitarbeiterin unserer Abteilung bitten.

\section{Was sind die Ziele des Forschungsprojektes?}

Ein wesentliches Ziel dieser Untersuchung besteht darin, die Versorgung stärker an den Bedürfnissen der Nutzer, also an Ihnen, zu orientieren. Zu diesem Zweck wollen wir Sie gerne zu einigen Bereichen befragen, von denen wir annehmen, dass sie in einem engen Zusammenhang mit psychischen Problemen stehen können. Hierzu gehören Fragen zu Ihren Problemen und Beschwerden, zu möglichen Therapiezielen sowie zu Ihrer Zufriedenheit mit der Behandlung.

\section{Was kommt bei einer Teilnahme auf Sie zu:}

- Sie senden ein Exemplar der Einverständniserklärung im beiliegenden Umschlag an die Abteilung Allgemeinmedizin der Universität Göttingen zurück. 
- Eine Doktorandin der Abteilung Allgemeinmedizin (Frau cand. med. Kirstin Kühmel) ruft Sie an, informiert Sie ausführlich über das Projekt und beantwortet mögliche weitere Fragen. Sie vereinbart mit Ihnen einen Termin (ca. 3/4 Stunde) für ein Gespräch, das je nach Absprache in der Arztpraxis, Klinik oder bei Ihnen zu Hause stattfinden kann.

- Bei diesem Termin wird die Doktorandin, Frau Kühmel, ein kurzes Gespräch mit Ihnen führen und einen Fragebogen mit Ihnen besprechen.

- Alle Daten werden streng vertraulich behandelt und nur in anonymisierter Form verwendet. Alle Mitarbeiter unterliegen vollständig der Schweigepflicht, sämtliche Bestimmungen des Datenschutzrechts werden selbstverständlich beachtet.

\section{Als Teilnehmer/in beachten Sie bitte folgendes:}

- Die Teilnahme ist freiwillig, eine Nichtteilnahme bringt keinerlei Nachteile für Ihre weitere Therapie mit sich. Sie können Ihr einmal gegebenes Einverständnis jederzeit ohne Angabe von Gründen zurückziehen und auch die Beantwortung von Fragen ablehnen, ohne dass Ihnen daraus irgendwelche Nachteile entstehen.

- Bei Fragen können Sie sich gerne an die Abteilung Allgemeinmedizin der Universität Göttingen (Tel.-Nr. siehe oben; Frau Dipl.-Psych. Anja Rogausch, cand. Med. Kirstin Kuehmel) wenden. 


\section{GEORG - AUGUST - UNIVERSITÄT GÖTTINGEN}

Bereich Humanmedizin - Universitätsklinikum - Medizinische Fakultät

\section{Abteilung Allgemeinmedizin}

Univ.-Prof. Dr. med. Michael M. Kochen, MPH, FRCGP

\section{Patienteneinverständniserklärung zur Studie}

“ KRITERIEN DER ARZTWAHL UND -BEWERTUNG

PSYCHIATRISCHER PATIENTEN“

Ich, (Name des Patienten), wurde vollständig über das Forschungsprojekt mit dem o.g. Titel aufgeklärt. Ich habe den Aufklärungstext gelesen und verstanden. Ich hatte die Möglichkeit, Fragen zu stellen, und habe die Antworten verstanden und akzeptiere sie.

Ich hatte ausreichend Zeit, mich zur Teilnahme an dieser Studie zu entscheiden und weiß, dass die Teilnahme an dieser Studie freiwillig ist. Ich weiß, dass ich jederzeit und ohne Angaben von Gründen diese Zustimmung widerrufen kann, ohne dass sich dieser Entschluss nachteilig auf die weitere Behandlung durch meinen Arzt auswirken wird. Ich bin damit einverstanden, dass die im Rahmen der Studie erhobenen Daten in anonymisierter Form gespeichert und zu Forschungszwecken verwendet werden.

Ich habe eine Kopie der Patienteninformation und dieser Einwilligungserklärung erhalten. Ich erkläre bin damit einverstanden, dass mich eine Projektmitarbeiterin zur Terminabsprache anruft und erkläre hiermit meine freiwillige Teilnahme an der Studie.

Ich bin erreichbar unter folgender Telefonnummer:

am besten erreichbar um Uhr 


\subsection{Patientendaten}

Interviewer: Datum:

Fallnummer

\section{PATIENTENDATEN}

\begin{tabular}{|ll|}
\hline $\begin{array}{l}\text { Muttersprache: } \\
\text { deutsch }\end{array}$ & Geschlecht: \\
$\begin{array}{l}\text { andere Muttersprache, gute deutsche Sprachkenntnisse } \\
\text { andere Muttersprache, schlechte deutsche Sprachkenntnisse }\end{array}$ & $\square$ \\
$\begin{array}{l}\text { sprachliche Verständigung aus anderen Grüden unmöglich, oder } \\
\text { erschwert (gehörlos, aphonisch o.ä.) }\end{array}$ & Weburtsjahr: 19_ \\
unbekannt / unklar
\end{tabular}

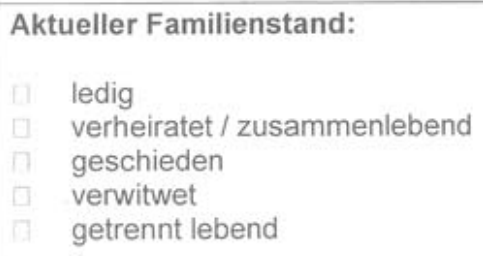

\section{feste Partnerschaft:}

$$
\text { ja }
$$

nein

Alter bei erstmaligem Auftreten der psych. Beschwerden: $\square \square$ Jahre unbekannt

Erste psychiatrische Behandlung (Jahresangabe, evtl. geschätzt): $\square \square \square \square \square$ unbekannt

Anzahl der stationären Aufenthalte: (eventuell geschătzt): $\square \square \square$ unbekannt

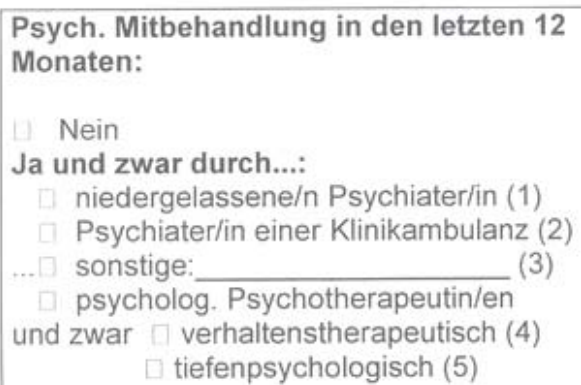

Wer ist Hauptansprechpartner für psych. Probleme (Ziffern s.o.; Hausarzt=6):

Wer ist Hauptansprechpartner in Fragen der psychopharm. Medikation, falls vorhanden (Ziffern s.o.; Hausarzt=6):

Hausarzt bekannt seit: Jahren

Psychiater / Psychologe (bitte zutreffend unterstreichen) bekannt seit: Jahren

Diagnose in eigenen Worten:
Höchster Schulabschluss:

keiner

unbekannt

Hauptschulabschluss

Mittlere Reife

Fach-/ Hochschulreife

Derzeitige berufliche Situation:

Vollzeit berufstätig seit mehr als 6 Monaten

Teilzeit berufstätig seit mehr als 6 Monaten

in beschützter Beschäftigung

Hausfrau/ -mann

in Ausbildung / Studium

arbeitslos gemeldet

Erwerbs-/ Berufsunfähigkeits-/ Früh-/ Altersrentner anderweitig ohne berufliche Beschäftigung unbekannt/unklar

Wohnsituation:

alleine / in nicht-therapeutischer WG

in eigener Familie/Partnerschaft

bei Eltern (Herkunftsfamilie)

therapeutische WG oder psychiatrische

Familienpflege

psychiatrische Übergangseinrichtung, Altersheim

Notunterkunft (z.B. Asylantenheim,

Frauenhaus, Obdachlosenheim)

unbekannt

Teilnahme an Selbsthilfegruppe? 
Datum:

Fallnummer:

\section{Größe des Wohnorts:}

$<5.000$ Einwohner

$5.000-25.000$ Einwohner

25.000 - 100.000 Einwohner

$>100.000$ Einwohner

\section{Besteht eine gesetzliche Betreuung?}

ja nein unbekannt

Bereiche (Mehrfachantworten möglich):

unbekannt Aufenthalt Behörden

Gesundheit Vermögen Wohnung

\section{Gegenwärtige Medikation:}

A) Orale Medikation:

Präparat1 (Handelsname):

Tagesdosis (in mg):

Wird eingenommen seit:

(Monat, Jahr)

Präparat 2 (Handelsname)

Tagesdosis (in mg):

Wird eingenommen seit:

(Monat, Jahr)

Präparat 3 (Handelsname):

Tagesdosis (in mg):

Wird eingenommen seit: (Monat, Jahr)

B) Depot-Medikation, wenn bekannt:

Präparat (Handelsname):

Depot-Konzentration (in \%):

Depot-Dosis (in $\mathrm{ml}$ ):

Frequenz der Depotgabe:

alle

Tage.

Wird eingenommen seit:

(Monat, Jahr)

Interview vermittelt über:

Hausarzt Nervenarzt Klinik/Werkstatt sonstiges:

Ort des Interviews:

bei Patient Praxis Hausarzt Praxis Nervenarzt Klinik:_ sonstiges:

Bemerkungen:

CGI: 
9.5. Einstiegs- und Abschlussfragen

\section{Einstiegsfragen:}

1. Überlegen Sie bitte kurz in welchen Situationen bzw. mit welchem Anliegen Sie sich gefühlsmäßig eher an den Hausarzt bzw. an den Nervenarzt wenden?

2. Wieso ist das so? Nennen Sie Gründe dafür. Motive für den Vorzug des einen Arztes gegenüber dem anderen?

Abschlussfragen:

3. Nennen Sie bitte einige Punkte, die Sie sich besonders an ihrem Hausarzt wünschen?

4. Nennen Sie einige Punkte, die Ihnen bei Ihrem Nervenarzt wichtig erscheinen? 


\subsection{Fragebogen Teil 1}

\section{Teil 1}

Ist es Ihnen beim Hausarzt oder Psychiater wichtiger, dass...

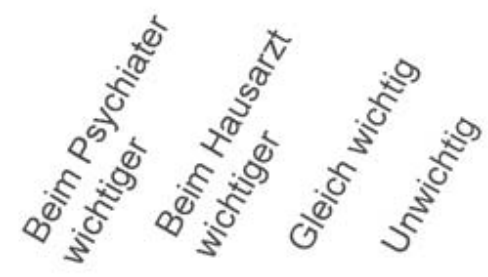

-...der Arzt in der Nähe Ihres Wohnortes praktiziert

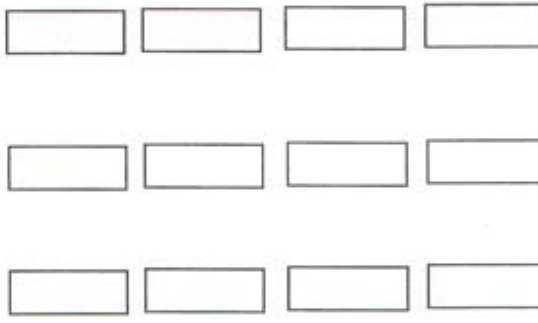

...in der Praxis eine angenehme

Atmosphäre (z.B. keine Hektik) herrscht

...Sie den Arzt lange kennen

- ...es möglich ist, schnell einen Termin zu bekommen (innerhalb von 2-3 Tagen)

... der Arzt auch außerhalb der üblichen Sprechzeiten erreichbar ist (z.B. abends, nachts, am Wochenende)

- ...der Arzt Hausbesuche macht

...der Arzt sich Zeit für Sie nimmt (z.B. auch einmal mehr als $15 \mathrm{~min}$.) ..der Arzt Ihnen das Ergebnis einer Untersuchung erklärt
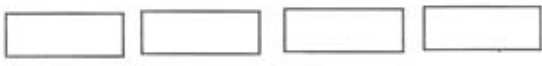

- ...der Arzt Sie umfassend über die seelische Erkrankung informiert

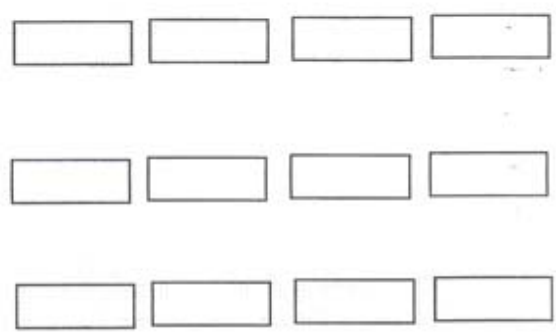

-...der Arzt es Ihnen leicht macht, über belastende Probleme zu sprechen

...der Arzt Sie in die Entscheidungen über die medizinische Behandlung mit einbezieht

- ...der Arzt Ihnen den Zweck von Untersuchungen / Behandlungen erklärt
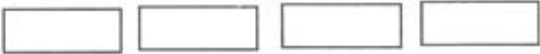
...der Arzt gut zuhören kann ..der Arzt Ihnen mit Respekt begegnet

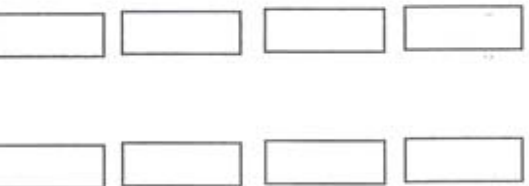


(Fortsetzung)

\section{Ist es Ihnen beim Hausarzt oder Psychiater wichtiger, dass...}

- ...der Arzt von sich aus nach seelischen Belastungen fragt

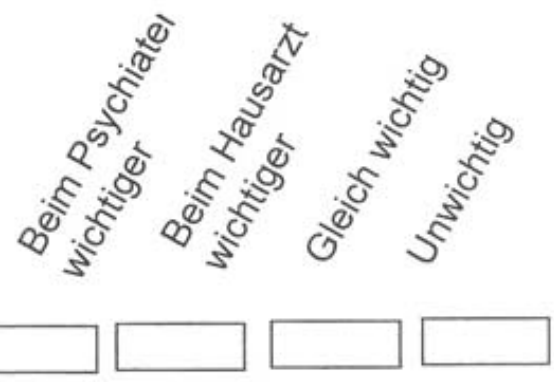

....der Arzt darauf Rücksicht nimmt, wie Sie sich die Behandlung Ihrer seelischen Erkrankung vorstellen

- ...der Arzt auf die vertrauliche Behandlung persönlicher Angaben achtet

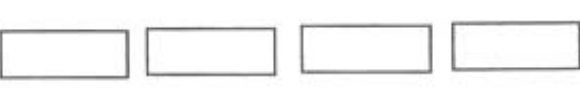

... der Arzt Sie ermuntert, Fragen zu stellen

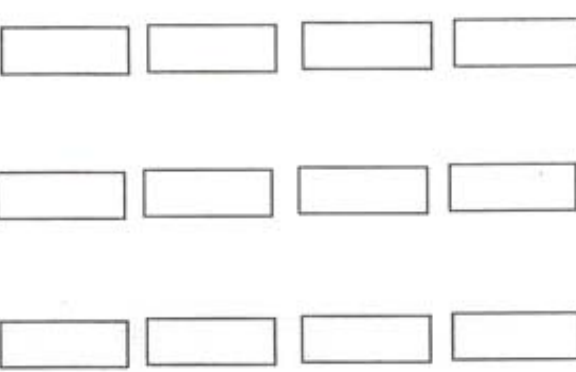

...der Arzt Ihre Angehörigen unterstützt und berät

- ...der Arzt Sie sorgfältig körperlich untersucht

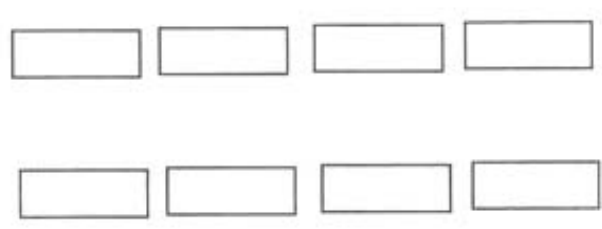
überweist

- ...die Wartezeiten in der Praxis kurz sind (nicht länger als $30 \mathrm{~min}$.)

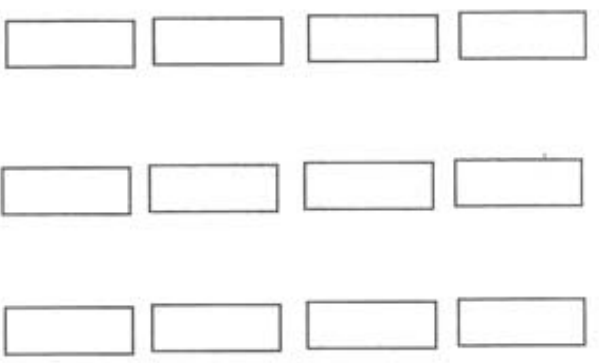

...der Arzt darauf eingeht, wenn Sie die von ihm verschriebenen Medikamente nicht eingenommen haben

- …Sie dem Arzt gefühlsmäßig vertrauen können

...der Arzt Ansprechpartner für private Probleme ist (z.B. familiär, beruflich)

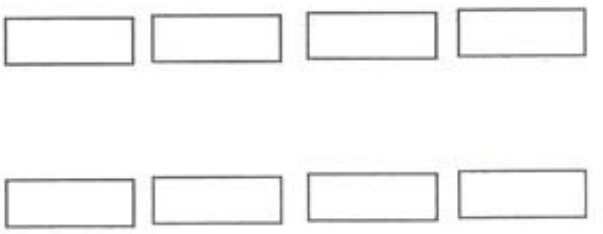
...der Arzt sich sehr gut im Bereich seelischer Störungen auskennt

- ...der Arzt Wert auf medikamentöse Behandlung psychischer Beschwerden legt
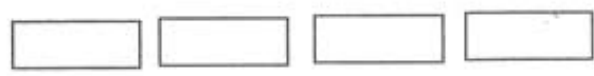

- ...der Arzt auf Selbsthilfegruppen hinweist
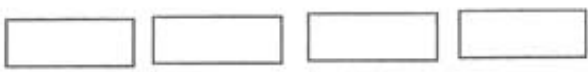

- ...sich der Arzt in Ihre gesundheitliche Lage hineinversetzen kann
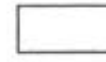
(Fortsetzung)

\section{Ist es Ihnen beim Hausarzt oder Psychiater wichtiger, dass...}

- ...der Arzt seelische und körperliche Beschwerden gleichermaßen ernst nimmt

- ...der Arzt auf Hilfsangebote für Patienten (z.B. finanzielle Hilfen) hinweist

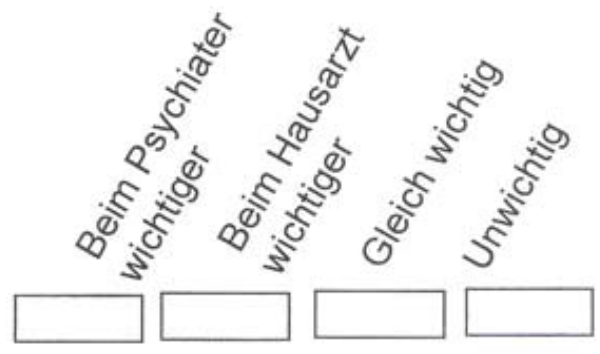

....der Arzt über die Wirkungen und Nebenwirkungen von Psychopharmaka (Begriff ggf. erklären) aufklärt

- ...der Arzt Sie darin unterstützt, Kontakte zu anderen Menschen zu pflegen
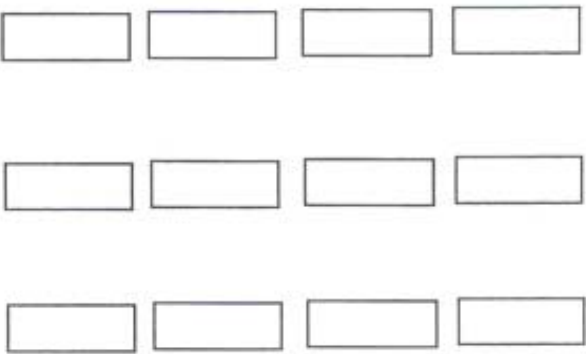

- ...Sie selbst ausführlich Gelegenheit haben, Ihre Geschichte zu erzählen

-...der Arzt mit Ihnen übt, wie Sie mit anderen Menschen über Ihre seelische Erkrankung sprechen können

... der Arzt große Erfahrung in der Behandlung psychischer Störungen gesammelt hat

- ...der Arzt mit Ihnen bespricht, wie Sie Frühwarnzeichen der seelischen Erkrankung erkennen können

- ...der Arzt Sie und Ihre Familie darin unterstützt, harmonisch miteinander auszukommen

- ...der Arzt Maßnahmen zum beruflichen Wiedereinstieg oder Fortkommen anregt

-...Sie den Arzt auch bei Selbstmordgedanken ins Vertrauen ziehen können

....der Arzt Ihnen das Gefühl vermittelt, Sie mit allen Stärken und Schwächen zu akzeptieren

- ...der Arzt frühzeitig auf Zeichen drohender Krankheitsverschlechterung reagiert
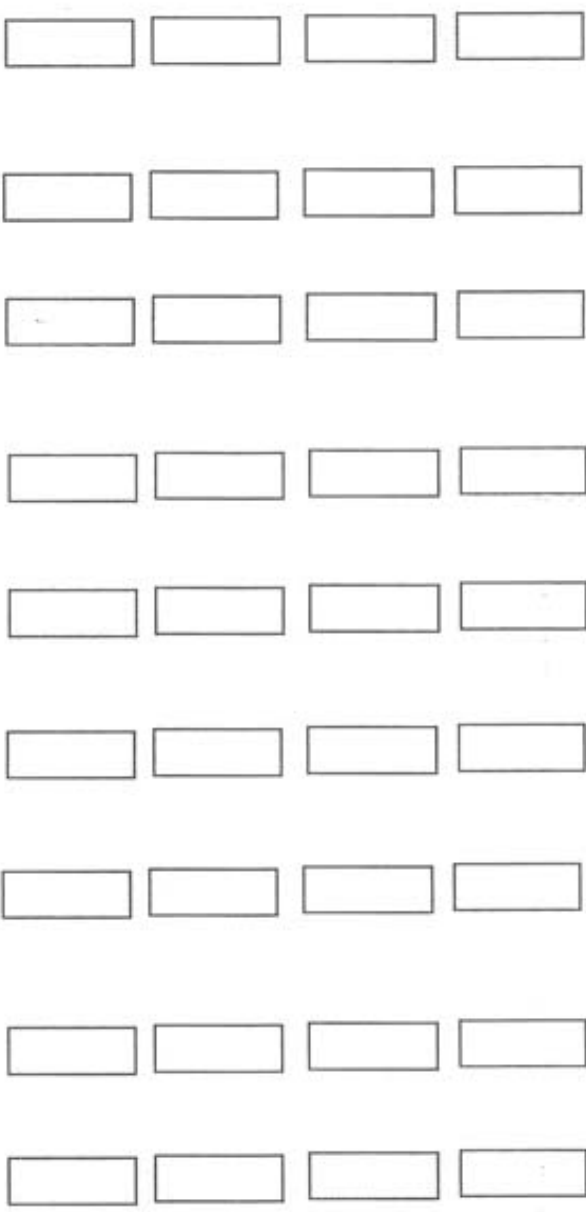

6

...der Arzt psychotherapeutische Gespräche anbietet
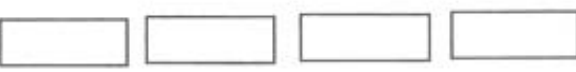

-...der Arzt mit Ihnen darüber spricht, welche Bedeutung die seelische Erkrankung für $\mathrm{Ihr}$ Leben hat
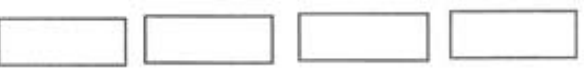


\subsection{Fragebogen Teil 2}

\section{Teil 2}

\section{Bitte bewerten Sie:}

Der Arzt praktiziert in der Nähe Ihres Wohnortes

In der Praxis herrscht eine angenehme Atmosphäre (z.B. keine Hektik)

Sie kennen den Arzt lange

Es ist möglich, schnell einen Termin zu bekommen (innerhalb von 2-3 Tagen)

Der Arzt ist auch außerhalb der üblichen Sprechzeiten erreichbar (z.B. abends, nachts, am Wochenende)

Der Arzt macht Hausbesuche

Der Arzt nimmt sich Zeit für Sie (z.B. auch einmal mehr als $15 \mathrm{~min}$.)

Der Arzt erklärt Ihnen das Ergebnis einer Untersuchung

Der Arzt informiert Sie umfassend über die seelische Erkrankung

Der Arzt bezieht Sie in die Entscheidungen über die medizinische Behandlung mit ein

Der Arzt macht es Ihnen leicht, über belastende Probleme zu sprechen

Der Arzt erklärt Ihnen den Zweck von Untersuchungen / Behandlungen

Der Arzt kann gut zuhören

Der Arzt begegnet Ihnen mit Respekt $\begin{array}{llllllllll}1 & 2 & 3 & 4 & 5 & 6 & 7 & 8 & 9 & 10\end{array}$ trifft nicht zu trifft voll zu

beim Hausarzt beim Psychiater beim Hausarzt beim Psychiater beim Hausarzt beim Psychiater beim Hausarzt beim Psychiater beim Psychiater

beim Hausarzt beim Psychiater

beim Hausarzt beim Psychiater

beim Hausarzt beim Psychiater beim Hausarzt beim Psychiater

beim Hausarzt beim Psychiater

beim Hausarzt beim Psychiater

beim Hausarzt beim Psychiater

beim Hausarzt

beim Psychiater
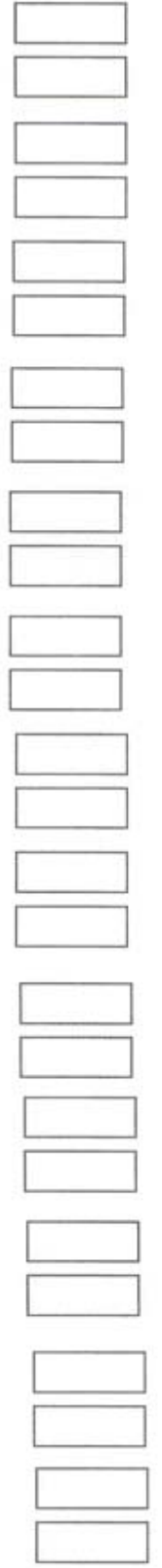

beim Hausarzt beim Psychiater 
(Fortsetzung)

Bitte bewerten Sie:

Der Arzt fragt von sich aus nach seelischen Belastungen (z.B. im Alltag)

Der Arzt nimmt darauf Rücksicht, wie Sie sich die Behandlung Ihrer seelischen Erkrankung vorstellen

Der Arzt achtet auf die vertrauliche Behandlung persönlicher Angaben

Der Arzt ermuntert Sie, Fragen zu stellen

Der Arzt unterstützt und berät Ihre Angehörigen

Der Arzt untersucht Sie sorgfältig körperlich

Der Arzt überweist Sie auch an andere Ärzte

Die Wartezeiten in der Praxis sind kurz (nicht länger als $30 \mathrm{~min}$.)

Der Arzt geht darauf ein, wenn Sie die von ihm verschriebenen Medikamente nicht eingenommen haben

Sie können dem Arzt gefühlsmäßig vertrauen

Der Arzt ist Ansprechpartner für private Probleme (z.B. familiär, beruflich)

Der Arzt kennt sich sehr gut im Bereich seelischer Störungen aus

Der Arzt legt Wert auf medikamentöse Behandlung seelischer Beschwerden

Der Arzt weist auf Selbsthilfegruppen hin

Der Arzt kann sich in Ihre gesundheitliche Lage hineinversetzen
12345678910

trifft nicht zu trifft voll zu beim Hausarzt

beim Psychiater

beim Hausarzt

beim Psychiater

beim Hausarzt

beim Psychiater

beim Hausarzt

beim Psychiater

beim Hausarzt

beim Psychiater

beim Hausarzt

beim Psychiater

beim Hausarzt

beim Psychiater

beim Hausarzt

beim Psychiater

beim Hausarzt

beim Psychiater

beim Hausarzt

beim Psychiater

beim Hausarzt

beim Psychiater

beim Hausarzt

beim Psychiater

beim Hausarzt

beim Psychiater

beim Hausarzt

beim Psychiater

beim Hausarzt

beim Psychiater
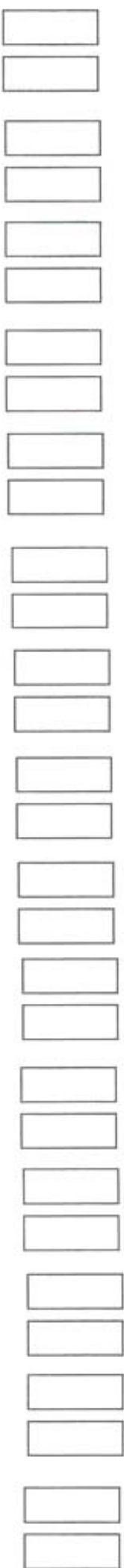
(Fortsetzung)

Bitte bewerten Sie:

Der Arzt seelische und körperliche Beschwerden gleich ernst nimmt

Der Arzt weist auf Hilfsangebote (z.B. finanzielle Hilfen) hin

Der Arzt klärt über die Wirkungen und Nebenwirkungen von Psychopharmaka (Begriff ggf. erklären) auf

Der Arzt unterstützt Sie darin, Kontakte zu anderen Menschen zu pflegen

Sie haben selbst ausführlich Gelegenheit, Ihre Geschichte zu erzählen

Der Arzt übt mit Ihnen, wie Sie mit anderen Menschen über Ihre seelische Krankheit sprechen können

Der Arzt hat große Erfahrung in der Behandlung psychischer Störungen gesammelt

Der Arzt bespricht mit Ihnen, wie Sie Frühwarnzeichen der seelischen Erkrankung erkennen können

Der Arzt unterstützt Sie und Ihre Familie darin, harmonisch miteinander auszukommen

Der Arzt regt Maßnahmen zum beruflichen Wiedereinstieg oder Fortkommen an

Sie können den Arzt auch bei Selbstmordgedanken ins Vertrauen ziehen

Der Arzt vermittelt Ihnen das Gefühl, Sie mit allen Stärken und Schwächen zu akzeptieren

Der Arzt reagiert frühzeitig auf Zeichen drohender Krankheitsverschlechterung

Der Arzt bietet Ihnen psychotherapeutische Gespräche an

Der Arzt spricht mit Ihnen darüber, welche Bedeutung die seelische Erkrankung für Ihr Leben hat $\begin{array}{llllllllll}12 & 2 & 5 & 6 & 7 & 8 & 9 & 10\end{array}$

trifft nicht zu trifft voll zu beim Hausarzt

beim Psychiater

beim Hausarzt

beim Psychiater

beim Hausarzt

beim Psychiater

beim Hausarzt

beim Psychiater

beim Hausarzt

beim Psychiater

beim Hausarzt

beim Psychiater

beim Hausarzt

beim Psychiater

beim Hausarzt

beim Psychiater

beim Hausarzt

beim Psychiater

beim Hausarzt

beim Psychiater

beim Hausarzt

beim Psychiater

beim Hausarzt

beim Psychiater

beim Hausarzt

beim Psychiater

beim Hausarzt

beim Psychiater

beim Hausarzt

beim Psychiater
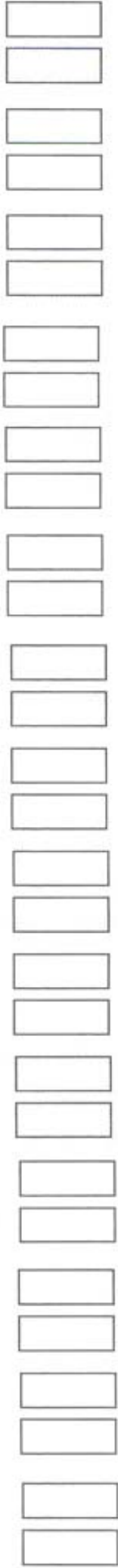


\subsection{Grundauszählung Patienten (Gesamtstichprobe N=111)}

In Klammern finden sich für die einzelnen Fragen Angaben, zur Anzahl der antwortenden Patienten, wenn nicht alle Patienten antworteten.

N (\%)

Muttersprache

- deutsch

- andere Muttersprache, gute deutsche Sprachkenntnisse

- andere Muttersprache, schlechte deutsche Sprachkenntnisse

- sprachliche Verständigung aus anderen Gründen unmöglich

- unbekannt/unklar

Geschlecht

- männlich

- weiblich

Alter (J.)

Minimum:

Maximum:

Mittelwert:

Aktueller Familienstand $(n=110)$

- ledig

- verheiratet/zusammenlebend

- geschieden

- verwitwet

- getrennt lebend

\section{Feste Partnerschaft}

- ja

- nein 
Alter bei erstmaligem Auftreten der psychischen Beschwerden ( $n=107)$ Alter; (J.)

21-30

31-40

$41-50$

$51-60$

Erste psychiatrische Behandlung $(n=107)$

Alter; (J.)

$$
\text { 1-20 }
$$

21-30

31-40

41-50

$51-60$

Anzahl der stationären Aufenthalte $(n=99)$

$0-3$

4-6

40

7-9

3

10-15

9

$>15$

3

Psychische Mitbehandlung in den letzten 12 Monaten

- nein

3

Ja und zwar durch:

- niedergelassene Psychiater/in

- Psychiater/in einer Klinikambulanz

- sonstige

- psychologische Psychotherapeut/in 
Wer ist der Hauptansprechpartner für psychische Probleme?

- niedergelassene Psychiater/in

- Psychiater/in einer Klinikambulanz

- sonstige

- psychologische Psychotherapeut/in

- Hausarzt

Wer ist der Hauptansprechpartner in Fragen der psychopharm.

Medikation?

- niedergelassene Psychiater/in

- Psychiater/in einer Klinikambulanz

- sonstige

- psychologische Psychotherapeut/in

- Hausarzt

Hausarzt bekannt seit (in Jahren)

Minimum:

Maximum:

36

Mittelwert:

Psychiater/Psychologe bekannt seit (in Jahren)

Minimum:

Maximum:

24

Mittelwert: 
Diagnose in eigenen Worten $(n=89)$

- Schizophrenie 39

$\begin{array}{ll}\text { - Psychose } & 27\end{array}$

- Depression 10

- Stoffwechselerkrankung 3

- Neurose

- Endogene Psychose aus dem schizophrenen Formenkreis

- Paranoide Psychose

- Psychische Erkrankung

- Chronische Psychose

- Angstzustände

- Selbstmordgedanken

- Endogene Psychose

- Wahnvorstellungen

\section{Höchster Schulabschluss}

- keinen

- unbekannt

- Hauptschulabschluss

- Mittlere Reife

- Fach-/Hochschulreife

\section{Derzeitige berufliche Situation}

- Vollzeitig berufstätig seit mehr als 6 Monaten

- Teilzeit-berufstätig seit mehr als 6 Monaten 3

- in beschützter Beschäftigung

- Hausfrau/-mann

- in Ausbildung/Studium

- arbeitslos gemeldet

- Erwerbs-/Berufsunfähigkeits-/Früh-/Altersrentner

- anderweitig ohne berufliche Beschäftigung

- beschützte Beschäftigung und Berentung

- unbekannt/unklar 
Wohnsituation

- allein/ in nicht-therapeutischer WG 29

- in eigener Familie/Partnerschaft 32

- bei Eltern (Herkunftsfamilie)

- therapeutische WG oder psychiatrische Familienpflege

- psychiatrische Übergangseinrichtung, Altersheim

- Notunterkunft

- unbekannt

Teilnahme an Selbsthilfegruppe $(n=110)$

- ja

- nein

Größe des Wohnorts ( $n=107)$

- <5.000 Einwohner

- 5.000-25.000 Einwohner

- 25.000-100.000 Einwohner

- >100.000 Einwohner

Besteht eine gesetzliche Betreuung ( $n=106)$

- ja

- nein

\section{Gegenwärtige Medikation}

- ein orales Medikament

- zwei oder mehr orale Medikamente

- Depotmedikation

- Depotmedikation und orale Medikation

- keine Medikation

- unbekannt 
Orale Medikation $(n=107)$

Präparat 1 ( $n=104)$

- Atypische Neuroleptika

- Neuroleptika

- Trizyklische Neuroleptika

- Trizyklische Antidepressiva

- Anticholinergica

- Antidepressiva

- Pfl. Antidepressiva

- Benzodiazepine

- SSRI

- Lithium

Präparat $2(n=56)$ :

- Atypische Neuroleptika

- Neuroleptika

- Trizyklische Neuroleptika

- Trizyklische Antidepressiva

- Anticholinergica

- Antidepressiva

- Pfl. Antidepressiva

- Benzodiazepine

- SSRI

- Lithium

Depotmedikation ( $n=23)$ :

- Neurolepika

- Atypisches Neuroleptika 
Überlegen Sie bitte kurz in welchen Situationen bzw. mit welchem Anliegen Sie sich gefühlsmäßig eher an den Hausarzt

bzw. an den Nervenarzt wenden? (Anm.: Mehrfachantworten waren möglich, Zusammenfassung der freien Antwortmöglichkeiten in aussagekräftigen Kategorien, angegebene Prozentzahlen beziehen sich auf Gesamtstichprobe)

Hausarzt $(n=110)$ :

- nur körperliche Beschwerden

- hauptsächlich seelische Beschwerden

- körperliche/seelische Beschwerden 25

- Überweisung 21

- Blutabnahme

- Medikamente

Nervenarzt ( $n=103)$ :

- Medikamente

- psychische Verschlechterung

- Gespräch

Wieso ist das so? Nennen Sie Gründe dafür?

Motive für den Vorzug des einen Arztes gegenüber dem anderen?

(Anm.: Mehrfachantworten waren möglich, Zusammenfassung der freien

Antwortmöglichkeiten in aussagekräftigen Kategorien, angegebene Prozentzahlen beziehen sich auf Gesamtstichprobe)

Hausarzt ( $n=13)$ :

- fachliche Kompetenz

- Vertrauen

- sonstiges

Nervenarzt ( $n=83)$ :

- fachliche Kompetenz

- Medikamente

- hat sich so ergeben

- sonstiges 
Nennen Sie bitte einige Punkte, die Sie sich besonders an

ihrem Hausarzt wünschen? (Anm.: Mehrfachantworten waren möglich,

Zusammenfassung der freien Antwortmöglichkeiten in aussagekräftigen Kategorien, angegebene Prozentzahlen beziehen sich auf Gesamtstichprobe;

feinere Untergliederung dieser Kategorie finden sich im Text)

Hausarzt $(n=108)$ :

- Persönlichkeit des Arztes

- Fachkompetenz des Arztes

- Vertrauensvolles Verhältnis

- Emotionale Unterstützung

46

- Beratung und Information

- Rahmenbedingungen und Inhalte Konsultation

\section{Nennen Sie einige Punkte, die Ihnen bei Ihrem Nervenarzt}

wichtig erscheinen? (Anm.: Mehrfachantworten waren möglich, Zusammenfassung der freien Antwortmöglichkeiten in aussagekräftigen Kategorien, angegebene Prozentzahlen beziehen sich auf Gesamtstichprobe, feinere Untergliederungen dieser Kategorie finden sich im Text)

Nervenarzt ( $n=109)$ :

- Persönlichkeit des Arztes

- Fachkompetenz des Arztes

- Vertrauensvolles Verhältnis

- Emotionale Unterstützung

- Beratung und Information

- Rahmenbedingungen und Inhalte Konsultation 
Ist es Ihnen beim Hausarzt oder

beim Psychiater wichtiger, dass...

\begin{tabular}{|c|c|c|c|c|}
\hline & $\begin{array}{c}\text { Beim } \\
\text { Psychiater } \\
\text { wichtiger } \\
\text { in } \%\end{array}$ & $\begin{array}{c}\text { Beim } \\
\text { Hausarzt } \\
\text { wichtiger } \\
\text { in } \%\end{array}$ & $\begin{array}{l}\text { Gleich } \\
\text { wichtig } \\
\text { in \% }\end{array}$ & $\begin{array}{l}\text { Unwichtig } \\
\text { in \% }\end{array}$ \\
\hline $\begin{array}{l}\text {...der Arzt in der Nähe Ihres Wohnortes } \\
\text { praktiziert }\end{array}$ & 18 & 16 & 55 & 11 \\
\hline $\begin{array}{l}\text {...in der Praxis eine angenehme Atmo- } \\
\text { sphäre (z.B. keine Hektik) herrscht }\end{array}$ & 28 & 6 & 57 & 9 \\
\hline ...Sie den Arzt lange kennen & 29 & 5 & 53 & 13 \\
\hline $\begin{array}{l}\text {...es möglich ist, schnell einen Termin zu } \\
\text { bekommen (innerhalb von } 2-3 \text { Tagen) }\end{array}$ & 32 & 8 & 50 & 10 \\
\hline $\begin{array}{l}\text {... der Arzt auch außerhalb der üblichen } \\
\text { Sprechzeiten erreichbar ist (z.B. abends, } \\
\text { nachts, am Wochenende) }\end{array}$ & 14 & 18 & 33 & 34 \\
\hline ...der Arzt Hausbesuche macht & 6 & 32 & 24 & 38 \\
\hline $\begin{array}{l}\text {...der Arzt sich Zeit für Sie nimmt (z.B. } \\
\text { auch einmal mehr als } 15 \text { min.) }\end{array}$ & 37 & 4 & 50 & 9 \\
\hline $\begin{array}{l}\text {...der Arzt Ihnen das Ergebnis einer Un- } \\
\text { tersuchung erklärt }\end{array}$ & 8 & 12 & 75 & 5 \\
\hline $\begin{array}{l}\text {...der Arzt Sie umfassend über die seeli- } \\
\text { sche Erkrankung informiert }\end{array}$ & 53 & 1 & 38 & 8 \\
\hline $\begin{array}{l}\text {...der Arzt Sie in die Entscheidungen über } \\
\text { die medizinische Behandlung mit einbe- } \\
\text { zieht }\end{array}$ & 29 & 6 & 50 & 16 \\
\hline $\begin{array}{l}\text {...der Arzt es Ihnen leicht macht, über } \\
\text { belastende Probleme zu sprechen }\end{array}$ & 37 & 5 & 53 & 5 \\
\hline $\begin{array}{l}\text {...der Arzt Ihnen den Zweck von } \\
\text { Untersuchungen / Behandlungen erklärt }\end{array}$ & 13 & 15 & 61 & 12 \\
\hline ...der Arzt gut zuhören kann & 20 & 3 & 77 & 1 \\
\hline ...der Arzt Ihnen mit Respekt begegnet & 95 & 4 & 81 & 6 \\
\hline
\end{tabular}




\begin{tabular}{|c|c|c|c|}
\hline $\begin{array}{l}\text { Beim } \\
\text { Psychiater } \\
\text { wichtiger }\end{array}$ & $\begin{array}{c}\text { Beim } \\
\text { Hausarzt } \\
\text { wichtiger }\end{array}$ & $\begin{array}{c}\text { Gleich } \\
\text { wichtig }\end{array}$ & Unwichtig \\
\hline
\end{tabular}

in $\% \quad$ in $\% \quad$ in $\% \quad$ in $\%$

...der Arzt von sich aus nach seelischen Be-

50

1

32

13

lastungen fragt

...der Arzt darauf Rücksicht nimmt, wie Sie sich die Behandlung Ihrer seelischen Er-

krankung vorstellen

...der Arzt auf die vertrauliche Behandlung persönlicher Angaben achtet

...der Arzt Sie ermuntert, Fragen zu stellen

...der Arzt Ihre Angehörigen unterstützt und berät

...der Arzt Sie sorgfältig körperlich untersucht

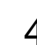

...der Arzt Sie auch an andere Ärzte überweist

...die Wartezeiten in der Praxis kurz sind (nicht länger als $30 \mathrm{~min}$.)

...der Arzt darauf eingeht, wenn Sie die von inm verschriebenen Medikamente nicht eingenommen haben

...Sie dem Arzt gefühlsmäßig vertrauen können

...der Arzt Ansprechpartner für private

Probleme ist (z.B. familiär, beruflich)

...der Arzt sich sehr gut im Bereich seelischer Störungen auskennt

...der Arzt Wert auf medikamentöse

...der Arzt auf Selbsthilfegruppen hinweist

...sich der Arzt in Ihre gesundheitliche Lage hineinversetzen kann schwerden gleichermaßen ernst nimmt 


$\begin{array}{cccc}\text { Beim } & \text { Beim } & \text { Gleich } & \\ \begin{array}{c}\text { Psychiater } \\ \text { wichtiger }\end{array} & \begin{array}{l}\text { Hausarzt } \\ \text { wichtiger }\end{array} & \text { wichtig } & \end{array}$

$\begin{array}{llll}\text { in } \% & \text { in } \% & \text { in } \% & \text { in } \%\end{array}$

...der Arzt auf Hilfsangebote für Patienten

(z.B. finanzielle Hilfen) hinweist

...der Arzt über die Wirkungen und Nebenwirkungen von Psychopharmaka (Begriff ggf. erklären) aufklärt

...der Arzt Sie darin unterstützt, Kontakte zu anderen Menschen zu pflegen

...Sie selbst ausführlich Gelegenheit haben, Ihre Geschichte zu erzählen

...der Arzt mit Ihnen übt, wie Sie mit anderen Menschen über Ihre seelische Erkrankung sprechen können

... der Arzt große Erfahrung in der Behandlung psychischer Störungen gesammelt hat

...der Arzt mit Ihnen bespricht, wie Sie Frühwarnzeichen der seelischen Erkrankung erkennen können

...der Arzt Sie und Ihre Familie darin unterstützt, harmonisch miteinander auszukommen

...der Arzt Maßnahmen zum beruflichen Wiedereinstieg oder Fortkommen anregt $(n=107)$

...Sie den Arzt auch bei Selbstmordgedanken ins Vertrauen ziehen können

...der Arzt Ihnen das Gefühl vermittelt, Sie mit allen Stärken und Schwächen zu akzeptieren

...der Arzt frühzeitig auf Zeichen drohender Krankheitsverschlechterung reagiert

...der Arzt psychotherapeutische Gespräche anbietet

...der Arzt mit Ihnen darüber spricht, welche Bedeutung die seelische
38

43

27

38

47

75

57

29

27

47

26

34

52

59
3

4

2

3

2

2

16

26

16

35

34

23

8

Erkrankung für Ihr Leben hat

Anm.: Basis $\mathrm{N}=111$, einige Fragen wurden z.T. von 1 bis 19 Teilnehmern nicht beantwortet, allerdings wurden 41 der 44 gestellten Fragen von mindestens 105 Teilnehmern beantwortet 
Bitte bewerten Sie:

\begin{tabular}{|c|c|c|c|c|c|c|}
\hline & \multicolumn{3}{|c|}{ Hausarzt } & \multicolumn{3}{|c|}{ Psychiater } \\
\hline & $\begin{array}{l}\text { Trifft } \\
\text { nicht } \\
\text { zu }\end{array}$ & & $\begin{array}{l}\text { Trifft } \\
\text { voll zu }\end{array}$ & $\begin{array}{l}\text { Trifft } \\
\text { nicht } \\
\text { zu }\end{array}$ & & $\begin{array}{c}\text { Trifft } \\
\text { voll zu }\end{array}$ \\
\hline & $1-2$ & $3-8$ & $9-10$ & $1-2$ & $3-8$ & $9-10$ \\
\hline & in $\%$ & in $\%$ & in $\%$ & in $\%$ & in $\%$ & in $\%$ \\
\hline $\begin{array}{l}\text { Der Arzt praktiziert in der Nähe Ih- } \\
\text { res Wohnortes }\end{array}$ & - & 30 & 70 & 5 & 42 & 53 \\
\hline $\begin{array}{l}\text { In der Praxis herrscht eine ange- } \\
\text { nehme Atmosphäre (z.B. keine Hek- } \\
\text { tik) }\end{array}$ & 1 & 44 & 55 & 2 & 31 & 67 \\
\hline Sie kennen den Arzt lange & 5 & 34 & 61 & 4 & 34 & 62 \\
\hline $\begin{array}{l}\text { Es ist möglich, schnell einen Termin } \\
\text { zu bekommen (innerhalb von } 2-3 \\
\text { Tagen) }\end{array}$ & - & 25 & 75 & 3 & 38 & 59 \\
\hline $\begin{array}{l}\text { Der Arzt ist auch außerhalb der üb- } \\
\text { lichen Sprechzeiten erreichbar (z.B. } \\
\text { abends, nachts, am Wochenende) }\end{array}$ & 26 & 44 & 30 & 31 & 44 & 25 \\
\hline Der Arzt macht Hausbesuche & 11 & 23 & 66 & 51 & 21 & 28 \\
\hline $\begin{array}{l}\text { Der Arzt nimmt sich Zeit für Sie } \\
\text { (z.B. auch einmal mehr als } 15 \text { min.) }\end{array}$ & 6 & 46 & 48 & 4 & 37 & 59 \\
\hline $\begin{array}{l}\text { Der Arzt erklärt Ihnen das Ergebnis } \\
\text { einer Untersuchung }\end{array}$ & 5 & 34 & 61 & 7 & 35 & 58 \\
\hline $\begin{array}{l}\text { Der Arzt informiert Sie umfassend } \\
\text { über die seelische Erkrankung }\end{array}$ & 44 & 40 & 17 & 12 & 39 & 49 \\
\hline $\begin{array}{l}\text { Der Arzt bezieht Sie in die Ent- } \\
\text { scheidungen über die medizinische } \\
\text { Behandlung mit ein }\end{array}$ & 18 & 45 & 37 & 20 & 31 & 49 \\
\hline $\begin{array}{l}\text { Der Arzt macht es Ihnen leicht, über } \\
\text { belastende Probleme zu sprechen }\end{array}$ & 8 & 46 & 46 & 3 & 36 & 61 \\
\hline $\begin{array}{l}\text { Der Arzt erklärt Ihnen den Zweck } \\
\text { von Untersuchungen / Behandlun- } \\
\text { gen }\end{array}$ & 8 & 43 & 50 & 8 & 37 & 55 \\
\hline Der Arzt kann gut zuhören & 4 & 32 & 64 & - & 27 & 73 \\
\hline $\begin{array}{l}\text { Der Arzt begegnet Ihnen mit Re- } \\
\text { spekt }\end{array}$ & - & 24 & 76 & 2 & 24 & 74 \\
\hline $\begin{array}{l}\text { Der Arzt fragt von sich aus nach } \\
\text { seelischen Belastungen (z.B. im All- } \\
\text { tag) }\end{array}$ & 26 & 49 & 25 & 10 & 35 & 55 \\
\hline $\begin{array}{l}\text { Der Arzt nimmt darauf Rücksicht, } \\
\text { wie Sie sich die Behandlung Ihrer } \\
\text { seelischen Erkrankung vorstellen }\end{array}$ & 22 & 46 & 32 & 15 & 30 & 55 \\
\hline
\end{tabular}




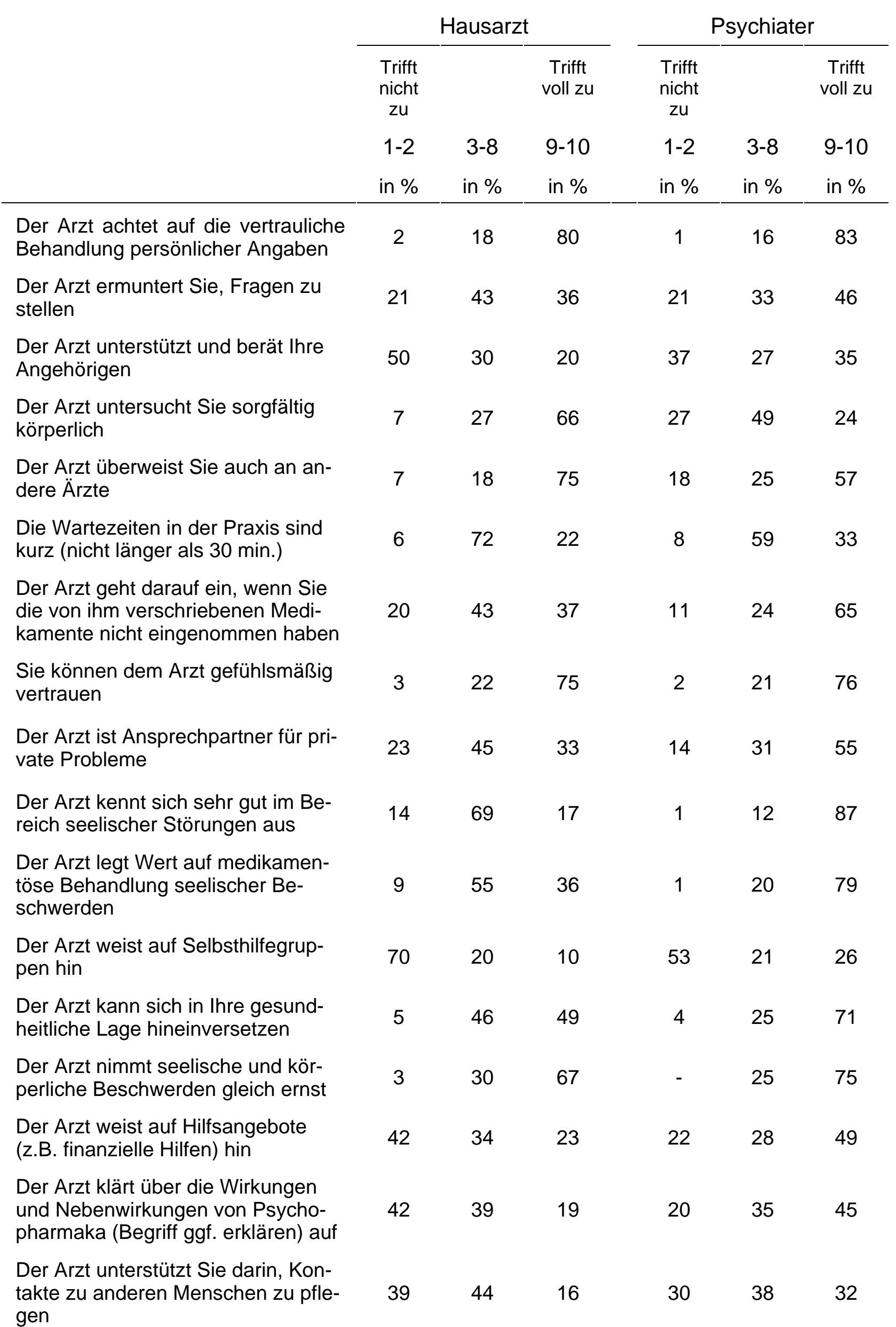


Hausarzt

$\begin{array}{cc}\text { Trifft } & \text { Trifft } \\ \text { nicht } & \text { voll zu }\end{array}$

$\mathrm{zu}$

$\frac{\text { Psychiater }}{\begin{array}{c}\text { Trifft } \\ \text { nicht }\end{array}} \frac{\begin{array}{c}\text { Trifft } \\ \text { voll zu }\end{array}}{\text { zut }}$

$\mathrm{zu}$

\begin{tabular}{cccccc}
$1-2$ & $3-8$ & $9-10$ & $1-2$ & $3-8$ & $9-10$ \\
in $\%$ & in $\%$ & in $\%$ & in \% & in \% & in \% \\
\hline
\end{tabular}

Sie haben selbst ausführlich Gelegenheit, Ihre Geschichte zu erzählen

16

Der Arzt übt mit Ihnen, wie Sie mit anderen Menschen über Ihre seelische Krankheit sprechen können

Der Arzt hat große Erfahrung in der Behandlung psychischer Störungen gesammelt

Der Arzt bespricht mit Ihnen, wie Sie Frühwarnzeichen der seelischen Erkrankung erkennen können

Der Arzt unterstützt Sie und Ihre Familie darin, harmonisch miteinander auszukommen

Der Arzt regt Maßnahmen zum beruflichen Wiedereinstieg oder Fortkommen an

7

71

68

21

1

17

82

51

37

37

12

30

38

Sie können den Arzt auch bei

Selbstmordgedanken ins Vertrauen ziehen

59

29

12

40

24

Der Arzt vermittelt Ihnen das Gefühl, Sie mit allen Stärken und Schwächen zu akzeptieren

Der Arzt reagiert frühzeitig auf Zeichen drohender Krankheitsverschlechterung

$\begin{array}{llllll}4 & 28 & 68 & 2 & 22 & 76\end{array}$

Der Arzt bietet Ihnen psychotherapeutische Gespräche an

Der Arzt spricht mit Ihnen darüber, welche Bedeutung die seelische Erkrankung für Ihr Leben hat

52

35

13

34

Anm.: Die Antworten konnten auf einer 10-stufigen Likert-Skala gegeben werden.

Basis $(N)=111$; einige Fragen wurden z.T. von 1 bis 46 Teilnehmern nicht beantwortet, allerdings wurden 29 der 44 Fragen, die eine Einschätzung des Hausarztes abverlangten, von mind. 100 Teilnehmern beantwortet; ebenfalls mind. 100 Teilnehmer beantworteten 29 der 44 Fragen, die eine Einschätzung des Psychiaters abverlangten. 


\section{Danksagung}

Danken möchte ich allen, die mich in vielfältiger Weise unterstützt haben, dass diese Arbeit beendet werden konnte.

Meinem Doktorvater, Prof. Dr. disc. pol. Wolfgang Himmel, danke ich für die Überlassung des Themas der Doktorarbeit, für wichtige Anregungen und die hervorragende Betreuung der Arbeit.

Außerdem danke ich Frau Dipl. Psych. Anja Rogausch für die kompetente Unterstützung.

Mein besonderer Dank gilt den Ärzten und Arzthelferinnen der beteiligten Praxen und Krankenhäuser sowie dem Mitarbeitern der beteiligten Behindertenwerkstätten, die mich mit großer Freundlichkeit in Ihre Werkstatt eingeladen haben und mir so die Datenerhebung ermöglicht haben.

Schließlich danke ich den Patienten, die sich bereit erklärt haben, an der Studie teilzunehmen. 


\section{Lebenslauf}

Am 09.08.1980 wurde ich als Tochter von Angela und Wolfgang Kühmel in Eberswalde geboren. Ab September 1987 besuchte ich die Polytechnische Oberschule in Eisenach und wechselte dort im August 1991 auf das Ernst-Abbe-Gymnasium, wo ich im Juli 1999 das Abitur ablegte.

Im September 1999 begann ich eine Ausbildung zur Physiotherapeutin in Bad Salzungen, die ich im April 2000 abbrach, um mein Studium für Humanmedizin an der Georg-August-Universität Göttingen aufzunehmen.

In Göttingen legte ich meine ärztliche Vorprüfung 03/2002 ab. Den 1. Teil der ärztlichen Prüfung bestand ich 03/2003, den 2. Teil 8/2005 und den 3. Teil 11/2006.

Mein Praktisches Jahr absolvierte ich im Landesfachkrankenhaus für Psychiatrie in Göttingen, im Albert-Schweitzer-Krankenhaus Northeim und im Kantonsspital Aarau.

Im September 2007 beginne ich meine Assistenzarztausbildung für Psychiatrie und Psychotherapie im Katholischen Krankenhaus Erfurt. 